\title{
The effect of physical and chemical aerosol properties on warm cloud droplet activation
}

\author{
G. McFiggans ${ }^{1}$, P. Artaxo ${ }^{2}$, U. Baltensperger ${ }^{3}$, H. Coe ${ }^{1}$, M. C. Facchini ${ }^{4}$, G. Feingold ${ }^{5}$, S. Fuzzi ${ }^{4}$, M. Gysel ${ }^{1,3}$, \\ A. Laaksonen ${ }^{6}$, U. Lohmann ${ }^{7}$, T. F. Mentel ${ }^{8}$, D. M. Murphy ${ }^{9}$, C. D. O'Dowd ${ }^{10}$, J. R. Snider ${ }^{11}$, and E. Weingartner ${ }^{3}$ \\ ${ }^{1}$ Atmospheric Sciences Group, SEAES, University of Manchester, P.O. Box 88, Manchester, M60 1QD, UK \\ ${ }^{2}$ Instituto de Fisica, Universidade de Sao Paulo, Rua do Matao, Travessa R, 187, CEP 05508-900 Sao Paulo, Brazil \\ ${ }^{3}$ Paul Scherrer Institut, Labor für Atmosphärenchemie, 5232 Villigen PSI, Switzerland \\ ${ }^{4}$ Istituto di Scienze dell' Atmosfera e del Clima, CNR, 40129 Bologna, Italy \\ ${ }^{5}$ NOAA Environmental Technology Laboratory, 325 Broadway, Boulder, Colorado 80305, USA \\ ${ }^{6}$ Department of Applied Physics, University of Kuopio, P.O. Box 1627, 70211 Kuopio, Finland \\ ${ }^{7}$ Institute for Atmospheric and Climate Science, Schafmattstr. 30, ETH Zurich, 8093 Zurich, Switzerland \\ ${ }^{8}$ Forschungszentrum Jülich GmbH, ICG-II: Troposphäre, 52425 Jülich, Germany \\ ${ }^{9}$ NOAA Aeronomy Laboratory, 325 Broadway, Boulder, Colorado 80305, USA \\ ${ }^{10}$ Department of Physics, National University of Ireland, Galway, Ireland \\ ${ }^{11}$ University of Wyoming, Department of Atmospheric Science, Laramie, WY 82071, USA
}

Received: 7 June 2005 - Published in Atmos. Chem. Phys. Discuss.: 12 September 2005

Revised: 13 January 2006 - Accepted: 29 May 2006 - Published: 5 July 2006

\begin{abstract}
The effects of atmospheric aerosol on climate forcing may be very substantial but are quantified poorly at present; in particular, the effects of aerosols on cloud radiative properties, or the "indirect effects" are credited with the greatest range of uncertainty amongst the known causes of radiative forcing. This manuscript explores the effects that the composition and properties of atmospheric aerosol can have on the activation of droplets in warm clouds, so potentially influencing the magnitude of the indirect effect. The effects of size, composition, mixing state and various derived properties are assessed and a range of these properties provided by atmospheric measurements in a variety of locations is briefly reviewed. The suitability of a range of process-level descriptions to capture these aerosol effects is investigated by assessment of their sensitivities to uncertainties in aerosol properties and by their performance in closure studies. The treatment of these effects within global models is reviewed and suggestions for future investigations are made.
\end{abstract}

\section{Introduction}

Aerosol particles affect the radiation balance of the atmosphere in a number of ways. They scatter and absorb in-

Correspondence to: G. McFiggans

(g.mcfiggans@manchester.ac.uk) coming shortwave radiation and absorb outgoing longwave radiation (the "aerosol direct effect" McCormick and Ludwig, 1967; Charlson and Pilat, 1969; Haywood and Boucher, 2000, Charlson et al., 1992). Aerosol particles that act as cloud condensation nuclei cause changes in droplet number affecting the albedo and persistence of clouds; these are respectively termed the "Twomey (first) and the cloud lifetime (second) aerosol indirect effects" (Warner, 1968; Twomey, 1974; Albrecht, 1989; Liou and Ou, 1989; Lohmann and Feichter, 2005). The Twomey effect refers to the aerosolinduced increase in cloud number droplet for a constant liquid water content whereas the cloud lifetime effect is a result of the reduced precipitation efficiency of the more numereous smaller cloud droplets. Absorbing aerosol has also been shown to cause local warming of the atmosphere, which may result in stabilisation of the sub-cloud layer, and large-scale burn-off of clouds. This has been termed the "semi-direct effect" (Fischer and Grassl, 1975; Hansen et al., 1997; Ackerman et al., 2000; Johnson et al., 2004).

The aerosol indirect effect is currently credited with the greatest range of uncertainty amongst the known causes of radiative forcing (Ramaswamy et al., 2001); this range is stated as being around 4 times the uncertainty associated with forcing by radiatively active gases. That its absolute magnitude may be comparable to that from radiatively active gases necessitates greatly improved quantification of the factors affecting and contributing to the aerosol indirect effect.

Published by Copernicus GmbH on behalf of the European Geosciences Union. 
The primary tool at our disposal for assessing aerosol effects on clouds and future climate states is the general circulation model (GCM). These models are required to describe a large number of coupled processes which, because of the enormous range of spatial and temporal scales that need to be addressed, and the associated computational burden, must to a large extent be simplified (e.g. Lohmann and Feichter, 2005). The difficulty in meaningfully incorporating a realistic description of aerosol-cloud interactions into GCMs should not be underestimated. Large scale models have difficulty in representing convection and clouds, which are typically parameterised as subgrid processes. Therefore, their ability to represent the macroscale features of clouds (spatial coverage, depth, hydrometeor content) is itself a challenge. In addition, they cannot represent the magnitude of the updraught velocities which may have an important bearing on the local microphysical cloud properties (droplet number, effective radius etc.). The cloud-scale updraught velocity relevant for cloud droplet activation is usually approximated, either by assuming a Gaussian distribution or relating subgridscale fluctuations to the turbulent kinetic energy (Ghan et al., 1997; Lohmann et al., 1999). It is not the intent of this article to investigate the broad question of dynamical cloud systems, their effect on radiation and the incorporation of these, and other coupled effects into large-scale models. Neglect of these problems is not a reflection of their relative importance in cloud radiative forcing. The main purpose of this review is to identify one particular and important aspect of the aerosol indirect effect, namely, the properties of aerosol which dominate their activation in warm clouds. This aspect of the indirect effect is accessible to investigation by observations alone. For example, the relationships between droplet number $\left(N_{d}\right)$ and aerosol number $\left(N_{a}\right)$ and between effective droplet radius $\left(r_{\text {eff }}\right)$ and $N_{a}$ may both be directly probed. Should all dynamical considerations remain relatively constant, such observations can be used to derive relationships between the aerosol distribution and cloud distribution properties.

This article will review the currently-available observational evidence for the compositional complexity of atmospheric aerosol and the derived properties of their size and composition distributions that are thought to affect their ability to act as cloud condensation nuclei $(\mathrm{CCN})$. An increasing body of evidence suggests that the complexity of atmospheric aerosol may preclude realistic treatment of cloud formation using the levels of simplification incorporated into all large-scale models (and even most process-level descriptions). The frequent assumptions of an externally mixed inorganic salt aerosol appear not to be applicable even in the most pristine regions. Even this description is more complex than that employed in many climate models (Wilson et al., 2001; Gong and Barrie, 2003). Long-range transport of polluted plumes lofted into the free troposphere followed by sporadic re-entrainment (Clarke et al., 1999) ensures that remote regions are not always free from anthropogenic in- fluence (certainly in the Northern Hemisphere) - and cloud formation in these regions may be particularly sensitive to such input. In addition, the variety of natural primary and secondary sources can also lead to a complexity, not broadly appreciated until recent years largely resulting from the numerous organic species contributing to the aerosol burden (Kanakidou et al., 2005). More obviously polluted air such as that originating from densely-populated continental regions (or regions otherwise influenced by human activity, e.g. biomass burning) are frequently heavily laden with an external mixture of internally-mixed multicomponent aerosol distributions (see Sect. 3.2.3).

It might be expected that cloud droplets forming in rising air parcels containing such burdens of multicomponent aerosol may not behave in the same way as those formed by the activation of simple salt particles. A range of techniques have been used to describe how aerosol particles behave as relative humidity approaches and then exceeds saturation. A summary of the fundamental theoretical approaches used to investigate droplet activation is presented, ranging from relatively well-established conventional application of Köhler theory to recently developed theoretical and laboratory-based extensions reflecting the increased aerosol complexity (Shulman et al., 1996; Kulmala et al., 1997). The effects of such extensions are discussed and the relative importance under a range of conditions assessed (Sect. 4).

There have been recent attempts to reconcile field observations of aerosol composition with those of derived properties related to cloud activation. Such studies may be broadly categorised as "hygroscopic growth closure", "CCN closure" and "droplet number closure" investigations. The difficulties associated with each level of closure are different and the droplet number closure is significantly more difficult. An appraisal of these difficulties and the current status of progress in such studies are reviewed in Sect. 5.

This paper therefore aims to examine the state-of-thescience to establish i) the dominant characteristics of the atmospheric aerosol (in so far as they may influence cloud activation) ii) which aerosol properties should be most accurately captured to investigate cloud formation iii) how these properties can be represented at a detailed process level iv) whether current representations of these properties in larger scale models can adequately capture the important behaviour of the aerosol and v) how improved representations may be developed to investigate aerosol effects on cloud formation. Suggestions of which properties should be captured to enable accurate representation of aerosol effects on cloud formation follow from this examination.

A final note on the scope of this article: whilst the effects of atmospheric aerosol properties on warm cloud activation are subject to significant uncertainties, those surrounding the roles of aerosol particles as ice nuclei (IN) are much greater still. The article will therefore be limited to warm clouds and will not attempt to consider mixed-phase or cold clouds. 


\section{Theory of activation of aerosol particles in warm clouds}

The description of the equilibrium size of a droplet with water saturation ratio, founded on the early work of Köhler (1936), is now well-established and can be readily derived from the Clausius-Clapeyron equation modified to give a general equilibrium relation between an aqueous salt solution droplet and water vapour:

$$
\frac{e}{e_{s}}=a_{w} \exp K_{e}=a_{w} \exp \left(\frac{2 v_{w} \sigma_{s o l / v}}{R T r}\right)
$$

where

$e$ is the vapour pressure of water,

$e_{s}$ is the saturation vapour pressure of water,

$\left(e / e_{s}=S\right.$, is known as the saturation ratio),

$a_{w}$ is the water activity,

$K_{e}$ is the Kelvin factor,

$v_{w}$ is the partial molar volume of water,

$\sigma_{\mathrm{sol} / \mathrm{v}}$ is the surface tension of the solution at the composition of the droplet,

$R$ is the universal gas constant,

$T$ is the droplet temperature,

$r$ is the particle radius.

This form of the Köhler equation is not generally accessible to analytical solution and a number of texts (Rogers and Yau, 1989; Pruppacher and Klett, 1997; Chýlek and Wong, 1998; Seinfeld and Pandis, 1998) provide standard derivations to yield the simplified form of the Köhler equation:

$S=\frac{e}{e_{s}} \approx 1+\frac{A}{r}-\frac{B}{r^{3}}$

where $A=\frac{2 M_{w} \sigma_{w / v}}{R T \rho_{w}}$ and $B=\frac{v m_{s} M_{w}}{M_{s}\left(4 / 3 \pi \rho_{w}\right)}$, where $v$ is the number of dissociated ions per solute molecule, $m_{s}$ is the the solute mass and subscripts $s$ and $w$ relate to solute and water properties, respectively. The term in $A$ is denoted the Kelvin or curvature term, and that in $B$, the Raoult or solute term.

This latter form of the equation assumes that the droplet behaves ideally, i.e. that the practical osmotic coefficient of the salt, $\phi=1$, where

$a_{w}=\exp \left(-\frac{v n_{s}}{n_{w}} \phi_{s}\right)$

and that the number of ions in solution is independent of solution concentration. Equation (2) further assumes that the solute is completely soluble and it implicitly follows that the solution droplet is assumed homogeneous - that the composition is independent of distance from droplet centre. It is further assumed that the surface tension and density of the growing droplet are equal to those of water. In addition to the assumptions relating to physico-chemical properties of the activating particles which may be more or less accurate depending on the supersaturation, there are numerical approximations such as that associated with the Taylor series expansion of the exponential which limit the range of applicability of Eq. (2). Figure 1 shows the contribution of the Kelvin and Raoult terms to the activation behaviour of a $200 \mathrm{~nm}$ dry diameter ammonium sulphate particle.

This form of the expression shows a single characteristic maximum in supersaturation for a given dry composition and size, known as the critical supersaturation, $S_{c}$, associated with a unique size, denoted the critical radius, $r_{c}$ or diameter, $D_{c}$. Using the simplified expression (2), the analytical solutions for the critical quantities are:

$r_{c}=\left(\frac{3 B}{A}\right)^{1 / 2}$

$S_{c}=\left(\frac{4 A^{3}}{27 B}\right)^{1 / 2}$

For an increasing environmental value of $S$ below $S_{c}$ there is a unique equilibrium droplet size. Once the droplet grows beyond its critical size (i.e. as the environmental $S$ increases above $S_{c}$ ) the droplet will exhibit unimpeded growth unless the environmental $S$ reduces below the equilibrium value of $S$ at the instantaneous value of $r$. In this case, with no further change in $S$, the droplet will evaporate to its sub-critical equilibrium size.

The Köhler expression can be envisaged as the competition between the two expressions of component properties determining activation of particles; the curvature term and the solute term. The solute terms depends first on the number of solution molecules and then on the dissociation of these molecules. The effect can be illustrated for two frequently assumed cloud condensation nuclei types: ammonium sulphate and sodium chloride. $\left(\mathrm{NH}_{4}\right)_{2} \mathrm{SO}_{4}$ has a molecular weight of $132 \mathrm{gMol}^{-1}$ while that of $\mathrm{NaCl}$ is $58.5 \mathrm{gMol}^{-1}$. Thus, in the absence of dissociation, a given mass of $\mathrm{NaCl}$ in solution would yield 2.26 times more dissolved molecules than $\left(\mathrm{NH}_{4}\right)_{2} \mathrm{SO}_{4}$. Assuming full dissociation (infinite dilution), $\left(\mathrm{NH}_{4}\right)_{2} \mathrm{SO}_{4}$ yields 3 ions while $\mathrm{NaCl}$ yields 2 , so the net effect of the molecular mass and dissociation is that $\mathrm{NaCl}$ is $2.26 / 1.5=1.5$ times more active than $\left(\mathrm{NH}_{4}\right)_{2} \mathrm{SO}_{4}$ for the same dry mass of particle (the $S_{c}$ ratio is around 1.22 due to the square root dependence). This is illustrated in Fig. 2 where the peak supersaturation is plotted versus dry diameter for particles comprising each electrolyte. This figure directly illustrates the significant differences in the critical supersaturation as a function of both the chemical composition and dry size of a particle (Raoult and Kelvin effects).

The atmospheric aerosol does not solely comprise suspended completely soluble inorganic salt solution particles. A modification to the Raoult term was reported by Hänel 


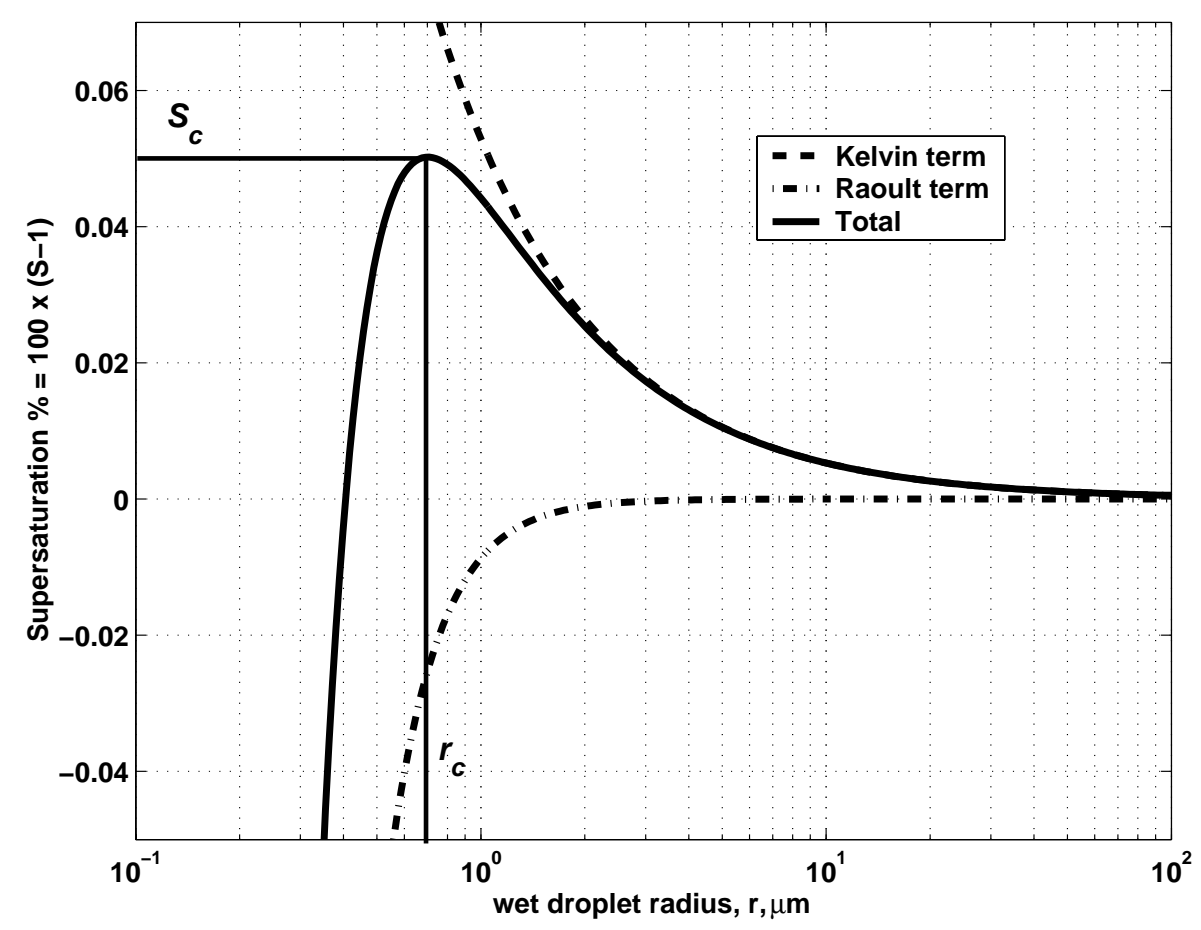

Fig. 1. The Köhler equation can be envisaged as the competition between the curvature (Kelvin) and solute (Raoult) terms.

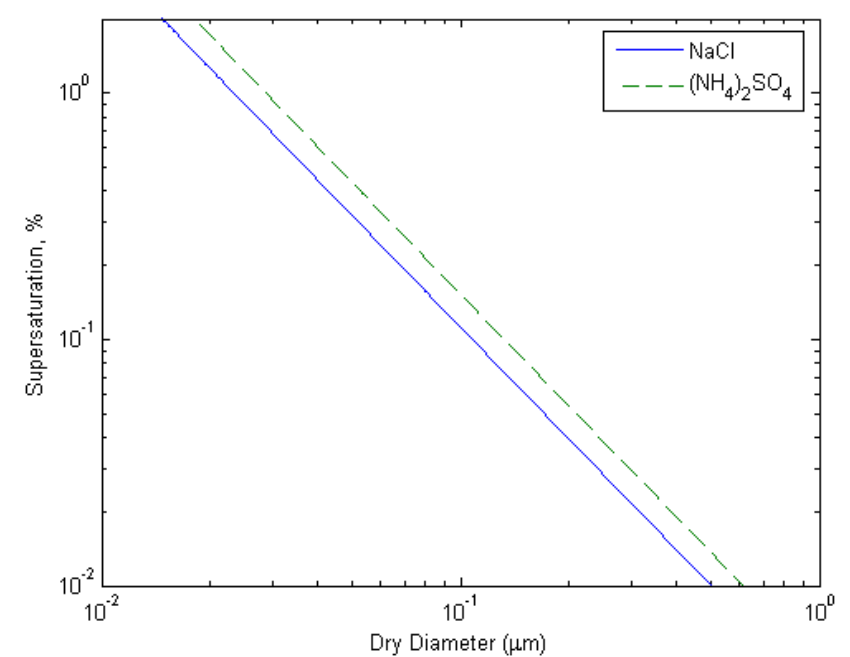

Fig. 2. Critical supersaturation as a function of dry size for $\mathrm{NaCl}$ and $\left(\mathrm{NH}_{4}\right)_{2} \mathrm{SO}_{4}$ particles.

(1976) to allow explicit consideration of internally mixed completely insoluble inclusions:

$B=\frac{\varepsilon v m_{s} M_{w}}{M_{s}\left(4 / 3 \pi \rho_{w}\right)}$

where $\varepsilon$ is the soluble mass fraction of the dry particle.

Figure 3 shows the form of activation curves for a range of particles of varying dry diameter and initial soluble mass fractions. A discussion of more rigorous treatments of limited component solubility is presented in Sect. 4.1.

\section{The composition and properties of atmospheric aerosol relevant to the indirect effect}

Suspended particulate material in the atmosphere is highly variable. Atmospheric aerosol particles cover four or five decades in size from a few nanometers to several tens or even hundreds of microns and the loading and composition are extremely source and location dependent. Studies of cloud formation invariably rely on a simplified model of input aerosol composition distributions. The specific description is dependent on the particular atmospheric scenario. This section presents an overview of the characteristics of atmospheric aerosol as they relate to cloud droplet activation.

\subsection{Characteristic size and composition of atmospheric aerosol}

\subsubsection{Size distributions of ambient aerosol}

It can be seen from Figs. 2 and 3 that the activation of aerosol particles is strongly dependent on the dry size. For any given composition and supersaturation (which, around cloud base, is directly proportional to updraught velocity), whether a particle activates is solely dependent on its dry size. At constant updraught velocity, a distribution of such particles of varying sizes will activate if their corresponding critical radius is 


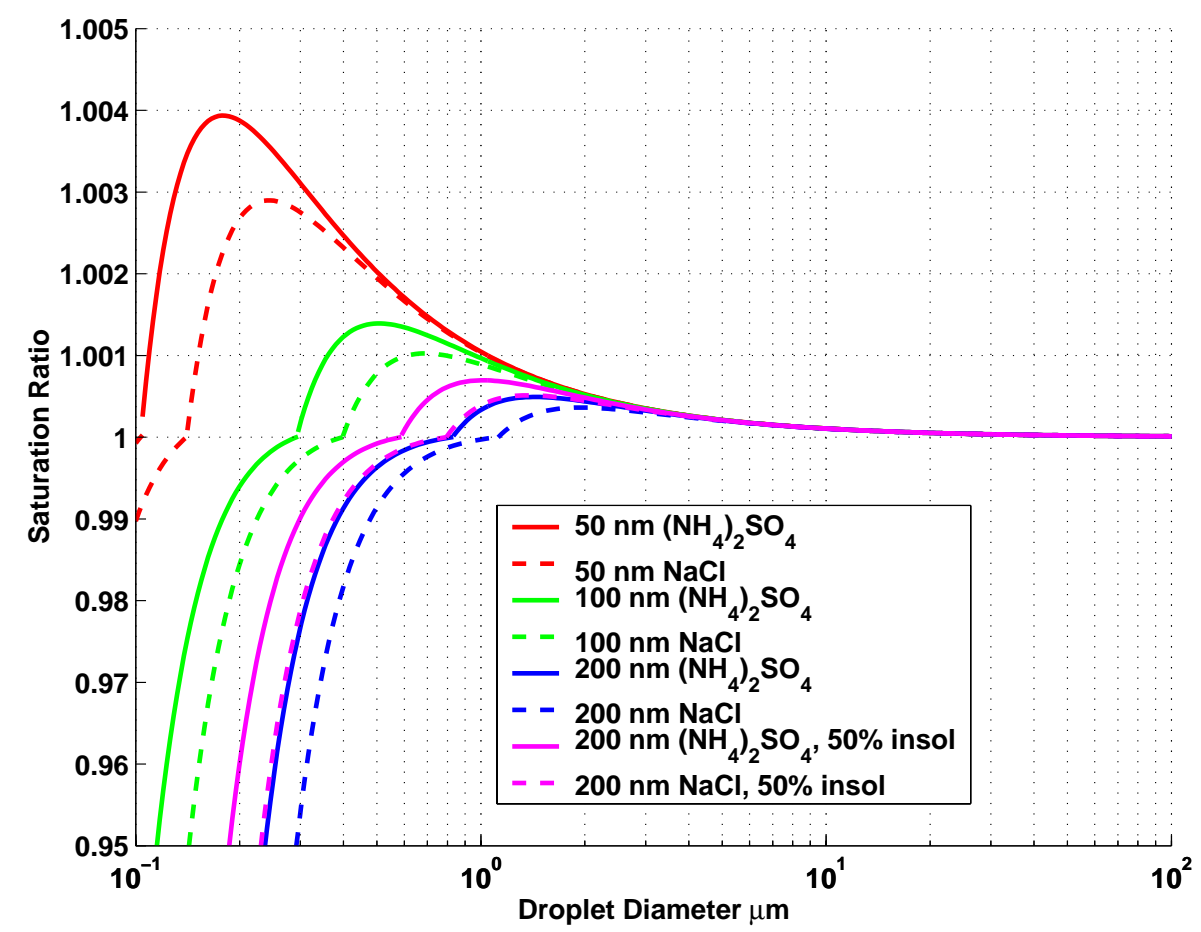

Fig. 3. Activation curves for a range of dry diameter of salt $\left(\left(\mathrm{NH}_{4}\right)_{2} \mathrm{SO}_{4}-\right.$ solid, $\mathrm{NaCl}-$ dashed) particles (red, green and blue curves) and for $200 \mathrm{~nm}$ particles containing $50 \%$ by mass insoluble core (magenta).

reached. As more particles activate and grow, they will compete for available water vapour. The water supersaturation will continue to rise above cloud base but will slow as growing droplets scavenge the water vapour and relatively fewer additional (smaller) aerosol particles will activate. When supersaturation sources and sinks balance, the peak supersaturation is reached - usually within a few 10's of metres above cloud base. Following this point, the growing droplet population will lead to a reduction in supersaturation. No new particles will activate and the most recently activated droplets may evaporate. Some particles will not have sufficient time to reach their critical radius even though their critical supersaturation is reached. This results from water vapour scavenging by the larger droplets reducing supersaturation to below the critical value of the smaller particles before sufficient water vapour can condense (such kinetic limitations are discussed further in Sect. 4). Only particles reaching a certain size will survive and grow. Some of the largest particles may not actually activate, but may be large enough to be considered as droplets since even at their subcritical sizes they will often be greater than 10 or 20 microns in radius, deplete water vapour, and even act as collector drops. A pseudo "steady-state" or quasi-equilibrium is eventually reached for a constant updraught velocity where the decrease in saturation ratio by condensation to the droplet population and the increase in saturation ratio owing to the updraught maintains a broadly constant supersaturation with increasing altitude with the maximum just above cloud base. Numerous implementations of simple adiabatic cloud parcel models exist which describe heat transfer and mass transfer of water vapour between an adiabatically cooling air parcel and the aerosol/droplet population based on fundamental thermodynamic principles (see Howell, 1949; Mordy, 1959; Pruppacher and Klett, 1997; Seinfeld and Pandis, 1998).

Figure 4 demonstrates the predicted behaviour of an idealised lognormal $\left(\mathrm{NH}_{4}\right)_{2} \mathrm{SO}_{4}$ aerosol population with height above cloud base at an updraught velocity of $0.5 \mathrm{~ms}^{-1}$ using such a model. It clearly shows how the droplets activated from the larger classes of aerosol continue to grow above the supersaturation maximum at the expense of the smaller classes of activated particle which evaporate to below their critical radius. The model uses the form of the Köhler equation shown in Eq. (1).

Given this behaviour, it can be seen that both the number of particles in a given size range and the gradient of the distribution in certain critical size ranges will determine its activation behaviour moving into supersaturation. The Twomey (1959) analytical solution to this problem:

$$
N=c\left(100+S^{*}\right)^{k} \text { and } S^{*}=\left(\frac{A(T, P) w^{3 / 2}}{c k \beta(3 / 2, k / 2)}\right)^{1 /(k+2)}
$$

where $c$ is proportional to the $\mathrm{CCN}$ concentration at $1 \%$ supersaturation, $w$ is the updraught velocity, $k$ is the slope parameter of the CCN size spectrum and $N$ is the number 


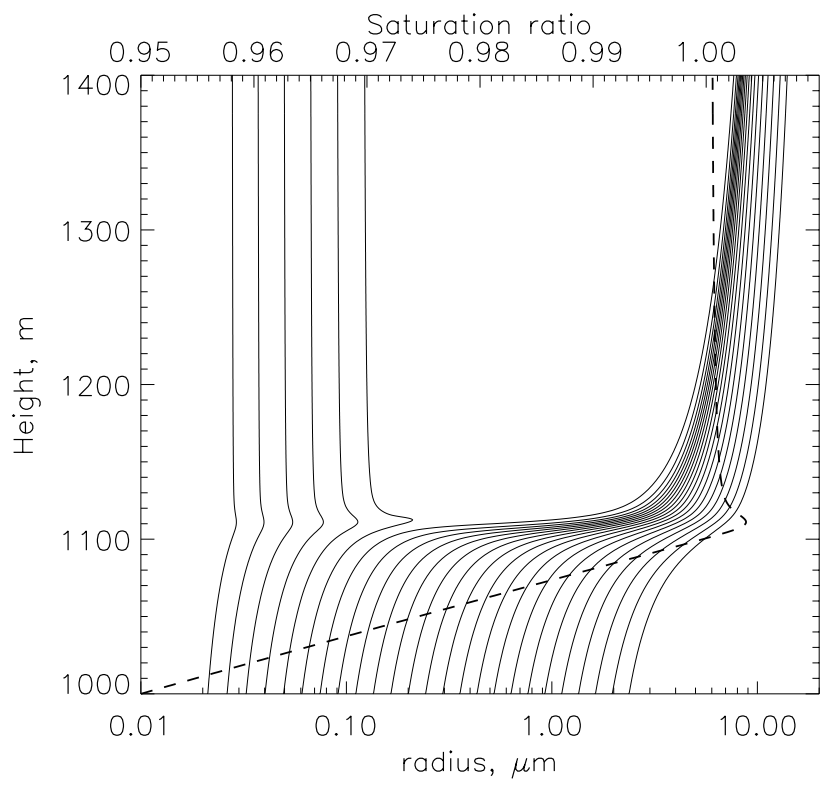

Fig. 4. Simulation showing the change in droplet radius with height in a simulation initialised with an ammonium sulphate aerosol with a geometric mean diameter of $140 \mathrm{~nm}$, a geometric standard deviation, $\sigma_{g}$ of 1.7 and aerosol number concentration of $300 \mathrm{~cm}^{-3}$ (corresponding to a total mass loading of $0.76 \mu \mathrm{gm}^{-3}$ ). The simulation was started at an $R H$ of $95 \%$ at $1000 \mathrm{~m}$. Solid lines represent selected aerosol size classes. The dashed line is the saturation ratio.

of nuclei activated at peak supersaturation $S^{*}$, indicates the relative sensitivity of activated drop number to aerosol size distribution parameters. $A(T, P)$ is a function of temperature $T$ and pressure $P$ and $\beta$ is the complete beta function. This expression works well for modest values of $k$ and was evaluated for southern-hemisphere cumulus clouds. Although the analysis assumes a Junge power-law distribution, the essential features of sensitivity to size parameters and updraught is evident. For example, drop activation is increasingly more sensitive to updraught velocity when size distributions have larger values of $k$ (steeper decreases in concentration with increasing size). Conversely, at smaller values of $k, N$ is determined primarily by $c$ (Twomey, 1977). Jaenicke (1988) pointed out that the power-law representation of the CCN spectrum may not be realistic, particularly for a multi-component aerosol population. Theoretical and model-based analyses of activation in terms of lognormal size distributions have been reported by Cohard et al. (1998); Feingold (2001, 2003); Rissman et al. (2004); Abdul-Razzak and Ghan (2000); Nenes and Seinfeld (2003); Fountoukis and Nenes (2005); and experimentally determined (e.g. Martinsson et al., 1999).

The updraught velocity can range from around $0.1 \mathrm{~ms}^{-1}$ in stratiform clouds to in excess of $15 \mathrm{~ms}^{-1}$ in deep convective or orographically-forced clouds. The supersaturations associated with such a range in updraught velocity mean that fully soluble particles of greater than $\sim 40 \mathrm{~nm}$ diameter may activate under certain atmospheric circumstances. Studies of low clouds have found minimum activated diameters ranging from 40 to $140 \mathrm{~nm}$ (Hoppel et al., 1996; Leaitch et al., 1996; Flynn et al., 2000; Komppula et al., 2005). Smaller particles might be activated in the strong updrafts of vigorous convection, especially in unpolluted environments. The activation of particles above this dry size threshold is a strong function of both the supersaturation and the form of the size and composition distribution. A primary limitation of the approach of Twomey is that it does not account for the multi-component size and composition of the $\mathrm{CCN}$ population.

3.1.2 A practical illustration of the dynamic competition effects of a simple multi-component aerosol population

The Twomey equation, and most others developed to link number of activated droplets to a sub-cloud aerosol concentration (either mass or number), usually assume that the aerosol type is relatively homogeneous. Some parameterisations yield a monotonic increase in cloud droplet concentration (e.g., Boucher and Lohmann, 1995) whereas others show a tendency to saturate (for example Hegg, 1984; Jones et al., 1994). In general they are well approximated by powerlaw dependence of $N_{d}$ on $N_{a}$ or the mass concentration of aerosols. The parameterisations of Ghan et al. (1998) and Feingold (2001), exhibit similar behaviour but, following saturation, show a decrease in $N_{d}$ with further increases with $N_{a}$ at very high aerosol concentrations (order $10000 \mathrm{~cm}^{-3}$ ).

Such assumptions may hold for a single component aerosol but not for an aerosol of significantly different multiple components. The breakdown of these assumptions was well illustrated by Ghan et al. (1998) and O'Dowd et al. (1999) for typical maritime cloud conditions.

O'Dowd et al. (1999) conducted parcel model simulations of the impact of increasing $\mathrm{CCN}$ concentrations on cloud droplet concentration using typical marine sulphate and seasalt size distributions and found that an increase in cloud droplet concentration did not necessarily follow as the $\mathrm{CCN}$ population increases. The aerosol properties were described by three modes; one sulphate mode with a modal diameter of $150 \mathrm{~nm}$ and a geometric standard deviation, $\sigma_{g}$ of 1.4 ; a film drop sea-salt mode with modal diameter of $200 \mathrm{~nm}$ and $\sigma_{g}$ of 1.9 and a super micron mode of $2 \mu \mathrm{m}$ and $\sigma_{g}$ of 2 . The amplitude of each mode was varied within the frequently observed constraints that the amplitude of the sulphate mode is typically greater than those of the sea-salt modes and the amplitude of the film drop mode is always significantly greater than that of the jet drop mode. Simulations were conducted for scenarios where the sulphate aerosol concentration was varied under a range of fixed sea-salt conditions. Sea-salt $\mathrm{CCN}$ were added to the population with the base case of $3 \mathrm{~cm}^{-3}$, increasing to a maximum of $57 \mathrm{~cm}^{-3}$ sea-salt particles. 


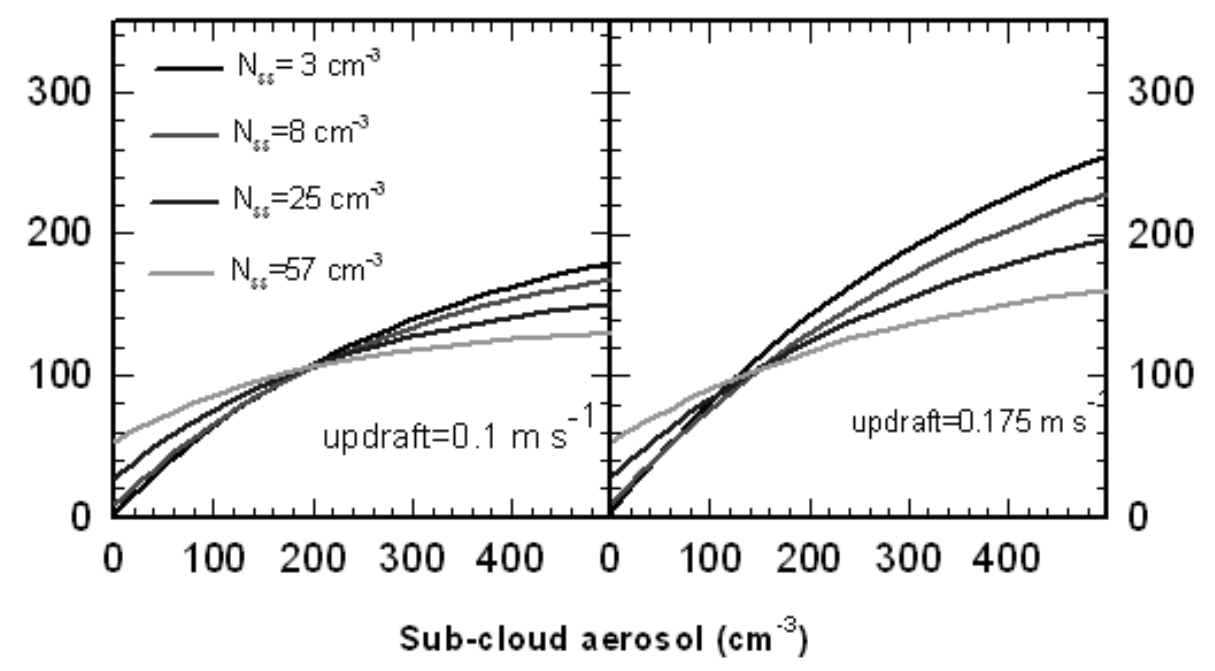

Fig. 5. Cloud droplet concentration as a function of sub-cloud aerosol where the sub-cloud aerosol comprises an external mix of sulphate and sea-salt CCN.

The simulations results are shown in Fig. 5 for updraughts of $0.1 \mathrm{~ms}^{-1}$ and $0.175 \mathrm{~ms}^{-1}$. For low sulphate CCN concentrations, the addition of sea-salt $\mathrm{CCN}$ increases the number of activated droplets significantly while for high sulphate concentrations, the number of activated droplets decreases significantly. For the higher updraught, the point at which the result of the addition of sea-salt nuclei switches from an increase to a decrease in droplet concentration reduces for the higher updraught and the impact of the reduction in droplet concentration increases for increasing updraught.

The main processes driving this phenomenon are (1) seasalt $\mathrm{CCN}$ are typically larger than sulphate $\mathrm{CCN}$; (2) for a given size, sea-salt is more active as a $\mathrm{CCN}$ than sulphate; (3) although the relative concentration of larger sea-salt CCN is significantly lower than sulphate $\mathrm{CCN}$, they contribute to a significant reduction in the peak supersaturation reached in cloud and thus inhibit the activation of sulphate nuclei. This example demonstrates that for even simple two-component aerosol systems the dynamic competition is quite complex and non-linear and that the effect of increasing the availability of CCN does not necessarily lead to an increase in cloud droplet concentration. Similar non-linearities are evident in the effects of composition on droplet activation and caution should be exercised in translating a composition change to an equivalent change in drop number concentration (Ervens et al., 2005). The results of such responses are strongly dependent on water vapour supply (i.e. updraught) and condensation rates (dependent on size distribution and composition).

The activation of seasalt and sulphate in marine stratiform cloud described in this section is a particularly simple case in terms of both composition and the limited range of updraught velocity. Ambient aerosol size distributions are highly variable from location to location. The reader is referred to a range of review articles for a broad and detailed characteri- sation of these properties (e.g. Whitby, 1978; Van Dingenen et al., 2004).

Figure 6 shows representative average distributions in a variety of locations. Most particles greater than $200 \mathrm{~nm}$ diameter with moderate amounts of soluble material will activate under reasonable supersaturations. Assuming that those particles greater than $200 \mathrm{~nm}$ in Fig. 6 are moderately soluble, it can be seen that the sizes critical to determining the droplet number in an aerosol population fall in the range with significant contributions from both Aitken and accumulation mode particles (around $100 \mathrm{~nm}$ diameter). It is therefore necessary to capture the features of the aerosol size distribution in both modes in order to realistically describe cloud activation behaviour, (Martinsson et al., 1999). The following sections investigate further properties of real atmospheric aerosol and the potential impacts of these properties on cloud activation.

It should be noted that, the critical size range for cloud activation of about 50 to $150 \mathrm{~nm}$ is not accessible to most optical sizing instruments, but may be probed by mobility instruments. This significantly reduces the amount of data available at cloud altitudes because mobility analyses can be challenging on aircraft due to the time required to scan a size distribution.

\subsubsection{Relative importance of size distribution, composition and updraught}

Feingold (2003) performed a sensitivity analysis comparing aspects of the relative importance of aerosol size and composition, in so far as both properties affect activation, using a cloud parcel model. Input aerosol size distributions (parameterised as lognormal functions described by $N_{a}, r_{g}, \sigma_{g}$ ), and prescribed updraught velocities, $w$, were varied over a large 

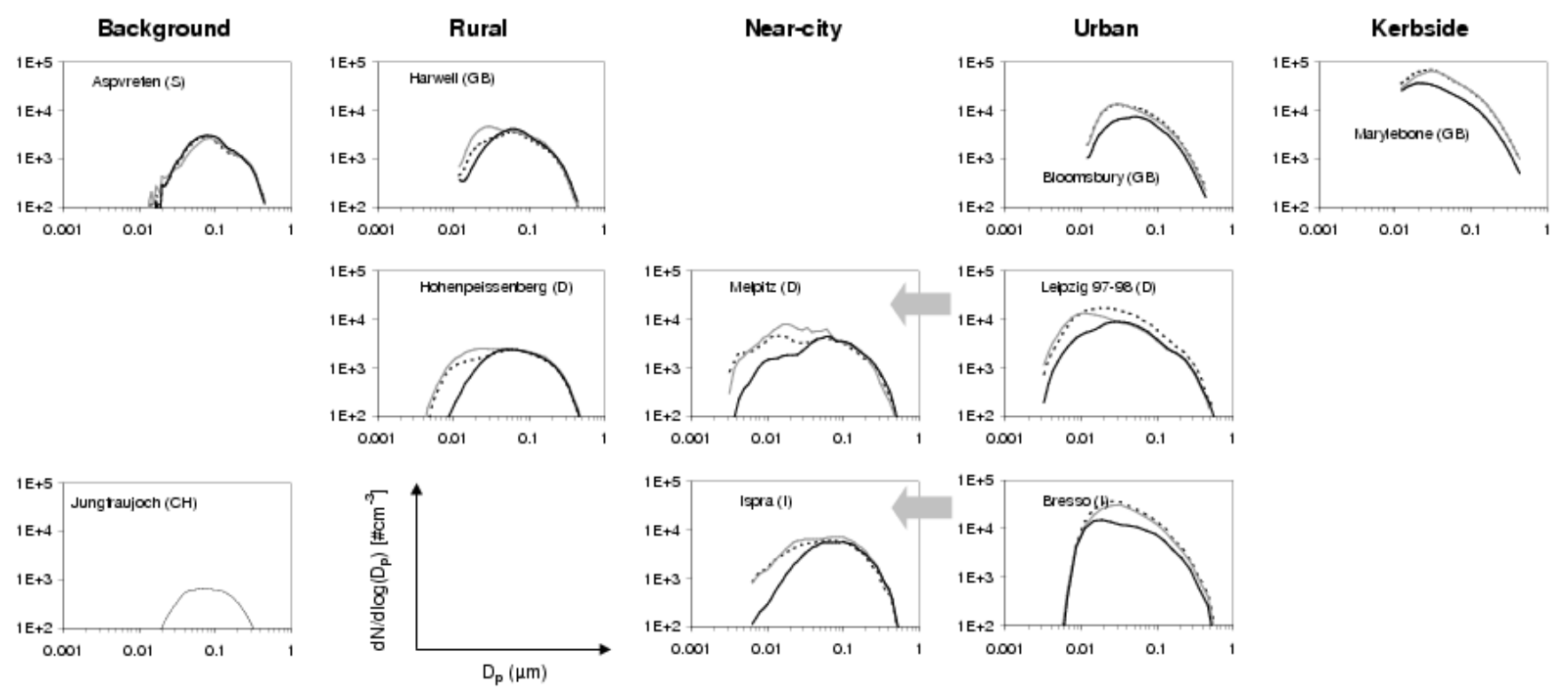

Fig. 6. Median particle number size distributions during summer, during morning hours (black dashed line), afternoon (grey full line) and night (black full line). From Van Dingenen et al. (2004).

Table 1. Table of $S\left(X_{i}\right)=\partial \ln N_{d} / \partial \ln X_{i}$ where $X_{i}$ is one of $N_{a}$, $r_{g}, \sigma_{g}, w, \varepsilon$. "Clean" indicates $N_{a}<1000 \mathrm{~cm}^{-3}$ and "Polluted" indicates $N_{a}>1000 \mathrm{~cm}^{-3}$. The ranges of $X_{i}$ are $w: 20 \mathrm{~cm} \mathrm{~s}^{-1}$ to $300 \mathrm{~cm} \mathrm{~s}^{-1}, N_{a}: 20 \mathrm{~cm}^{-3}$ to $3000 \mathrm{~cm}^{-3}, r_{g}: 0.03$ to $0.1 \mu m, \sigma: 1.3$ to $2.2, \varepsilon: 0.10$ to 1.00 .

\begin{tabular}{crrr}
\hline & All & Clean & Polluted \\
\hline$N_{a}$ & 0.88 & 0.92 & 0.73 \\
$r_{g}$ & 0.32 & 0.28 & 0.39 \\
$\sigma$ & -0.39 & -0.31 & -0.53 \\
$w$ & 0.29 & 0.18 & 0.47 \\
$\varepsilon$ & 0.11 & 0.09 & 0.13 \\
\hline
\end{tabular}

range of parameter space. Aerosol composition was represented in a simplified fashion by considering an ammonium sulphate and insoluble mix, and varying the mass fraction of ammonium sulphate, over the range 0.1 to 1.0 . The output was then used to examine the relative sensitivity of cloud drop size to the various input parameters using the model of Feingold (2003). Here we repeat this analysis for sensitivity of drop number concentration $N_{d}$. The sensitivities are defined as $S\left(X_{i}\right)=\partial \ln N d / \partial \ln X_{i}$ where $X_{i}$ is one of $N_{a}, r_{g}$, $\sigma_{g}, w$ or $\left.\varepsilon\right)$. In this form, values of $S\left(X_{i}\right)$ can be compared with one-another to assess their relative importance. Values of $S\left(X_{i}\right)$ for conditions similar to Feingold (2003) are given in Table 1.

Under clean conditions, arbitrarily defined as $N_{a}<1000 \mathrm{~cm}^{-3}, S\left(N_{a}\right)$ is close to its theoretical upper limit of 1 , indicating a high level of in-cloud activation. Sensitivity to $r_{g}$ and $\sigma_{g}$ under clean conditions is approxi- mately the same (although opposite in sign) whereas $S(w)$ is small. Under polluted conditions, the relative influence of $r_{g}, \sigma_{g}$ and $w$ on $N_{d}$ increases significantly while $S\left(N_{a}\right)$ decreases in importance. $S(\varepsilon)$ is relatively small compared to the other terms, although we caution that this term only reflects composition changes associated with the fraction of soluble material. The signs of $S(X i)$ are as expected; specific mention is made of $S\left(\sigma_{g}\right)$ which is negative because the tail of the distribution at large sizes results in activation of larger drops, and suppression of supersaturation which tends to suppress $N_{d}$. This combination of effects makes $S\left(\sigma_{g}\right)$ quite large, particularly under polluted conditions when the larger particles are abundant (e.g. O'Dowd et al., 1999, Sect. 3.1.2). Rissman et al. (2004) performed a more detailed analysis of the effect of various composition factors such as solubility and surface tension, as well as size distribution parameters. Their results were derived from analytical solutions, and presented in terms of a sensitivity relative to the sensitivity of drop number concentration to updraft velocity $\phi(\chi)=(\chi / w)\left(\partial N_{d} / \partial \chi\right) /\left(\partial N_{d} / \partial w\right)$, where $\chi$ is a composition factor such as organic mass fraction $\epsilon_{o}$. The authors concluded that when defined this way, sensitivity to composition factors $\phi(\chi)$ is highest for aerosol typical of marine condition, and increases with increasing updraught velocity. However, these are conditions under which supersaturation and activated fractions are high, and an increase in $w$ does not add many new drops $\left(\partial N_{d} / \partial w\right.$ is small). The appearance of $\partial N_{d} / \partial w$ in the denominator tends to increase $\phi(\chi)$. Thus at high $S$, even though $\phi(\chi)$ is large, composition effects may not be important in an absolute sense,. Because the individual sensitivities 
( $\partial N_{d} / \partial \chi$ and $\partial N_{d} / \partial w$ or their logarithmic equivalents) were not reported, it is difficult to compare their results to those of Feingold (2003) for overlapping parameter space. Both studies do however agree that $N_{d}$ is more sensitive to the size parameter $r_{g}$, and that $N_{d}$ is more sensitive to $r_{g}$ under polluted conditions.

The greater sensitivity of cloud droplet number to size compared to composition illustrates that the aerosol size must be captured as a primary pre-requisite. The sensitivity to the compositional complexities should only be investigated in the knowledge that the size and number information is likely to be equally important (or moreso). It should be noted that the treatment of composition does not address the sensitivity to composition changes with size and to varied composition at any one size; evidence for the prevalence of both being provided in the forthcoming sections. The sensitivity of activation and cloud droplet number to more detailed aspects of aerosol composition is discussed in Sect. 4.2.

\subsubsection{The composition of ambient aerosol}

Until recently, the vast majority of cloud modelling studies have conventionally assumed, implicitly or explicitly, that the soluble material in aerosol particles comprises inorganic components. The emerging complexity of ambient aerosol requires that this description is revisited.

It must be remembered that number of activated droplets is dependent on the number distribution of particles of a given type and not directly on the mass loading. Although the activation of an individual particle is dependent on its (soluble) mass, techniques which coarsely probe component distribution loadings by mass will not provide adequate insight to predict droplet number. Composition is likely to be important only in a limited size range: particles smaller than about $40 \mathrm{~nm}$ diameter are unlikely to activate into cloud droplets regardless of their composition and sufficiently large particles will almost always contain enough soluble material to activate. To predict droplet activation it is necessary to determine size-resolved composition in the 40 to $200 \mathrm{~nm}$ size range coupled to information about the mixing-state of the population. No single technique can currently provide all this information. This section reviews the available evidence for the composition of ambient particles from a range of studies and techniques. From combinations of these sources it should eventually be possible to adequately describe the aerosol composition distribution for purposes of CCN and possibly droplet number prediction.

Offline analyses of bulk particulate material collected by filter pack and impactor sampling have conventionally been used to determine aerosol mass composition. Applied analytical techniques can provide information ranging from detailed molecular speciation to aggregate lumped chemical functionality. These techniques have recently been supplemented by online instrumentation which may provide additional information to that available from offline techniques.
Some online instruments provide single particle composition information and hence information about which components co-exist in the same particles; their chemical mixing state. Others may provide component mass loadings with high size and time resolution. However, online techniques cannot currently provide as much detailed speciation information as may be available from offline techniques.

3.1.4.1 Offline bulk measurements showing the complexity of the atmospheric aerosol composition

This section presents such features of aerosol composition (organic and inorganic) which may be gained from offline analyses as relate to cloud activation, without attempting an exhaustive review. A summary of some available size segregated chemical compositions is also provided in a form suitable for cloud modelling purposes.

Despite the wide range of sampling and analytical techniques that have been employed, characterisation of aerosol chemical composition as a function of size is often still incomplete (Putaud et al., 2004). The chemical composition is diverse, complex and variable with location and condition. The particles comprise many inorganic and organic compounds ranging in solubility, density and, surface tension. Thus, comprehensive papers about the aspects of the chemical composition relevant to cloud formation are rare. In order to use Eq. (2), the chemical composition must be "translated" for cloud modelling purposes into concentration of molecules (organic and inorganic) dissolved in cloud droplets, total insoluble mass and, if present, the concentration of some "critical" species with limited solubility (or slightly soluble species). The degree of dissociation and $\mathrm{pH}$ may also be needed (Laaksonen et al., 1998; Lohmann et al., 2004); this is addressed further in Sect. 4. It should be noted that there are exceptions to these requirements when Eq. (1) is used, depending on how water activity is evaluated, see Sect. 4.4.

Soluble inorganic components are relatively well understood; the majority comprising a few inorganic salts (Heintzenberg, 1989), which are relatively wellcharacterised in terms of their hygroscopic properties (Clegg et al., 1998; Ansari and Pandis, 2000). The insoluble inorganic fraction can also be important (as in the case of dust aerosol from urban or natural sources) and many different component or element measurements are available. However, this information is difficult to directly interpret in terms of total insoluble mass.

Organic matter has been shown to represent an important fraction of the aerosol mass in different environments, and is routinely measured by means of thermal techniques (Liousse et al., 1996; Jacobson et al., 2000; Putaud et al., 2004). Organic carbon (OC) and Elemental carbon (EC) (or black carbon (BC)) are reported in terms of carbon mass and the transformation to aerosol mass is problematic without knowing the main chemical C structure (Turpin and Lim, 2001; Rus- 
sell, 2003). The assumption that $\mathrm{BC}$ belong to the insoluble fraction of the aerosol has been questioned by recent experiments showing that thermally refractory fractions of TC can be efficiently extracted with water (Yu et al., 2004; MayolBracero et al., 2002). Furthermore, OC/BC concentrations are strongly size dependent.

Experimental studies indicate that, in addition to the inorganic components, water-soluble organic compounds (WSOC) in atmospheric aerosol particles are also potentially important in clouds, and an understanding of organic partitioning in cloud droplets (whether dissolved or present as insoluble inclusions) is crucial to our understanding of their possible effects on cloud activation (see for example Facchini et al., 1999b; Jacobson et al., 2000; Kiss et al., 2001; Maria et al., 2003). WSOC, as opposed to inorganic aerosol components, comprise hundreds (or even thousands) of individual species (Saxena and Hildemann, 1996; Maria et al., 2004; Hamilton et al., 2004; Murphy, 2005; Kanakidou et al., 2005), each contributing a small fraction of the mass. Several studies of aerosol WSOC concentration and composition have been carried out (Zappoli et al., 1999; Facchini et al., 1999b; Kiss et al., 2001, 2002; Mayol-Bracero et al., 2002; Cavalli et al., 2004a,b; Putaud et al., 2004; Sullivan et al., 2004; Xiao and Liu, 2004). Molecular level identification and analysis is the traditional goal of aerosol organic analysis (for example IC: Falkovich et al., 2005; IEC-UV: Schkolnik et al., 2005; GC-MS: Graham et al., 2002; Pashynska et al., 2002; Carvalho et al., 2003; Ion et al., 2005), but such individual component approaches only account for a small fraction of the total aerosol and a long list of compounds present in very small concentration is usually provided. In addition to the analytical procedure, bulk sampling techniques which are frequently employed for such analyses are inappropriate for cloud activation purposes and size-segregated determination is necessary (Carvalho et al., 2003; Matta et al., 2003; Cavalli et al., 2004b; Putaud et al., 2004; Falkovich et al., 2005).

The representation of aerosol composition therefore presents a dilemma; it is evident that the aerosol WSOC cannot be correctly represented by molecules accounting for only a small fraction of the total carbon mass, but a representation of participating species is required for a fundamental prediction of cloud activation. Frequently, due to the its complexity, the WSOC chemical composition is reduced for modelling purposes to one or two "representative" species or surrogate molecules selected from the long list of compounds detected in the atmosphere. However, due to the complexity of WSOC and the wide range of physical properties relevant to activation, an arbitrary choice of representative compounds can fail in reproducing relevant physical and chemical properties. For the above reasons, a "realistic" representation of WSOC is necessary for cloud modelling purposes, but it is difficult to achieve through any individual analytical methodology or by choosing surrogate chemical compositions from a list of compounds detected in the aerosols.
Functional group analytical techniques provide an alternative approach to traditional individual compound speciation methods. These techniques analyse the different types of chemical structures (as for example total carboxylic groups, total carbonyls, etc.), but provide little or no information on the individual molecules (Decesari et al., 2000; Maria et al., 2002). Functional group methods have sometime been coupled to extraction-classification or separation techniques, providing a more comprehensive description of $\mathrm{OC}$ and being able to account for up to $90 \%$ of the WSOC mass (Decesari et al., 2001; Varga et al., 2001). In particular, in the functional group analysis approach proposed by Decesari et al. (2000), WSOC is separated into three main classes of compounds: neutral compounds (NC), mono/di-carboxylic acid (MDA) and polycarboxylic acids (PA). Quantitative measurements of WSOC by Total Organic Carbon (TOC) analyser and of proton concentration of the main functional groups contained in each of the three above mentioned classes by Proton Nuclear Magnetic Resonance (HNMR) can be used to formulate a set of a few "model" compounds representative of the whole WSOC (Fuzzi et al., 2001). A systematic technique for deriving model compounds for biomass burning aerosol collected in the Amazon has recently been submitted for publication (Decesari et al., 2006). Since the model compounds derived in this way reproduce quantitatively the average chemical structure of WSOC it can be argued that they may be used as bestguess surrogates in microphysical models involving biomass burning aerosol. Likewise, model mixtures of WSOC for many different types of aerosol in a range of locations are available or their definition is in progress:

. Urban aerosol, Bologna, Italy (Matta et al., 2003; Fuzzi et al., 2001),

. Dust aerosol, Monte Cimone, Italy (Putaud et al., 2004),

- Clean marine aerosol, Mace Head, Ireland (Cavalli et al., 2004b),

- Biomass burning aerosol, Rondonia, Brazil (Decesari et al., 2006),

- ACE Asia, Chinese outflow, Gosan, Jeju Island, Korea (Topping et al., 2004),

. Boreal forest aerosol, Hyytiälä, Finland (Cavalli et al., 2004a; Decesari et al., 2006).

In summary, only a few studies have provided information concerning both inorganic and organic aerosol chemical composition which can be directly used by cloud models. These papers provide a comprehensive description of the chemical composition of different aerosol types as a function of size (Chan et al., 1999; Zappoli et al., 1999; Pakkanen et al., 2001; Putaud et al., 2000; Temesi et al., 2001; Maria et al., 2003; Sellegri et al., 2003; Cabada et al., 2004; Chio et al., 2004; Sardar et al., 2005). 
Section 6.1 presents results from such analyses as described above from a variety of locations worldwide.

In addition to the functional groupings identified above, there are a number of classes of nitrogen-containing compounds which are relatively abundant in airborne particulates (refs). Initially thought primary in origin, and hence generally concentrated in the coarse mass modes, proteins, peptides, amino acids and related amino-compounds are reasonably documented in terms of their mass loadings (Saxena and Hildemann, 1996; Miguel et al., 1999; Zhang and Anastasio, 2001, 2003; Zhang et al., 2002b; Mace et al., 2003a,b,c; Kuznetsova et al., 2005; Matsumoto and Uematsu, 2005; Poeschl, 2005). More recent postulations suggest that secondary processes may contribute to their transformation (Franze et al., 2005). Studies of these compounds in particulate matter, hydrometeors, and precipitation have reported high concentrations, indicating that they account for a major mass fraction of water-soluble organic carbon and may be present in significant numbers of fine particles. These compounds are known to act as surfactants and are thereby likely to influence the interaction of atmospheric aerosol particles with water vapor through the surface tension term in Eq. (1) or through other surface effects (see Sect. 4.1.4). The roles of these compounds are discussed in Sect. 4.1.9.

\subsubsection{Online techniques}

Whilst useful detailed chemical composition information may be obtained by bulk sampling and offline analysis, there are notable drawbacks to the approach. Coe and Allan (2005) have reviewed a range of online mass spectroscopic methods which have been developed over the last decade. These fall into two principal types. The first are laser-based systems that ablate single particles and obtain a mass spectral fingerprint of a single particle. These instruments, reviewed in detail by Noble and Prather (2000), offer a method of probing the mixing state of aerosol particle but at present cannot deliver quantitative assessments of the mass of individual components. The second type uses thermal volatilisation to vaporise the particles followed by electron bombardment to ionise the neutral gas. This latter method, to-date most widely employed in the Aerodyne Aerosol Mass Spectrometer (AMS), was first described by Jayne et al. (2000). It is able to provide mass loadings of non refractory components in the submicron range and deliver mass size distributions of key species type, Jimenez et al. (2003) first demonstrated the methodology for mass quantification and Allan et al. (2003b) described the errors. A principal constraint of this approach has been the limited availability of single particle (and hence mixing state) information.
3.1.4.2.1 Online measurement techniques providing ensemble mass composition distributions

A large number of field experiments have now been carried out with the Aerodyne AMS in a wide variety of different sampling environments. In urban environments across North America and Europe (Allan et al., 2003a; Drewnick et al., 2004; Boudries et al., 2004; Alfarra et al., 2004; Zhang et al., 2005b), the sub $100 \mathrm{~nm}$ diameter particles are dominated by organic material which has been correlated with combustion sources (e.g. Allan et al., 2003b). Even in locations with large sulphate sources and significant nucleation of sulphuric acid, the organic fraction of the sub $100 \mathrm{~nm}$ particles approaches 90\% (Zhang et al., 2005b). Where photochemistry is efficient, inorganic species are also observed at these smaller sizes suggesting condensation is occurring onto these freshly produced particles (e.g. Zhang et al., 2004; Alfarra et al., 2004), increasing their hygroscopicity. The mass spectral fingerprint of these aerosols demonstrates that they are principally hydrocarbon-like in nature and, so far as AMS fragmentation is concerned, can be represented by lubricating oil and fresh diesel exhaust (Canagaratna et al., 2004). The high organic content at sizes around the droplet activation threshold suggests that the effect of organics on warm cloud activation may be much larger than their contribution to the overall mass budget of sub-micron particles. For example, with reference to all panels other than (f) and (h) in Fig. 7, the organic to inorganic ratio below $200 \mathrm{~nm}$ dry diameter is clearly higher than the average ratio across the submicron distribution. It may be expected that any effect that organic components have on activation properties is exacerbated here. Since particles greater than $200 \mathrm{~nm}$ are likely to activate at any reasonable updraught velocity, droplet number is most likely to be influenced by composition effects for the very fine particles below $200 \mathrm{~nm}$ in diameter. However, even in urban environments the largest fraction of the non refractory particle mass arises in the accumulation mode and is composed of organic and sulphate aerosol which from their modal and temporal behaviour can be considered to be largely internally mixed (Alfarra et al., 2004). This inference about the mixing state may be important for the activation of accumulation mode particles.

Further from the source region the sub $100 \mathrm{~nm}$ becomes less important and the aerosol population in the regional background in many mid latitude continental regions becomes dominated by the accumulation mode composed of organic and sulphate components, which show a widely varying ratio of between around 0.2 and 0.8 depending on source. Where sulphate is buffered by adequate ammonium, or sulphur sources are few, ammonium nitrate is observed in the same mode (Alfarra et al., 2004; Allan et al., 2003a); this is more common in the western parts of North America and in Western Europe. Measurements using the AMS in the background atmosphere have so far been limited to the Northern Hemisphere mid-latitudes between $30^{\circ}$ and $60^{\circ} \mathrm{N}$ but at all 


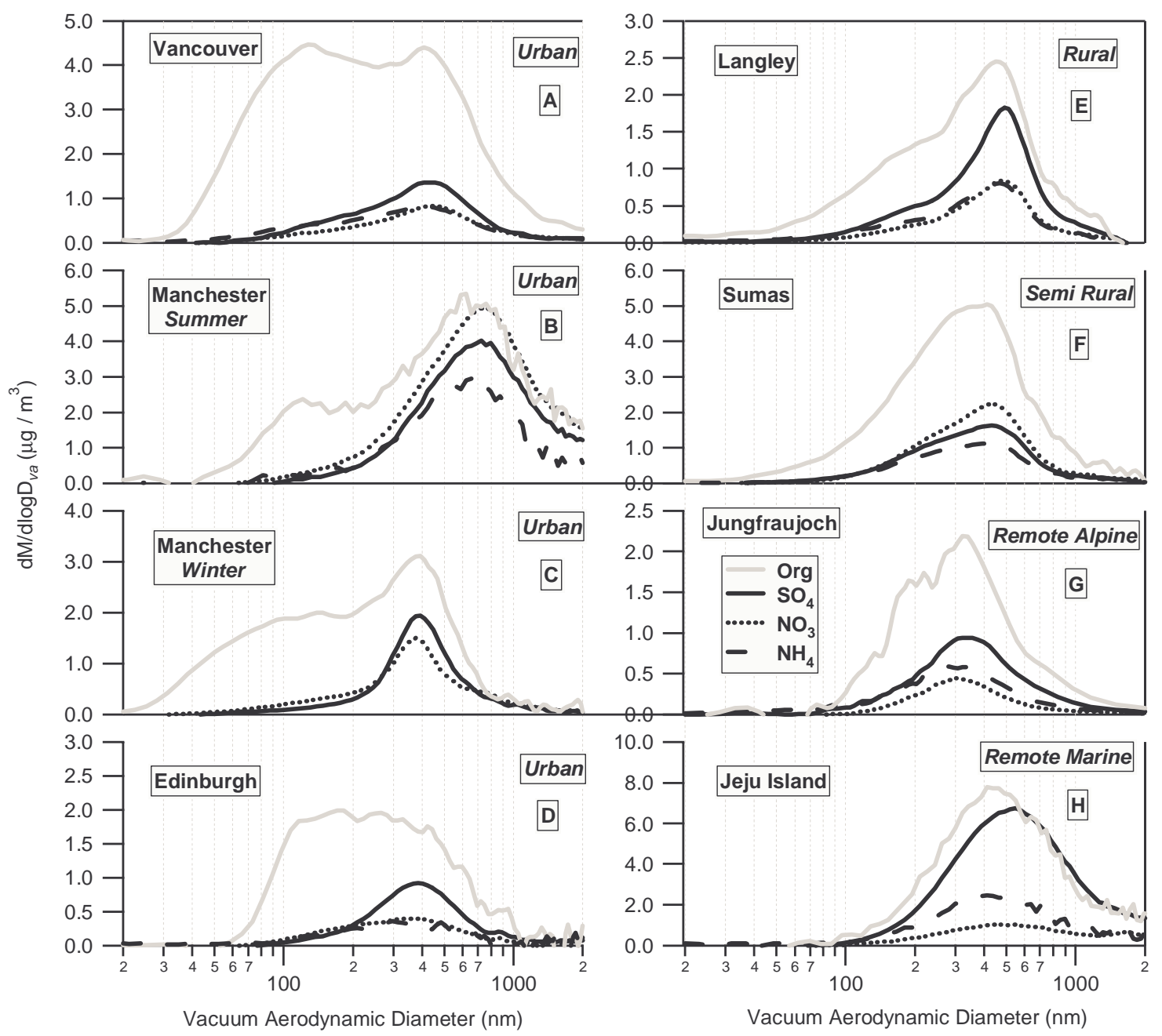

Fig. 7. AMS component mass distributions in a range of locations (after McFiggans et al., 2005). The primary difference between the distributions measured close to strong sources (left panels) and more remote distributions (right panels) is the presence in the polluted cases of a significant mass loading in an externally-mixed mode dominated by organic compounds around the threshold dry size for cloud activation. This mode corresponds to the less hygroscopic mode observed in urban and polluted continental air (see Sect. 3.2.1, Table 3) and dominates when translated into number space.

locations a single mass mode centred at between 350 and $400 \mathrm{~nm}$ diameter and comprising organic and acidified sulphate aerosol has been observed (Allan et al., 2004 (northern California); Topping et al., 2004 (Jeju Is., Korea); Schneider et al., 2004 (Crete)); Rupakheti et al., 2005 (rural Ontario)). The Allan et al. (2004) and Topping et al. (2004) studies show that even in marine environments the organic-acid sulphate aerosol usually dominates the mass loading of the accumulation mode below $500 \mathrm{~nm}$ but at larger sizes sea spray derived aerosol is increasingly important and dominates in the supermicron size range, results from a mountain station in the Swiss Alps (see Sect. 6.3) have shown that whilst nitrate and organic material dominates particulate driven up to the site by orographic winds, acidified sulphate aerosol dominates in the lower free troposphere, though organic mate- rial is also present. Bahreini et al. (2003) measured elevated acidic sulphate concentrations along with a comparable loading of highly oxygenated organic components in multiply stratified layers in the lower free troposphere during ACEASIA using an airborne AMS. Figure 7 shows the average size distributed mass loading of the key non refractory components of submicron aerosol measured by AMS at a range of sites from urban sources to the remote continental background.

The mass spectral fingerprints of the organic fraction of the background continental accumulation mode aerosol are very similar from site to site. Zhang et al. (2005a) have developed a two component approach to interpretation of the AMS mass spectra that, for localities in regions of fresh or aged pollution sources appears to account for over $90 \%$ of 
the variance observed. For northern mid-latitudes, where most of these analyses have been conducted, the characteristic component mass distributions in more aged airmasses show that the accumulation mode organics are largely present in almost identical modal sizes as inorganic components - i.e. that they have some degree of internal mixing. It is precisely these airmasses which show the mass fragmentation patterns with a characteristically high degree of oxygenation. This implies that, when present, the oxygenated organics may be approximated as internally mixed with the accumulation mode inorganics. In contrast, urban-influenced air, exhibiting mass distributions with an essentially externally-mixed high Aitken mode organic contribution are those airmasses with a more hydrocarbon-like fragmentation pattern. The mass of such organics may probably be apportioned by number to the Aitken mode distribution: HTDMA studies (see Sect. 3.2.1) confirm that this is frequently the case in urbaninfluenced air with the observation of a separate externallymixed less hygroscopic or even hydrophobic mode. Unfortunately, this representation of the aerosol distribution around the threshold dry size for droplet activation is highly complex and at any size where the Aitken and accumulation modes overlap, one is likely to find an external mixture of hydrocarbon like, organic dominated, low hygroscopicity particles and oxygenated organic compounds largely co-existing in the same particles as the inorganic components. The number ratio of these particle populations will change with size as the local Aitken and background accumulation mode contributions vary. An AMS, fitted with a Time of Flight Mass Spectrometer, has been shown to be able to provide quantitative measurement of single particle chemical composition as a function of size for the non-refractory component of the particles with mass loading in agreement with a quadrupole version of the same instrument (Drewnick et al., 2005). This and other similar advancements may enhance our future capability to interpret aerosol-cloud interactions.

3.1.4.2.2 Online measurement techniques providing single particle information

Online single-particle techniques may exhibit many of the same intrinsic advantages as those described above. In addition they can provide single particle mixing-state information at diameters close to the activation threshold dry radius, single particle cluster ensemble information and, using upstream separation, single particle information on droplet residuals and interstitial aerosol to derive number scavenging ratios by composition. However, the single particle techniques are not as quantitative in describing bulk composition as the AMS results described above.

On-line single particle measurements have been made of tropospheric particles larger than about $0.15 \mu \mathrm{m}$ diameter: a little larger than the sizes where particle composition is most important for activation (see Sect. 3.1.1. Except in a few source regions, most tropospheric particles contained at least some of both organics and inorganic materials. For example, in Atlanta 70 to $99 \%$ of the measured particles contained organics and 90 to $98 \%$ contained sulphate (Lee et al., 2002). Even in a very clean marine boundary layer the majority of the particles contained both sea salt and organics (Middlebrook et al., 1998). Indeed, it is extremely difficult to prepare pure sulphuric acid particles in the laboratory without organic contamination (Middlebrook et al., 1997). Conversely, the widespread oxidation of $\mathrm{SO}_{2}$ to $\mathrm{H}_{2} \mathrm{SO}_{4}$ (by $\mathrm{OH}$ in the sunlit atmosphere) followed by condensation onto particles ensures that any particle that has spent much time in the atmosphere has acquired at least a small percentage of sulphate.

Figure 8 shows that a large fraction of particles throughout the troposphere contain both sulphate and organics. This does not imply that the particles in a given volume of air are all uniformly mixed, just that most contain measurable amounts of both organics and ionic species. The similarity of the organic peaks from one mass spectrum to the next suggests that most particles contain a mixture of organics as well. Entropy also provides a thermodynamic incentive for at least some of the thousands of organic compounds to mix together in single particles. The single particle data are quite consistent with mountaintop tandem mobility analyser data. Nessler et al. (2003) reported "Most of the time, the station was located in the free troposphere, and the shape of the HTDMA spectra was primarily characterized by a narrow monomodal size distribution (only $7 \%$ of the particles experienced a smaller hygroscopic growth than the particles in the dominant mode). .... Exceptions from this behaviour were limited to short time periods when the station was influenced by local pollution due to construction work or dust plumes from North Africa (Saharan dust)."

These measurements have definite implications for warm cloud activation. If almost all particles contain some ionic species then completely hydrophobic particles will be rare except in special regions. This is borne out by tandem differential mobility studies (see Sect. 3.2.1). Even relatively small amounts of ionic material can allow the activation of organic particles (Ervans et al., 2004; Lohmann et al., 2004), see Sect. 3.2.1, though the abundance and nature of organics will affect both equilibrium water content and droplet growth kinetics (Sect. 4.1).

\subsubsection{Use of combinations of techniques}

It is evident that each of the techniques described above may provide distinct useful contributions to our understanding of the effect of aerosol composition on droplet activation and that considerable advantage may be derived from simultaneous interpretation of data from combinations of offline and online composition analyses. Several field studies have deployed impactor and AMS measurements of composition in a variety of locations. Topping et al. (2004) demonstrated that the AMS organic mass was in agreement with both the 


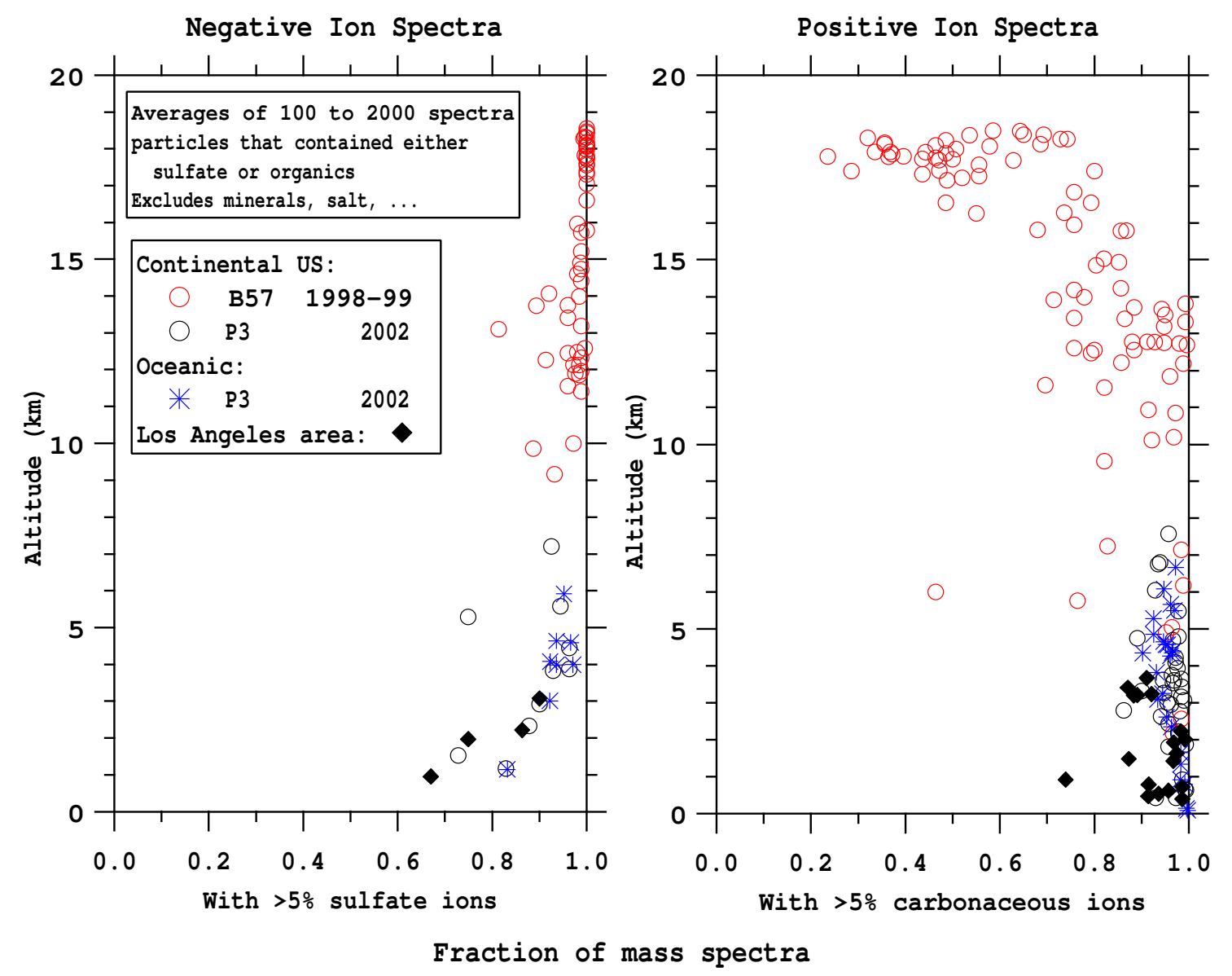

Fig. 8. The fraction of single particle mass spectra containing signals from sulfate and organic compounds. Most of the measured particles were between 0.2 and $0.9 \mu \mathrm{m}$ diameter. The stratosphere is the only place where most of the particles give no organic signal. Many of the particles below $3 \mathrm{~km}$ with no sulfate signal were nitrate particles, so they also were organic-inorganic mixtures. Negative ion spectra are very sensitive to sulfate and positive ion spectra are sensitive to organics, so particles with as little as $1 \%$ sulfates or organics may contribute $5 \%$ of the ions.

impactor-derived submicron water soluble organic carbon and total organic carbon, within experimental uncertainties in the clean and continentally-influenced marine boundary layer. However, given the methodology for extracting the water soluble component is relatively vigorous, it may not be that the water extracted organic carbon is in water solution in the ambient aerosol but may be concentrated at the surface or be partially immiscible. These properties, whilst crucial for cloud activation are poorly understood at present (see section on theoretical treatment of limited solubility, Sect. 4.1.3). McFiggans et al. (2005) likewise demonstrated that AMSand impactor-derived inorganic and organic aerosol loadings were of comparable magnitude in the polluted continental boundary layer. The authors combined the techniques using mobility and aerodynamic sizing instrumentation to improve size and time resolution of the impactor-derived detailed organic functionality analyses. This was combined with HTDMA analyses (see Sect. 3.2.1) to attempt to reconcile the composition and sub-saturated water content (see Sect. 5.1) using a model incorporating the primitive form of the Köhler Eq. (1) (see Sect. 4.4).

\subsection{Measurements of derived properties of atmospheric aerosol particles and populations}

Derived properties directly relevant to cloud activation may be separated into those which are intrinsic properties of the constituent particles of a population, depending on their fundamental properties such as composition and size and those which are composite distribution properties. We shall concentrate on two derived properties; i) hygroscopicity, an example of the former and ii) the ability to act as a CCN, an example of a distribution-dependent property when provided as a CCN activity spectrum. Though the composition mixingstate is not strictly a derived property, when probed in terms of hygroscopicity it may provide useful information on the effect of the aerosol distribution on cloud activation and is briefly considered here. 
Table 2. Hygroscopic growth factors of various "pure" substances.

\begin{tabular}{|c|c|c|c|}
\hline \multicolumn{4}{|c|}{ GF at $90 \% R H$} \\
\hline Substance & $80 \mathrm{~nm}$ & Flat surface & References \\
\hline$\left(\mathrm{NH}_{4}\right)_{2} \mathrm{SO}_{4}$ & 1.68 & 1.74 & Topping et al. (2004) \\
\hline $\mathrm{NH}_{4} \mathrm{HSO}_{4}$ & $1.72^{1}$ & $1.77^{1}$ & \\
\hline $\mathrm{H}_{2} \mathrm{SO}_{4}$ & $1.66^{1}$ & $1.70^{1}$ & \\
\hline $\mathrm{NH}_{4} \mathrm{NO}_{3}$ & 1.77 & 1.85 & \\
\hline $\mathrm{NaCl}$ & 2.34 & 2.41 & \\
\hline fresh mineral dust & $<1.05$ & - & Vlasenko et al. (2005) \\
\hline $\begin{array}{l}\text { fresh diesel engine exhaust } \\
\text { (soot) }\end{array}$ & $<1.05$ & - & Weingartner et al. (1997) \\
\hline $\begin{array}{l}\text { fresh petrol engine exhaust } \\
\text { dominated by volatile } \\
\text { organic compounds }\end{array}$ & $<1.05$ & - & Weingartner et al. (1995) \\
\hline biomass burner exhaust & up to $1.65^{2}$ & - & Pagels et al. (2003) \\
\hline $\begin{array}{l}\text { secondary organic aerosol } \\
\text { (reaction chamber experiments) }\end{array}$ & $\begin{array}{c}1.07-1.14 \\
(\text { at } 85 \% R H)\end{array}$ & - & $\begin{array}{r}\text { Saathoff et al. (2003) } \\
\text { Virkkula et al. (1999) } \\
\text { Baltensperger et al. (2005) } \\
\text { Cocker et al. (2001) }\end{array}$ \\
\hline humic-like substances & $1.08-1.20$ & - & $\begin{array}{r}\text { Chan and Chan (2003) } \\
\text { Gysel et al. (2004) } \\
\text { Brooks et al. (2004) }\end{array}$ \\
\hline organic acids & $1.00-1.70^{3}$ & - & $\begin{array}{l}\text { Wise et al. (2003) and } \\
\text { references therein }\end{array}$ \\
\hline
\end{tabular}

${ }^{1}$ Divided by $G F_{D}=1.22$ for $\mathrm{H}_{2} \mathrm{SO}_{4}$ and 1.03 for $\mathrm{NH}_{4} \mathrm{HSO}_{4}$. at $\mathrm{RH}=10 \%$.

2 Exhaust from large (1 to $6 \mathrm{MW}$ ) high efficiency grate burners leave particles composed of the inorganic residue only. Particles from open or smouldering flames with significant amounts of organic and elemental carbon are expected to have smaller $G F_{D}$ values.

${ }^{3} G F_{D}$ similar to inorganic salts are reported for multifunctional organic acids and organic salts, whereas monoacids are generally not or only slightly hygroscopic.

\subsubsection{Hygroscopicity}

The ratio of the diameter of a particle at a known elevated relative humidity $(R H)$ to that when nominally dry is the hygroscopic diameter growth factor $\left(G F_{D}\right)$. Particle $G F_{D}$ values at $R H$ below saturation are related to their $\mathrm{CCN}$ activation behaviour (Sect. 3.2.2) through Köhler theory (Sect. 2) and hence are important in understanding the cloud activation of aerosol particles. Furthermore, $G F_{D}$ measurements also provide insight into the aerosol chemical mixing state.

Ambient $G F_{D}$ measurements are commonly determined using a Hygroscopicity Tandem Differential Mobility Analyser (HTDMA, Liu et al., 1978; Rader and McMurry, 1986). A size fraction, selected from the dried ambient aerosol with a Differential Mobility Analyser (DMA, Liu and Pui, 1974) is exposed to well-defined elevated $R H$ in a humidifier and the grown equilibrium size distribution is determined by scanning a second humidified DMA connected to a particle counter across an appropriate size range. Inversion algorithms must be applied to the measured distributions to obtain the effective growth distribution of the aerosol owing to the finite width of the DMA transfer functions during dry particle selection and grown particle detection. Most widespread are the TDMAFIT algorithm (Liu et al., 1978; Rader and McMurry, 1986) (Stolzenburg and McMurry, 1988), accounting for the transfer function width of both DMAs and algorithms, where only the second DMA (and particle counter response) is inverted (Stratmann et al., 1997; Voutilainen et al., 2000; Collins et al., 2002). Recently, Cubison et al. (2005) have introduced an alternative approach, where the TDMA measurement is inverted into contributions from fixed classes of narrow growth factor ranges. The resolution of inverted growth distributions is limited by measurement uncertainties and knowledge of the exact TDMA transfer function. 
Table 3. Hygroscopic behaviour of Aitken mode particles in various environments.

\begin{tabular}{|c|c|c|c|c|c|c|c|}
\hline \multicolumn{8}{|c|}{ upper Aitken mode size range $(\sim 50-80 \mathrm{~nm})$} \\
\hline & & dominant mode & & & second mode & & \\
\hline & $G F_{D}(90 \%)$ & $\begin{array}{l}\text { frequency } \\
\text { of } \\
\text { occurrence }\end{array}$ & fraction & $G F_{D}(90 \%)$ & $\begin{array}{l}\text { frequency } \\
\text { of } \\
\text { occurrence }\end{array}$ & fraction & References \\
\hline Urban & $1.15-1.43$ & $\begin{array}{l}\text { up to } \\
100 \%\end{array}$ & $11-90 \%$ & $1.00-1.12$ & $\begin{array}{l}\text { up to } \\
100 \%\end{array}$ & $10-89 \%$ & $\begin{array}{l}\text { McMurry and Stolzenburg (1989) } \\
\text { Cocker et al. (2001) } \\
\text { Zhang et al. (1993) } \\
\text { Baltensperger et al. (2002) } \\
\text { Chen et al. (2003) } \\
\text { Ferron et al. (2005) }\end{array}$ \\
\hline $\begin{array}{l}\text { Continental } \\
\text { Polluted }\end{array}$ & $1.32-1.53$ & $90-100 \%$ & $28-97 \%$ & $1.05-1.15$ & $10-90 \%$ & $3-72 \%$ & $\begin{array}{l}\text { Svenningsson et al. (1992) } \\
\text { Zhang et al. (1993) } \\
\text { Pitchford and McMurry (1994) } \\
\text { Svenningsson et al. (1994) } \\
\text { Svenningsson et al. (1997) } \\
\text { Swietlicki et al. (1999, 2000) } \\
\text { Hämeri et al. (2001) } \\
\text { Busch et al. (2002) } \\
\text { Boy et al. (2003) } \\
\text { Ferron et al. (2005) }\end{array}$ \\
\hline $\begin{array}{l}\text { Free } \\
\text { Troposphere }\end{array}$ & $1.40-1.55$ & $100 \%$ & $75-90 \%$ & $\begin{array}{r}\text { bimodal g } \\
\text { observed at } \\
\text { a.s.l. in }\end{array}$ & $\begin{array}{l}\text { Wth }\left(\mathrm{GF}_{D} \sim\right. \\
\text { zaña, Teneri } \\
7 \% \text { of clean }\end{array}$ & $\begin{array}{l}-1.6) \\
2357 \mathrm{~m} \\
\text { ses }\end{array}$ & $\begin{array}{l}\text { Swietlicki et al. (2000) } \\
\text { Weingartner et al. (2002) }\end{array}$ \\
\hline $\begin{array}{l}\text { Biogenically } \\
\text { dominated }\end{array}$ & $1.15-1.20$ & $>97 \%$ & $87-95 \%$ & $\sim 1.07$ & $15-35 \%$ & $<12 \%$ & $\begin{array}{l}\text { Zhou et al. (2002) } \\
\text { Rissler et al. (2004) }\end{array}$ \\
\hline $\begin{array}{l}\text { Remote } \\
\text { Marine }\end{array}$ & $1.42-1.75$ & $100 \%$ & $>75 \%$ & $2.05-2.13$ & $3-7 \%$ & $<7 \%$ & $\begin{array}{l}\text { Berg et al. (1998b) } \\
\text { Swietlicki et al. (2000) } \\
\text { Zhou et al. (2001) }\end{array}$ \\
\hline
\end{tabular}

$G F_{D}$ values of individual aerosol compounds are summarised in Table 2. Inorganic salts are highly hygroscopic with $\mathrm{NaCl}$ at the upper end of the growth scale. Nonhygroscopic particles include fresh mineral dust, fresh diesel engine exhaust (soot - mainly elemental carbon and waterinsoluble organic species, "WINSOL") and fresh petrol engine exhaust (WINSOL-dominated). (Note, that Petzold et al. (2005) recently reported the strong dependence of $G F_{D}$ and $\mathrm{CCN}$ activation of gas turbine combustor particles on combustion conditions and resultant content of volatile and involatile organic compounds and sulphuric acid). Characterisation of the water-soluble organic aerosol fraction is more complicated. Pure organic acids range from non-hygroscopic to $G F_{D}$ values similar to inorganic salts. However, $G F_{D}$ of $\sim 1.15$ at $90 \% R H$, as observed for secondary organic aerosols formed in reaction chamber experiments and for humic-like substances, are probably more representative of organic mixtures in aged atmospheric particles, except for urban aerosols, where WINSOL are likely to be dominant. $G F_{D}$ values of biomass burning particles may be as high as 1.65 at $90 \% R H$ for highly efficient grate burners leaving only inorganic residue. However, particles from open or smouldering flames with significant amounts of organic and elemental carbon are expected to have a smaller $G F_{D}$.

An overview of growth modes of atmospheric aerosols observed in various environments is given in Table 3 for the upper Aitken mode size range $(\sim 50-80 \mathrm{~nm})$ and Table 4 for the lower accumulation mode size range $(\sim 100-150 \mathrm{~nm})$. These size ranges were chosen to cover the critical size range for $\mathrm{CCN}$ activation in clouds. The trend is for $50 \mathrm{~nm}$ particles to be less hygroscopic than particles at 150 or $165 \mathrm{~nm}$. For the studies listed in the table the growth factor at about $150 \mathrm{~nm}$ averages about 0.1 larger than at $50 \mathrm{~nm}$. This may be because of greater organic content near $50 \mathrm{~nm}$ (see section on bulk composition). Whatever the reason, less water uptake at smaller sizes implies slightly less sensitivity of activation to updraught velocity, since a faster updraught that activates more particles will encounter particles that are less hygroscopic. For example, if an air parcel that would activate $120 \mathrm{~nm}$ particles in a given updraught instead encounters an 
Table 4. Hygroscopic behaviour of accumulation mode particles in various environments.

\begin{tabular}{|c|c|c|c|c|c|c|c|}
\hline \multicolumn{8}{|c|}{ lower accumulation mode size range $(\sim 100-150 \mathrm{~nm})$} \\
\hline & & dominant mode & & & second mode & & \\
\hline & $G F_{D}(90 \%)$ & $\begin{array}{l}\text { frequency } \\
\text { of } \\
\text { occurrence }\end{array}$ & fraction & $G F_{D}(90 \%)$ & $\begin{array}{l}\text { frequency } \\
\text { of } \\
\text { occurrence }\end{array}$ & fraction & References \\
\hline Urban & $1.23-1.50$ & $100 \%$ & $16-90 \%$ & $1.00-1.14$ & $\begin{array}{l}\text { up to } \\
100 \%\end{array}$ & $10-84 \%$ & $\begin{array}{l}\text { McMurry and Stolzenburg (1989) } \\
\text { Cocker et al. (2001) } \\
\text { Zhang et al. (1993) } \\
\text { Baltensperger et al. (2002) } \\
\text { Chen et al. (2003) } \\
\text { Santarpia et al. (2004) } \\
\text { Ferron et al. (2005) }\end{array}$ \\
\hline $\begin{array}{l}\text { Continental } \\
\text { Polluted }\end{array}$ & $1.41-1.64$ & $100 \%$ & $37-100 \%$ & $1.03-1.18$ & $10-90 \%$ & $2-63 \%$ & $\begin{array}{l}\text { Svenningsson et al. (1992) } \\
\text { Covert and Heintzenberg (1993) } \\
\text { Zhang et al. (1993) } \\
\text { Pitchford and McMurry (1994) } \\
\text { Svenningsson et al. (1994) } \\
\text { Svenningsson et al. (1997) } \\
\text { Swietlicki et al. (1999, 2000) } \\
\text { Hämeri et al. (2001) } \\
\text { Busch et al. (2002) } \\
\text { Boy et al. (2003) } \\
\text { Ferron et al. (2005) }\end{array}$ \\
\hline $\begin{array}{l}\text { Free } \\
\text { Troposphere }\end{array}$ & 1.62 & $100 \%$ & $\sim 85 \%$ & $<1.3$ & - & $\sim 15 \%$ & Weingartner (2002) \\
\hline $\begin{array}{l}\text { Biogenically } \\
\text { dominated }\end{array}$ & $1.20-1.25$ & $>93 \%$ & $90-96 \%$ & $\sim 1.08$ & $\sim 25 \%$ & $<7 \%$ & $\begin{array}{l}\text { Zhou et al. (2002) } \\
\text { Rissler et al. (2004) }\end{array}$ \\
\hline $\begin{array}{l}\text { Remote } \\
\text { Marine }\end{array}$ & $1.47-1.78$ & $100 \%$ & $>80 \%$ & $2.06-2.14$ & $13-40 \%$ & $<15 \%$ & $\begin{array}{l}\text { Berg et al. (1998b) } \\
\text { Swietlicki et al. (2000) } \\
\text { Zhou et al. (2001) }\end{array}$ \\
\hline
\end{tabular}

updraught which would activate particles of $75 \mathrm{~nm}$ of the same composition, the $75 \mathrm{~nm}$ particles would not activate since they are not of the same composition but are less hygroscopic. Hence an even greater updraught would be required to activate the $75 \mathrm{~nm}$ particles. The compilations in Tables 3 and 4 are rather a reflection of ranges of observed values than a full climatology. The tables contain the mean growth factor, the frequency of occurrence and the fraction of total particles observed for the dominant and the second most important growth mode. Reduction of ambient growth distributions to two growth modes may be an oversimplification in some cases, but many studies report only two modes, and the actual growth distribution may often be reasonably well represented by two growth modes in conjunction with corresponding growth spread factors.

Urban aerosols are often characterised by an external mixture (see Sect. 3.2.3) of distinct modes of intermediately hygroscopic background particles and marginally hygroscopic particles from local emissions. The abundance of both modes is often similar but varies strongly from location to location and as a function of time. $G F_{D}$ of the intermediately hygroscopic background particles tend to be somewhat smaller than the dominant mode in continental polluted environments, indicating the hygroscopicity of the background particles in urban areas is reduced by the presence of less hygroscopic compounds.

Continental polluted aerosols have been investigated in many locations ranging from near-urban to remote and hence the corresponding growth characteristics provided in Tables 3 and 4 cover a wide range of air masses. The readily hygroscopic background mode generally dominates continental polluted aerosols, though considerable fractions of marginally hygroscopic particles may be found in proximity to urban areas. The background mode is always present, whereas marginally hygroscopic particles are not always found. There is some trend of decreasing fraction of marginally hygroscopic particles with increasing distance from major anthropogenic sources, but they may still be 
found after $5000 \mathrm{~km}$ or 5 days of transport (Heintzenberg and Covert, 1987). The conditions and processes favouring the removal or transformation of marginally hygroscopic particles are still to be elucidated.

Only a few of the HTDMA measurements of free tropospheric aerosols unaffected by recent anthropogenic influence taken as part of the Global Atmosphere Watch (GAW) programme at the Izaña (Tenerife, $2367 \mathrm{~m}$ a.s.l.) and Jungfraujoch (Switzerland, $3580 \mathrm{~m}$ a.s.l.) stations have been published. Values shown in Tables 3 and 4 include only periods where the stations were unaffected by the mixed planetary boundary layer, and they exclude Sahara dust events connected with externally mixed mineral dust particles as occasionally observed at the Jungfraujoch station (Weingartner et al., 2001). The free tropospheric aerosol is dominated by readily hygroscopic particles. Bimodal growth distributions with $G F_{D} \sim 1.4$ and $\sim 1.6$ were frequently observed for Aitken mode particles at the Izaña station, whereas narrow growth distributions were characteristic for the Jungfraujoch station. The trend of increasing growth factors from urban through continental polluted to free tropospheric air masses indicates that, with the progression of atmospheric ageing processes, readily hygroscopic inorganic salts dominate moderately hygroscopic organic compounds. This is consistent with the observation that accumulation mode particles are generally more hygroscopic than Aitken mode particles, indicating that the increased hygroscopicity of accumulation mode particles is mainly due to their increased fraction of inorganic salts. Such dominance of inorganic compounds and low contribution of even highly oxygenated organic components to equilibrium water content (and hence $\left.G F_{D}\right)$ in aged aerosol was also reported by McFiggans et al. (2005).

Biogenically dominated aerosols were investigated in the pristine Amazonian rain forest. Corresponding values reported in Tables 3 and 4 exclude episodes of clear influence from biomass burning. These aerosols are dominated by organic compounds and hence the growth factors are moderate. A minor fraction of marginally hygroscopic particles is occasionally present. Air masses influenced by recent or aged biomass burning showed increased hygroscopic growth factors of the dominant mode or presence of externally mixed particles with larger growth factors, but a larger fraction of marginally hygroscopic particles was also observed. This indicates that efficient biomass burning releases readily hygroscopic inorganic residue, but that also marginally hygroscopic particles are produced during the smouldering phase. Impacts of biomass burning have been reported elsewhere showing both trends of increasing (Cocker et al., 2001) and decreasing hygroscopicity (Carrico et al., 2005). Measurements in other locations with significant biogenic emission also show moderate hygroscopic growth factors associated with large organic aerosol fractions (Hämeri et al., 2001; Aklilu and Mozurkewich, 2004; Carrico et al., 2005).
Hygroscopic properties of remote marine aerosols have been investigated in the Pacific, Southern, north-eastern Atlantic and central Arctic Oceans. Values given in Tables 3 and 4 exclude episodes of significant anthropogenic influence. The aerosol in remote marine environment shows mostly unimodal growth with growth factors larger than in any other location. Observed growth factors are often between the growth factors of pure sulphate and pure sodium chloride particles (cp. Table 2), indicating internal or quasi-internal mixture of sulphate, sea salt and organics (see Sect. 3.2.3). Externally mixed sea salt dominated particles with growth factors larger than two are occasionally observed and are linked with high wind speeds on a regional scale with a critical value of the order of $10 \mathrm{~m} / \mathrm{s}$. They are more frequent at larger sizes but they are also found in the Aitken mode size range. Events of anthropogenic pollution were linked with decreasing growth factors of the dominant mode and with occurrence of an externally mixed marginally or moderately hygroscopic mode. Occasionally an externally mixed mode of moderately hygroscopic particles was reported in the absence of anthropogenic influence in the pack ice covered central Arctic Ocean during summer (Zhou et al., 2001) but the origin or composition of these particles remained unclear.

\subsubsection{CCN measurement}

Measurement of the cloud condensation nuclei, or CCN, provides a direct estimation of the propensity of aerosol to form cloud droplets. This propensity is reported as a potential cloud droplet concentration, realized at a specified water vapour supersaturation, or as a supersaturation-dependent spectrum of potential droplet concentrations. The latter is commonly referred to as the CCN activation spectrum. With the exception of the technique developed by Hudson (1989), CCN activation spectra are obtained from step-wise scans of the imposed supersaturation and presented as cumulative distributions. Activation spectra can also be derived from measurements of an aerosol size spectrum and composition, but a theoretical relationship between dry size and critical supersaturation (e.g. Fig. 2) is necessary for such calculations. Accordingly, CCN activation spectra (measured or derived) are classifiable as distribution-dependent properties (Sect. 3.2). Comparison of a measured activation spectrum with a derived spectrum constitutes a validation of the latter, provided that uncertainties in the measurements, as well as error introduced by simplifying assumptions (e.g. Köhler theory, mixing state, particle composition, etc.), are quantified. Such exercises establish reliable data sets for cloud model initialisation (e.g. Brenguier, 2003), and can also reveal limitations of the measurements (Chuang et al., 2000; Snider et al., 2003).

Over the past four decades several different $\mathrm{CCN}$ instruments have emerged, each with advantages and drawbacks inherent to their design. These designs are the isothermal haze chamber (Laktionov, 1972), the diffusion tube (Leaitch and Megaw, 1982), the CCN "remover" (Ji et al., 1998), 
the continuous-flow chamber (Sinnarwalla and Alofs, 1972; Chuang et al., 2000; Otto et al., 2002; Hudson, 1989; VanReken et al., 2003; Roberts and Nenes, 2005), the chemical diffusion chamber (Twomey, 1959), and the static thermal diffusion chamber (Squires and Twomey, 1966; Wieland, 1956; Lala and Jiusto, 1977; Oliveira and Vali, 1995; Giebl et al., 2002; Snider et al., 2003). Nenes et al. (2001a) has evaluated the performance of some of these instruments with a computational model. With the exception of the technique developed by (Hudson, 1989), CCN activation spectra are obtained from step-wise scans of the imposed supersaturation and presented as cumulative distributions. In the former technique the size distribution of activated droplets is measured and used to derive the differential $\mathrm{CCN}$ activation spectrum.

Since the CCN data are couched in terms of the maximum supersaturation experienced by particles within a $\mathrm{CCN}$ instrument (i.e. within the particle growth chamber), recent emphasis has been placed on accurate specification of this operationally-defined supersaturation. Seminal work on this problem was ambiguous showing either that the maximum supersaturation derived using chamber temperatures exceeded (Katz and Kocmond, 1973) or that it was consistent with the maximum supersaturation derived from a particle size sufficient for activation (Gerber et al., 1977; Alofs et al., 1979). Consistency in this context implies that measured temperatures, transformed to a maximum chamber supersaturation via a model of the temperature and vapour fields, is consistent with the chamber supersaturation derived by inputting the minimum dry size necessary for activation into Köhler theory. The laboratory work of Leaitch et al. (1999); Snider et al. (2003); Bilde et al. (2003); Bilde and Svenningsson (2004) reveal relationships of the form $S_{\text {eff }}=\kappa S_{\text {nom }}$ with $S_{\text {nom }}$ and $S_{\text {eff }}$ defined in terms of chamber temperature and particle size, respectively, and $\kappa$ varying between 0.8 and 0.5 . This correction is specific to measurements made with the University of Wyoming CCN instrument and is comparable to that made for other static diffusion instruments (Rissler et al., 2004). Since determination of $S_{\text {nom }}$ requires measurement and control of temperatures with an accuracy of better than $0.1{ }^{\circ} \mathrm{C}$, especially at supersaturations less than $0.2 \%$, the documented range of $\kappa$ is partially a reflection of the difficulty of conforming to the temperature measurement/control strictures. The value of $\kappa$ is also variable because of error propagating from the measurement of test particle size used in the determination of $S_{\text {eff }}$.

The duration of particle exposure to the effective maximum supersaturation is also a parameter which deserves consideration in an evaluation of $\mathrm{CCN}$ data, or in their use in closure studies. Residence time in the $\mathrm{CCN}$ instrument is especially important if kinetic control of water uptake is amplified by particles with coatings that produce low or varied water accommodation coefficients (see Sect. 4.1). Ideally this time interval should mimic that which occurs during activation in an updraft. According to parcel model calculations (cf. Rogers and Yau, 1989), the duration of the peak supersaturation varies with vertical velocity and aerosol background. A representative value is $\sim 10 \mathrm{~s}$ and is consistent with growth times in many $\mathrm{CCN}$ instruments. Although speculation does exist that a mismatch of the instrument-imposed and natural times can lead to bias in the estimation of $\mathrm{CCN}$ activation (Chuang et al., 1997), this issue has not been addressed experimentally. Investigations planned for a large continuousflow instrument (Stratmann et al., 2004) may contribute to resolution of this issue.

\subsubsection{Aerosol mixing-state with respect to water uptake}

A complicated composition mixing state is expected in the ambient atmosphere due to the various primary particle sources and numerous processes altering particle composition. Each kind of internally mixed particle may contain a range of fractional contributions of each constituent, hereinafter referred to as quasi-internal mixtures. External mixing of differently hygroscopic compounds results in distinct growth modes, whereas quasi-internal mixing results in spread of observed growth factors around the growth factor corresponding to the mean composition. This description is consistent with the discussion of composition presented in Sect. 3.1.4.2.1. Detection of different $G F_{D}$ modes or a continuous $G F_{D}$ distribution with a HTDMA implies that the aerosol is to some extent externally mixed. However, detection of a single growth factor does not exclude the presence of externally mixed particles, as different mixtures may still have similar growth factors. At least two distinct growth factor modes are frequently observed in various environments, showing that external mixing is widespread. Some studies even report three to six simultaneous growth modes (Berg et al., 1998a; Cocker et al., 2001; Carrico et al., 2005). In both Aitken and accumulation mode size ranges, an externally mixed mode of marginally hygroscopic particles $\left(G F_{D i} 1.15\right.$ at $\left.90 \% R H\right)$ is frequently found in most environments. Numerous studies combining different methods indicate that these marginally hygroscopic particles are dominated by elemental carbon (Covert and Heintzenberg, 1984; Heintzenberg and Covert, 1987; Zhang et al., 1993; Maßling et al., 2003) and/or mineral dust (Zhang et al., 1993; Dick et al., 1998, 2000; Weingartner et al., 2001), as can be expected from the hygroscopic behaviour of pure substances (Table 2). Mineral dust appears to be found in particles with small growth factors only, whereas elemental carbon is also found in particles with larger growth factors. Small amounts of inorganic salts or organic compounds may also be present in these marginally hygroscopic particles being responsible for small water uptake. Though marginally hygroscopic particles are nearly ubiquitous, the dominating growth mode in most environments is readily hygroscopic with $G F_{D}$ values ranging from about 1.2 to 1.8 at $90 \% R H$. Inorganic salts and organic compounds are understood to be the main ingredients of these, quasi-internally mixed, readily hygroscopic 
particles. Organic compounds or inorganic salts may dominate depending on air mass and location, resulting in moderate or large hygroscopic growth factors, respectively (cp. Table 2).

Aerosol mixing state with respect to hygroscopic growth is likely to be an important parameter determining the properties of resulting clouds via its effect on critical supersaturation, Eq. (5). The absolute value of hygroscopicity at a given particle size will determine the critical supersaturation for cloud activation, directly influencing the water activity in Eq. (1). The mixing state of particles around the threshold dry size, on the other hand, may determine the number of particles of a given size which will activate, thus potentially affecting the number of cloud droplets. However, while there are studies investigating the effect of external mixtures of particles with two discrete growth factors (e.g. Kulmala et al., 1996), we are not aware of studies investigating the importance of growth spread due to quasi-internal mixing. Regardless, the effect of the $G F_{D}$ on the number of cloud droplets is difficult to generalise since it depends on the dynamical interplay between aerosol size distribution and updraught.

Values for growth spread are commonly reported as relative growth spread or diameter dispersion factor $S_{D}$, defined such that the standard deviation of a growth mode $\Delta G F_{D}=S_{D} \cdot G F_{D}$. A spread of $S_{D}=5 \%$ at a growth factor of $G F_{D}=1.5$ means that $34 \%$ of particles have a growth factors smaller than 1.425 or larger than 1.575. Literature values of growth spread are scarce due to the limited resolution of HTDMA inversion algorithms. Nevertheless there are studies in urban and polluted continental environments reporting significant growth spread of 5\% for both Aitken and accumulation mode size ranges and both dominant and second growth mode, showing that quasi-internally mixed particles and external mixtures of different kind of quasi-internally mixtures are frequent phenomena (McMurry and Stolzenburg, 1989; Zhang et al., 1993; Svenningsson et al., 1994, 1997). Aklilu and Mozurkewich (2004) reported clear relationship between spread and growth factor with low spread at smallest and largest growth factors, and spread as high as $11 \%$ at intermediate growth factors. This result indicates nearly internal mixture in the extreme cases and quasi-internal mixture in balanced cases where both more and less hygroscopic compounds where present in similar amounts. On the other hand, more hygroscopic growth modes with hardly any spread have also been reported in remote Arctic and high-alpine environments indicating that atmospheric ageing tends to transform the background aerosol into an internal mixture (Covert and Heintzenberg, 1993; Weingartner et al., 2002).

\section{Extensions of theoretical activation treatments ac- counting for aerosol complexity}

From the fundamental and derived properties of ambient atmospheric aerosol particles presented in Sect. 3 it is evident that their complexity will not be fully reflected by using simplifying assumptions in the simple activation and growth treatments described in Sects. 2 and 3.1. This section describes treatments and theoretical modifications required to begin to address some of the more important factors raised by the aerosol complexity.

\subsection{The effect of component solubility and surface activity on droplet activation and growth}

Beside chemical species that dissolve easily into water, atmospheric aerosol particles contain numerous substances which are partially or completely insoluble. In the simplified form of the Köhler Eq. (2), it is assumed that the aqueous solution droplets contain neither solid inclusions nor immiscible liquid phases. Components that do not readily pass into aqueous solution have been postulated to have a number of effects on droplet activation and growth which have been treated using various assumptions and simplifications. They can broadly be categorised into i) suppression of the number of solute molecules in solution in a droplet of a given size, ii) reduction of surface tension of an activating droplet and iii) inhibition of the uptake of water. This section presents a discussion of the range of theoretical treatments, hypothesised effects and evidence from field and laboratory measurements. A discussion of the solubility of surface active components in real aerosol is presented and the implications on the validity of theoretical treatments are evaluated.

\subsubsection{Insoluble solid components}

Hänel (1976) presented a simple correction which accounts for the presence of insoluble substances in the droplets assuming that the insoluble core is fully immersed into the aqueous solution. Gorbunov and Hamilton (1997) and Gorbunov et al. (1998) extended the consideration into systems in which the insoluble core is only partially covered by the water solution. In Köhler theory, such a situation can be described with a modified Kelvin term that depends in rather complex manner of the radii of the solid particle (assumed spherical) and the attached aqueous solution droplet, and of the contact angle between the two.

\subsubsection{Largely insoluble solid or immiscible liquid phases}

The above approach is only possible for an assumed spherical insoluble inclusion. This is a much idealised description of a special case of the separation of immiscible phases. The formation of solid and mobile films is an equally idealised situation, though mobile amphiphilic components may preferentially adopt such surface partitioning (see Sects. 4.1.4-4.1.8). 
Clegg et al. (2001) presented evidence based on laboratory data and thermodynamic calculations for the formation of immiscible phases even in simple aqueous salt/organic solutions over certain solute concentration ranges; there is no reason to believe that condensed organic non-polar molecules will pass into aqueous solution in atmospheric aerosol and cloud droplets when there may be a more thermodynamically favourable phase distribution.

\subsubsection{Components of limited solubility}

The effect of slightly soluble substances on droplet activation was first treated by Shulman et al. (1996). As the $R H$ increases and the droplet takes up more water, slightly soluble substances dissolve gradually. Thus, the amount of solute in the droplet increases and the size of a slightly soluble solid inclusion will decrease with increasing $R H$ until it dissolves completely. A modified Köhler equation accounting for such behaviour can be given as (Laaksonen et al., 1998):

$S=\frac{e}{e_{s}} \approx 1+\frac{A}{r}-\frac{B}{r^{3}-r_{s s}^{3}}-\gamma_{s s}$

where $r_{s s}$ is the radius of the (assumedly spherical) undissolved core of the slightly soluble substance and $\gamma_{s s}$ is given by

$\gamma_{s s}=\frac{M_{w}}{M_{s s}} v_{s s} \Gamma, \quad \quad r_{s s}>0$

$\gamma_{s s}=\frac{v_{s s} \Gamma M_{w} m_{w}^{0}}{M_{s s} m_{w}}, \quad r_{s s}=0$

Here $\Gamma$ denotes the solubility of the slightly soluble substance and $m_{w}^{0}$ is the mass of water needed to dissolve the slightly soluble substance completely.

Shulman et al. (1996) showed that the presence of slightly soluble material in the $\mathrm{CCN}$ leads to alteration of the Köhler curve shape: instead of a single maximum, two maxima appear, separated by a cusp at the point where the slightly soluble compound is completely dissolved. Depending on the value of $\gamma$ and the relative amounts of the soluble and slightly soluble substances, the cusp can appear on either side of the global maximum of the curve. Shulman et al. (1996) speculated that for a particle containing several slightly soluble substances, there could be a series of cusps in the Köhler curve, leading to overall flattening of the curve and changes in the activation behaviour of the particle. In any case, Köhler theory predicts that the effect of the limited solubility is to increase critical supersaturation relative to the unlimited solubility case. The maximum effect of course occurs when the solubility approaches zero. This approach takes no account of the effect of the undissolved fraction of the slightly soluble component on activation such as described in Sect. 4.1.1 or Sect. 4.1.2 owing to the assumption that it is solid, spherical and completely immersed.

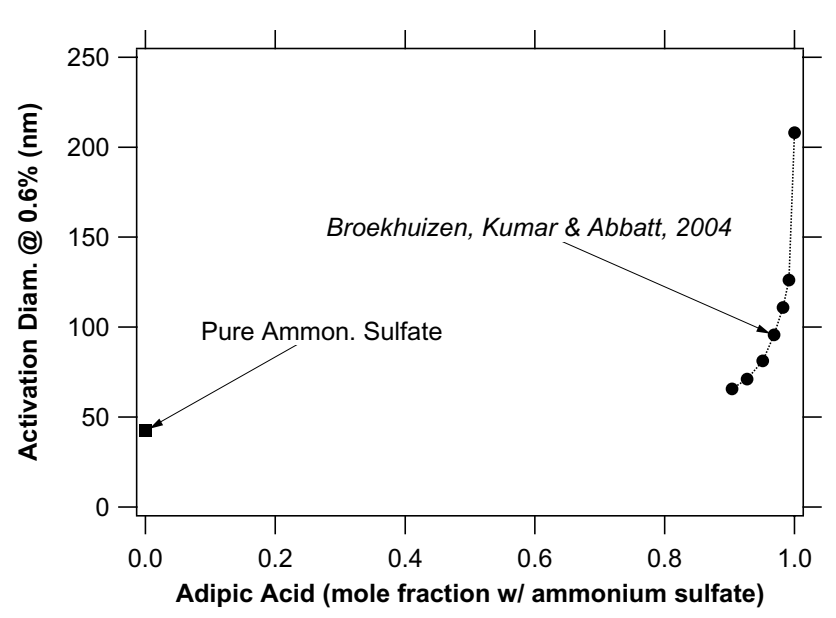

Fig. 9. CCN Activation diameter of particles at $0.6 \%$ as a function of their adipic acid dry mole fraction. Note that a very high proportion of the particle must comprise the organic component before the activation diameter is significantly increased.

Broekhuizen et al. (2004), Bilde and Svenningsson (2004) and Henning et al. (2005) all studied aspects of the effects of slightly soluble substances on CCN activation. All studies found that initial particle phase played a significant role in the critical supersaturation of particles containing only slightly soluble substances. If the particle was initially totally liquid the critical supersaturation was found to be lower. Bilde and Svenningsson (2004) postulated that an increased critical supersaturation was due to to an undissolved core forming an activation barrier when the growing particle is smaller than the fully dissolved wet size. The activation barrier led to a critical supersaturation greater than the local maximum equilibrium supersaturation which would have otherwise been the critical value. Whilst the $R H$ history (in terms of predicted deliquescence/efflorescence relative humidities) of a particle in the atmosphere may be such that solid phases should exist for single organic components in solution, Marcolli et al. (2004) recently demonstrated that the multicomponent organic solutions favoured the liquid phase. For complex mixtures found in the atmosphere, it may be expected that aerosol comprising slightly soluble organics would be unlikely to contain undissolved material. In addition, as illustrated by Fig. 9, only a very small amount of soluble inorganic salt is required to reduce the critical supersaturation and significantly enhance activation. On balance, it may be considered that the effect of limited solubility is of limited relevance to real multicomponent atmospheric aerosol activation.

\subsubsection{An introduction to the effect of surface active com- ponents}

It is clear from all forms of the Köhler equation that any component of an activating particle which can significantly alter 
the surface tension may influence its activation behaviour. All other things being equal, a reduction in surface tension will lead to a reduction in the Kelvin term, hence the critical supersaturation, leading to possible effects on cloud formation (Shulman et al., 1996; Li et al., 1998; Facchini et al., 1999a; Charlson et al., 2001; Mircea et al., 2002; Nenes et al., 2002; Feingold and Chuang, 2002; Lohmann et al., 2004). The surface tension of pure water is $72.8 \mathrm{mN} / \mathrm{m}$ at $20^{\circ} \mathrm{C}$ and hydrogen bonding in water leads to a much greater cohesion between molecules at the surface than exhibited by less polar liquids and so water has a higher surface tension than organic liquids. Compounds such as soaps and detergents which disrupt the surface cohesion and reduce the surface tension may be termed surface active. The term surfactant is frequently used to describe linear amphiphilic molecules with a polar hydrophilic "head" group and a hydrophobic "tail". They are typically sparingly soluble in both organic solvents and water and reduce the surface tension of water by adsorbing at the air-water interface. Care should be taken in the interpretation of the definition of surfactant; we shall not limit the definition to such compounds, but shall use surfactant with its broad definition as a general term for any surface active agent. The importance of surfactants in aerosol and cloud water has been recognized for many years (Graedel and Weschler, 1981; Gill et al., 1983; Seidl and Hänel, 1983) but only recently the suggestion has been put forward that surface active organic species can have significant effects on global climate, pollution and human health (Brimblecombe and Latif, 2004).

The range, variable nature and concentration of surface active components which may be present in aerosol particles determine the behaviour of a surfactant mixture at the cloud droplet/air interface. Therefore, knowledge of these properties is a prerequisite for realistically describing the surface tension in a growing droplet. For example, a more sparingly soluble surfactant will reduce the surface tension whilst partitioning towards the surface. This will reduce the surface tension and the Kelvin term, but may also reduce the effective number of solute molecules in bulk solution, reducing the Raoult term. More soluble compounds will have less of an effect on the Raoult term. The degree to which a surfactant partitions between the bulk and the surface of the droplet (see Sects. 4.1.7 and 4.1.8) is dependent on the nature of the dominant surface active components (Sect. 4.1.9).

\subsubsection{The existence of insoluble surface films}

As water solubility decreases there will be an increasing tendency towards phase separation. A special case of incomplete phase separation will be exhibited by amphiphilic surfactant molecules, which, under specific conditions, may form organised organic layers on aqueous surfaces. The classical examples of such compounds are long-chain fatty acids but very many natural compounds exhibit amphiphilic character (http://www.cyberlipid.org/index.htm). Very little or- ganic material would be required to coat an aerosol particle and both online and offline composition measurements suggest that many particles contain sufficient organic mass; unfortunately, most measurement techniques cannot discern whether the organics are at the surface.

The concept of an inverted micelle with a structure similar to a cell membrane has stimulated significant research over the last two decades. Early laboratory studies and the expected effects of organic films on aqueous atmospheric systems were summarised by Gill et al. (1983) in a comprehensive overview. Consideration of thermodynamic and dynamic boundary conditions for film formation in atmosphere led them to the conclusion that if film formation takes place, it is most likely on submicron aerosol particles. The two dimensional nature of films necessitates their phase description as solid, liquid-condensed, liquid-expanded or gaseous dependent on their molecular packing. Transition between phases is not instantaneous or even, with domains of different phases coexisting, depending on the area available on the surface for the molecules. Gill et al. were concerned with condensed and expanded films, the first tight, with a wellordered packing structure, the second loose, unordered, possibly with pores, or more liquid like. Both types of films may alter the condensed phase chemistry in particles. Because of packing arguments, the formation of closed, condensed films is intrinsically difficult on the curved surfaces of submicron atmospheric particles, even if they would normally form on flat surfaces. Moreover, in case of atmospheric systems, mixtures of compounds at the surface reduce the possibility of the formation of large well-ordered domains. Thus, on particles in the atmosphere, expanded films are more likely. Seidl (2000) demonstrated by model considerations, that the concentration of film forming compounds in most areas is so small that closed films are not expected. On the other hand, compounds which would per se not be able to form closed domains, might be able do that in mixtures with others molecules which are able to fill the gaps - indeed, this is the basis behind the methodology of co-spreading of thin films in laboratory. For example, Gilman et al. (2004) demonstrated that stearic acid can stabilize incorporation of nonamphiphilic octadecane in organic films on the several hour time scale, indicating that non-polar layers may be formed on top of an amphiphilic basis.

The direct evidence for coated aqueous particles in the atmosphere is difficult to obtain and scarce. In order to form stable films, compounds need to have a low vapour pressure; indeed, if the hydrophobic chain is sufficiently long to keep the compound largely on the surface and out of solution, the vapour pressure will inevitably be low. This reduces possible pathways in the atmosphere. Either the film-forming compounds are emitted in primary particles or are formed via (multiple) oxidation of larger (unsaturated) hydrocarbons. After the primary oxidation step in the gas phase the subsequent oxidation would need to take place in the particulate phase as it would be unlikely that oxidation products would 
have sufficient vapour pressure to sustain high gas-phase concentrations. Moreover, oligomerisation reactions taking place in the particulate phase (Gao et al., 2004; Kalberer et al., 2004; Tolocka et al., 2004) may lengthen the carbon chain, decreasing the aqueous solubility; thus the shorterchain monomer units may originally partition from the gas phase. Long chain fatty acids from cellular debris are found on top of the ocean surface, and it can be expected that the fatty acids become part of the marine aerosol by sea spray generation mechanisms. It has been postulated that the fatty acids form an inverted micelle around the brine core (Ellison et al., 1999). Two recent studies (Mochida et al., 2002; Tervahattu et al., 2005) have reported evidence strongly supporting the existence of layers of fatty acids on marine aerosols. Folkers et al. (2003) demonstrated in chamber experiments, that ozonolysis products of a-pinene are forming films on aqueous ammonium bisulfate particles. The films retard the heterogeneous $\mathrm{N}_{2} \mathrm{O}_{5}$ hydrolysis on time scales of several tens of minutes, thus changed the surface water budget of the seed particles. Similar effects were achieved by adding ozone to outside air in the presence of aqueous sulphate and bisulphate seed particles, with a corresponding decrease of monoterpenes as monitored by GC-MS.

If films are present, the organic material is exposed at the interface to the oxidative gas-phase and they may be the preferred location for condensed phase oxidation (Moise and Rudich, 2002; Broekhuizen et al., 2004; Katrib et al., 2004 ) or the recently discussed oligomerisation reactions (Gao et al., 2004; Kalberer et al., 2004; Tolocka et al., 2004). The first would tend to enhance the solubility of the film forming material (Ellison et al., 1999), thus enhancing the overall water soluble organic fraction (WSOC). The latter could stabilize and tighten the film, by converting expanded into condensed films. Moreover, oligomerisation reactions may increase the water insoluble organic fraction (WINSOC) and HULIS fractions. Organic films could therefore be an important medium for chemical aging of atmospheric particles affecting hygroscopic and CCN properties slowly during their lifetime.

4.1.6 Films as a barrier to gaseous transfer and their effects on droplet growth

A significant inhibition of vapour transfer, e.g. of water from the gas into the particulate phase, is expected only for the condensed films. Even then, films of the order of several $10 \mathrm{~nm}$ constitute a simple diffusional transport barrier only on sub-second time scale. This can easily estimated from the $x_{\text {rms }}$ for Brownian motion:

$\tau=\frac{x_{\mathrm{rms}}^{2}}{D}$

For diffusion coefficients $D$ between $10^{-5} \mathrm{~cm}^{2} / \mathrm{s}$ (liquid phase diffusion) and $10^{-9} \mathrm{~cm}^{2} / \mathrm{s}$ (solid phase and slow polymer diffusion) and a layer thickness $x_{\mathrm{rms}}=50 \mathrm{~nm}$, the char- acteristic $\tau$ ranges between $2.5 \mu$ s and $250 \mathrm{~ms}$. Two possible reasons exist for the retardation of mass transfer of water through films of nm thickness: a strongly reduced surface accommodation coefficient $\alpha$ of water on the nonpolar surfaces of the organic coatings or a very small partitioning coefficient of water in the hydrophobic parts of the film forming material. Accommodation coefficients of water on condensed films systems are in the range $1 \times 10^{-3}$ to $1 \times 10^{-5}$ (Chuang, 2003 and references therein). In Mexico City Chuang (2003) made measurements consistent with some particles having greatly reduced (order $10^{-5}$ ) accommodation coefficient for water. However, fewer than $2 \%$ of the particles were in this category and they are probably even rarer in remote regions and the free troposphere.

In the laboratory studies of Cruz and Pandis (1998) solid ammonium sulphate particle were coated with glutaric acid (water soluble) and dioctylphthalate DOP (water insoluble oil). The activation to $\mathrm{CCN}$ was measured at $0.5 \%$ supersaturation. The ammonium sulphate/glutaric acid particles behaved as expected from Köhler theory, the DOP films even at $70 \%$ mass fraction of DOP had no influence. Raymond and Pandis (2003) studied CCN activation of ammonium sulphate covered with layers of hexadecane of different thickness at supersaturations of $0.3 \%$ and $1 \%$. The coated particles behaved like pure ammonium sulphate particles within the errors of the measurements. However, Raymond and Pandis (2003) conceded the possibility that the films were not closed. Although such studies indicate that organic coatings may not prevent activation, they have been shown to slow the kinetics of water uptake, such that deviation from the equilibrium can be observed over the time span of the probing experiments (Barnes, 1986, in Chuang, 2003). Xiong et al. (1998) studied the hygroscopic growth of sulphuric acid aerosols $(40-120 \mathrm{~nm})$ with and without films of long chain fatty acids on 6-10s time scale. After coating the particles with mono- or multilayer films of lauric or stearic acid, they observed a decreasing growth rate with increasing layer thickness from $10 \%$ to $20 \%$ compared to pure sulphuric acid droplets. For oleic acid such an effect could not be found for a monolayer and minor effects were observed for the multilayer case, likely resulting from the double bond of oleic acid leading to less efficient packing structure of the layer. In the atmosphere where it is unlikely that a single surfactant compound is present, the packing will very likely be at least as disturbed. Atmospheric organic compounds forming expanded film coatings are not expected to affect the equilibration timescales nor the equilibrium composition of the aqueous core. This is supported by the applicability of simple additive mixing rules for inorganic and organic growth behaviour in certain systems (Cruz and Pandis, 1998; Saathoff et al., 2003), though care must be taken since inorganic/organic solute interactions in some systems are nonzero and may have solvent or antisolvent effects (see e.g. Topping et al., 2005b, for a discussion) and it is not entirely clear that additivity should be applicable. 
From the results above it is clear that if at all, only tightly, condensed ordered films or thick hydrophobic layers can retard water uptake on several tens of second and larger time scales. Though difficult to form in the atmosphere, it is possible that such films may be found in the marine influenced atmosphere and possibly in populated urban regions (Seidl, 2000; Tervahattu et al., 2005). The exact mechanism of retardation has not been experimentally proven, but it may be expected that "non-wettability" i.e. a strongly reduced mass accommodation coefficient $\alpha$ of water on hydrophobic surfaces and also a strongly reduced water solubility in the hydrophobic part of the organic layers are the reason for the retardation effects. In any case, expanded films are probably found more often on tropospheric aerosols and are unlikely to influence the hygroscopic or $\mathrm{CCN}$ properties greatly by mass transfer limitation.

\subsubsection{The effects of surface partitioning on activation}

Insoluble film formation is an extreme example of the partitioning of organic material and aqueous "substrate". Surface active compounds, by definition, tend to be more concentrated in the surface layer of aqueous solutions than in the bulk of the solution. Surface tension reduction is related to the surface concentration, but in practice experimental surface tensions are always given as a function of the bulk solution concentration. For large systems, the bulk concentration can be calculated from the total moles of the substances involved as the amount of surfactant in the surface layer is a negligible fraction of its total amount in the system. However, for small systems with a high surface-to-volume ratio the situation is different, and depending on the degree of surface activity of the component, the partitioning of the surfactant between the bulk and the surface has to be accounted for when calculating the bulk concentration from given total numbers of moles. Bianco and Marmur (1992) derived equations for this purpose starting from mass conservation, the Gibbs adsorption equation, and the Szyszkowski equation which relates the solution surface tension to the bulk concentration of solute. They showed that with aqueous SDS (sodium dodecyl sulfate) droplets, the bulk concentration differs from that calculated simply from the total number of moles when the droplets are smaller than about $20 \mu \mathrm{m}$ in diameter. Laaksonen (1993) presented a slightly less approximate treatment and showed that for a weaker surfactant with a smaller molar volume (n-propanol), the surface-tovolume partitioning affects bulk concentrations of droplets smaller than roughly half a micron. This indicates that for micron sized cloud droplets, the bulk concentration of the solute can be estimated from the total number of solute moles for species which do not depress the surface tension of water more efficiently than alcohols and which have relatively small molecular volumes. Such species would include e.g. carboxylic acids.
The studies of Bianco and Marmur (1992) and Laaksonen (1993) focused on the properties of surfactant solution droplets, but they did not consider the equilibrium with the ambient water vapour. This problem was first treated by $\mathrm{Li}$ et al. (1998) who noted that the surfactant partitioning has to be taken into account when the Kelvin effect is calculated for droplets containing surfactant. For droplets with known total numbers of water and surfactant, the bulk molar concentration of surfactant, $C_{\mathrm{sft}}$, is obtained from:

$C_{\mathrm{sft}}=\frac{m_{\mathrm{sft}} / M_{\mathrm{sft}}-4 \pi r^{2} \Gamma_{\mathrm{sft}}}{4 / 3 \pi r^{3}}$

where $m_{\mathrm{sft}}$ and $M_{\mathrm{sft}}$ are the dry mass and the molecular weight of the surfactant, respectively, and $\Gamma_{s}$ is the surface excess concentration of the surfactant, which is given by the Gibbs adsorption equation as

$\Gamma_{s}=-\frac{1}{\omega R T} \frac{\delta \sigma}{\delta \ln C_{\mathrm{sft}}}$

Here, the parameter $\omega$ denotes the number of ions the surfactant dissociates into, except for systems containing additional dissociable materials with common ions with the surfactant. E.g. Li et al. (1998) considered dry particles containing SDS and sodium chloride; in that case: $\omega=1+C_{\mathrm{sft}} /\left(C_{\mathrm{sft}}+C_{s}\right)$.

The conclusion of $\mathrm{Li}$ et al. (1998) was that the reduction in surface tension caused by pure SDS particles reduces the critical supersaturation with respect to that calculated using Eq. (1); however, with NaCl-SDS particles, the surface tension effect is dominated by the fact that for a solute particle of a fixed size, replacement of small $\mathrm{NaCl}$ molecules by much larger SDS molecules causes a reduction in the Raoult term, and thereby an increase in the critical supersaturation. In a commentary by Facchini et al. (2001), it was argued that the result of Li et al. (1998) is due to the choice of the specific chemical composition, and that for mixtures of inorganic salts and organic surfactants more representative of atmospheric aerosols than SDS, the presence of the surfactants may in fact lead to decreased $S_{c}$. In their reply, Rood and Williams (2001) argued that the results of Facchini et al. (2001) are sensitive to uncertainties in the values of molecular parameters such as molecular weight, the van't Hoff factor, and the density of the assumed surfactants.

The Raoult term evaluated by $\mathrm{Li}$ et al. (1998) was calculated using the total number of surfactant molecules in the droplet. Sorjamaa et al. (2004) noted that as with experimental surface tension, the vapour pressure reduction of water due to solutes is always given as a function of bulk solute concentration, and therefore surface-to volume partitioning of the surfactant should be accounted for also when the Raoult term is determined. The approximations made by Sorjamaa et al. (2004) are slightly different from those of $\mathrm{Li}$ et al. (1998), but the differences should be noticeable only for droplets smaller than about $10 \mathrm{~nm}$ in diameter. When 
accounting for the surfactant partitioning for a mixed saltsurfactant particle, the Raoult term becomes:

$B=\frac{M_{w}}{\left(4 / 3 \pi \rho_{w}\right)}\left[\frac{v_{s} m_{s}}{M_{s}}+4 / 3 \pi r^{3} \rho_{\mathrm{sft}} C_{\mathrm{sft}}\right]$

The Köhler curves calculated by Sorjamaa et al. (2004) for SDS-NaCl particles show that when the surfactant partitioning is also accounted for in the Raoult term, the critical supersaturations are further increased. In certain cases, (e.g. 50/50 mass fraction), the $S_{c}$ can even become higher than that calculated using the surface tension of pure water and without accounting for the partitioning. Figure 10 shows a comparison of critical supersaturations as a function of dry particle radius for 30/70\% NaCl-SDS particles obtained using the different approaches.

The theoretical considerations of the role of surfactant partitioning are backed up by the experimental results of Sorjamaa et al. (2004). They measured, using a CCN counter, critical supersaturations for pure SDS particles. Their results were close to the experimental results presented by Rood and Williams (2001), and in excellent agreement with their own theoretical predictions. The initial measurements for $\mathrm{NaCl}-$ SDS particle critical supersaturations presented by Rood and Williams (2001), on the other hand, seem to corroborate the theory of $\mathrm{Li}$ et al. (1998). More work needs to be carried out to clarify the details; however, it seems clear that, when soluble compounds with the surface active nature similar to SDS are considered, the surfactant partitioning needs to be accounted for in cloud modelling and Köhler theory studies, and simply accounting for surface tension reduction by calculating the surfactant concentration from its total mass in the droplet leads to underestimation of the critical supersaturation.

With compounds of limited solubility, the surfactant partitioning has somewhat different consequences than with completely soluble species. Sorjamaa et al. (2004) showed, making calculations for the pinonic acid-ammonium sulfate system, that up to the point of complete dissolution of the slightly soluble compound, the surface tension reduction is similar as when calculated without accounting for the partitioning. This is because, as long as a solid inclusion of the slightly soluble substance exists, its bulk mole fraction is simply determined by its solubility. The main difference between the two approaches is now that the slightly soluble substance is consumed more rapidly when the partitioning to the surface is accounted for - at constant bulk mole fraction, a larger amount of material must be dissolved in order to account for the concentrated layer of surfactant at the droplet surface. Thus, the cusp at the Köhler curve appears at a smaller droplet radius. Concerning activation, then, the surfactant partitioning consideration is important only in cases in which the cusp appears at smaller droplet sizes than the global maximum of the Köhler curve.

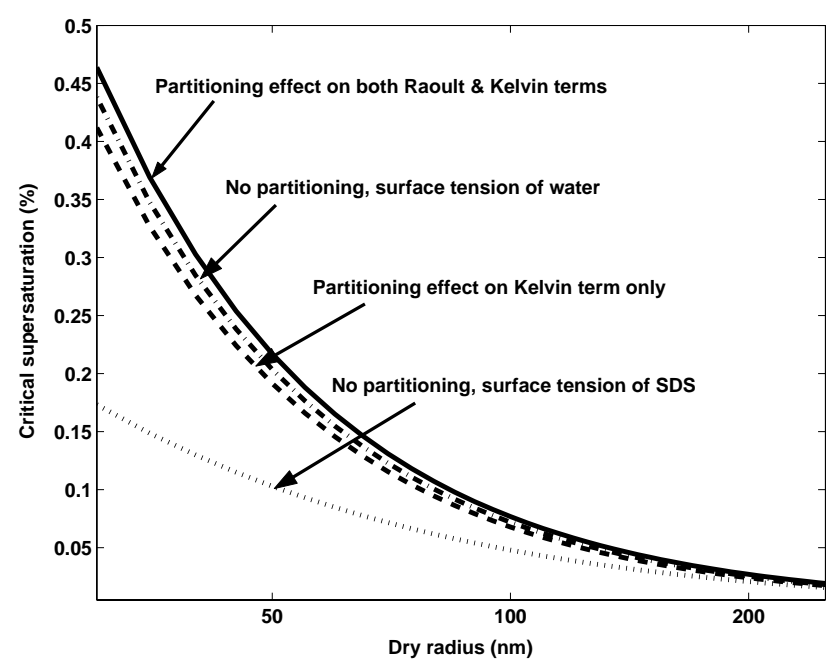

Fig. 10. Critical supersaturation plotted against dry particle radius for $30 / 70 \%$ (by mass) NaCl-SDS particles.

4.1.8 A simplified consideration of the effect of filmforming compounds on droplet activation

As discussed in Sect. 4.1.4, there are certain conditions in which it may be envisaged that films of essentially-insoluble amphiphilic molecules are present on the surface of growing cloud droplets. It is possible to consider the effect that the surface tension reduction of such films would have on the critical supersaturation and radius in a relatively straightforward manner. The characteristic surface pressure-area $(\pi-A)$ isotherm across all surface phases may be used to estimate the surface tension of the growing droplet as a function of its surface area and molecular coverage. Assuming complete surface partitioning due to the negligible solubility of stearic acid (no contribution to the Raoult effect from stearate ions) Fig. 11 shows the change in the Köhler curves on replacement of 0.3 solute mass fraction of $\left(\mathrm{NH}_{4}\right)_{2} \mathrm{SO}_{4}$ by stearic acid in $\left(\mathrm{NH}_{4}\right)_{2} \mathrm{SO}_{4}$ droplets with the $\mathrm{C}_{18}$ fatty acid forming a film on the surface.

It can be seen that, although an insoluble film will reduce the surface tension, its effect will be to increase $S_{c}$ and reduce the number of activated droplets (by replacement of solute with a van't Hoff factor of 3 with a single ion in solution in the Raoult effect). It must be clarified whether ordered organic films truly do exist on growing cloud droplets as postulated in Sect. 4.1.5, whether surface partitioning effects such as exhibited by SDS as discussed in Sect. 4.1.7 are significant or whether surface tension suppression predominantly results from WSOC as contended in the next Sect. 4.1.9. 


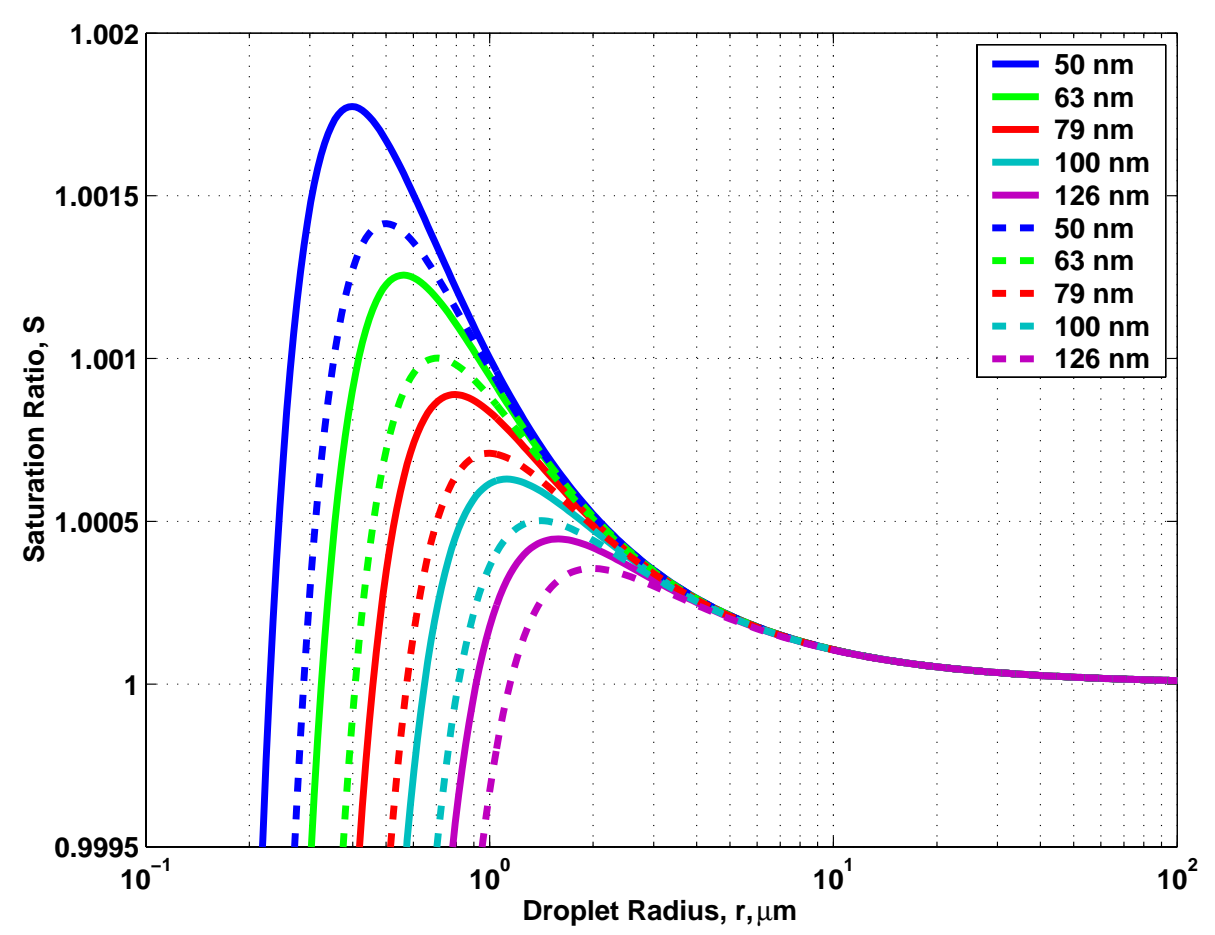

Fig. 11. Köhler curves calculated using Eq. (2) with surface tension reduction calculated using the $\pi-A$ isotherm for stearic acid with $30 \%$ mass fraction solute stearic acid and $70 \%\left(\mathrm{NH}_{4}\right)_{2} \mathrm{SO}_{4}$. Solid curves include surfactant, dashed are pure $\left(\mathrm{NH}_{4}\right)_{2} \mathrm{SO}_{4}$.

4.1.9 Soluble surfactants and parameterised surface tension of multicomponent aerosol based on field and laboratory measurements

Surface tension measurements of fog, cloud samples and aerosol water extracts are still very sparse (Seidl, 2000 and references therein; Facchini et al., 2000; Hitzenberger et al., 2002; O'Dowd et al., 2004; Decesari et al., 2005; Kiss et al., 2005); for this reason, and due to the lack of a complete chemical characterisation of the organic matter, the effect of surfactants cannot be assessed at the global level. Several studies have become available on the surface active effects of the organic components in atmospheric aerosol. Recent investigation of the dynamic surfactant behaviour in aerosol and cloud samples from different environments by Decesari et al. (2003) concluded that water soluble organic species are mainly responsible for lowering the surface tension of atmospheric aqueous solutions. It has also been postulated that humic-like substances (HULIS) are the main class of surface active compounds in atmospheric aerosol from different environments, while mono- and dicarboxylic acids and other non acidic compounds contribute only to a lesser extent (Facchini et al., 2000). Kiss et al. (2005) have further shown that the surface active properties of the HULIS contained in the aerosol are different from standards of terrestrial and aquatic humic compounds. Several papers have recently suggested that marine aerosol contains organic surfactants both soluble and insoluble in water, whose concentration is enhanced during high biological productivity periods and which may have a significant effect on the physico-chemical properties of the particles (Ellison et al., 1999; Mochida et al., 2002; Tervahattu et al., 2005; Cavalli et al., 2004b, and references therein).

For water soluble surface active material, Decesari et al. (2003) contend that the empirical Syszkowski-Langmuir equation can be used to describe the dependence of $\sigma$ on organic carbon concentration:

$\sigma_{s}=\sigma_{0}-b T \ln (1+a C)$

where $\sigma_{0}$ is the surface tension of pure water in $\mathrm{mN} / \mathrm{m}, C$ is the concentration of soluble carbon in moles ${ }^{-1}, T$ is the temperature in $K$, and $b$ and $a$ are empirical constants fitted to experimental data. The values of $a$ and $b$ depend on the specific chemical composition of WSOC in aerosol. Moreover, the value of the surface tension depends on the amount of soluble carbon, $C$, which is determined by the mass and solubility of the species, and thus, also depends on the WSOC chemical composition.

Figure 12 shows the surface tension as a function of dissolved organic carbon concentration computed with Eq. (14) and by using $a$ and $b$ parameters reported in Table 5 for: Po Valley fog samples (Facchini et al., 1999a), Tenerife cloud samples, Mace Head aerosol water extracts (O'Dowd et al., 2004; Cavalli et al., 2004b), biomass burning aerosol water extracts (Decesari et al., 2006) and ACE-Asia cloud water samples (Decesari et al., 2005). The different curves 
Table 5. Parameters for Eq. (14) (Syszkowski-Langmuir equation) that describes surface tension behaviour of fog, cloud and aerosol water extract samples from different environments. The fourth and fifth columns show the percent changes in critical supersaturation $\left(S_{c}\right)$ and critical diameter $\left(D_{c}\right)$ calculated using the parameterizations of surface tension for each environment $\left(\left(s_{S T}, D_{S T}\right)\right.$ and surface tension of pure water $\left(s_{\mathrm{H}_{2} \mathrm{O}}, \mathrm{D}_{\mathrm{H}_{2} \mathrm{O}}\right)$ ). The calculations were made for an aerosol particle of $0.05 \mathrm{~m}$ dry diameter and the chemical composition was considered constant for the different cases (70\% soluble: $40 \%$ inorganic salts and $30 \%$ organic species) Note that $\sigma_{0}$ is the surface tension of pure water at the temperature at which the measurements were performed and parameters $b$ and $a$ were fitted to the experimental data.

\begin{tabular}{lccccc}
\hline & $\sigma_{0}\left(\mathrm{mNm}^{-1}\right)$ & $b\left(\mathrm{mNm}^{-1} \mathrm{~K}^{-1}\right)$ & $a(\mathrm{lmole}-1)$ & $\left(S_{\left.S_{T}--S_{\mathrm{H}_{2} \mathrm{O}}\right) /}\right.$ & $\begin{array}{c}\left(D_{S T}--D_{\mathrm{H}_{2} \mathrm{O}}\right) / \\
D_{\mathrm{H}_{2} \mathrm{O}}(\%)\end{array}$ \\
\hline Po Valley fog & & & & -37.73 & 36.08 \\
Tenerife cloud & 72.8 & 0.0187 & 628.14 & -25.33 & 25.99 \\
Mace Head aerosol & 72.8 & 0.0200 & 99.01 & -40.10 & 71.45 \\
Biomass burning aerosol & 72 & 0.1700 & 11.86 & -30.49 & 36.08 \\
ACE Asia cloud & 72 & 0.0400 & 39.21 & -59.69 & 116.01 \\
SDS & 72 & 0.0540 & 293.08 & -66.44 & 193.95 \\
\hline
\end{tabular}

observed in the different environments reflect the differences in WSOC chemical composition. The curve for SDS is also shown for comparison illustrating that extracts from atmospheric aerosol are in general less surface active than SDS and surrogates for representing atmospheric aerosol should be chosen with caution.

This approach therefore may be used in appropriate models to determine the effect on cloud activation and droplet number of surface tension suppression by water soluble organic components in ambient aerosol, based on the dissolved carbon concentration. An alternative treatment is necessary if it is found that slightly-soluble, highly surface active compounds or insoluble, film-forming compounds are important in the atmosphere.

It should be noted that Mikhailov et al. (2004) demonstrated that proteins can form envelopes around salt particles, can affect the microstructure and porosity of mixed organic-inorganic particles, and influence the deliquescence, efflorescence, and hygroscopic growth of sodium chloride, ammonium nitrate, and ammonium sulfate particles even at low concentration levels. The study demonstrates that not only organics of limited/low solubility but also highly watersoluble biopolymers such as proteins and related (macro) molecules can significantly change the surface properties and water interactions of aerosol particles both by thermodynamic and kinetic effects. The thermodynamic effects may be efficiently included in Köhler model calculations. Studies should be carried out to directly establish the effects on $\mathrm{CCN}$ properties of such compounds. In addition, it remains to be investigated whether such nitrogen- containing compounds are present in sufficient numbers of fine particles or giant CCN (with reference to the study of Medina and Nenes (2004)) to significantly affect cloud activation and development.
Summary of the effects of component solubility and surface active properties

\section{Comments on solubility effects alone}

Because kinetic effects play an important role in cloud formation (Mordy, 1959; Nenes et al., 2001b), cloud model studies often shed more light on the activation properties of different types of particles than Köhler theory considerations. Shantz et al. (2003) used a cloud model to compare the activation of ammonim sulphate and adipic acid (a slightly soluble substance). They found that at given conditions, the maximum supersaturation inside the cloud was significantly lower and the activated number of particles significantly larger for ammonium sulphate. Ervens et al. (2005) and Abdul-Razzak and Ghan (2005) used cloud models to study the activation behaviour of mixed ammonium sulphateadipic acid particles. They found that adding small amounts $(\sim 5 \%)$ of ammonium sulphate adipic acid particles is enough to make the mixed particles to activate as efficiently as pure ammonium sulfate. Thus it is questionable whether the limited solubility plays a major role in atmospheric $\mathrm{CCN}$ activation. On the other hand, the experimental evidence presented by Raymond and Pandis (2002) indicates that wettable and non-wettable slightly soluble compounds behave qualitatively in a different manner in a $\mathrm{CCN}$ counter. Thus, it may turn out that the contact angle is a useful determinant of CCN behaviour: whether or not this is the case could be clarified with CCN chamber studies using mixtures of soluble salts and non-wettable slightly soluble organics such as those conducted by Raymond and Pandis (2003). These authors report that a little salt enhances the efficiency of activation for nonactivating particles. Taken together with the likelihood that multicomponent aerosols will tend towards the liquid phase (Sect. 4.1.3), this evidence suggests that the effect of limited component solubility on droplet number is unlikely to be significant. 


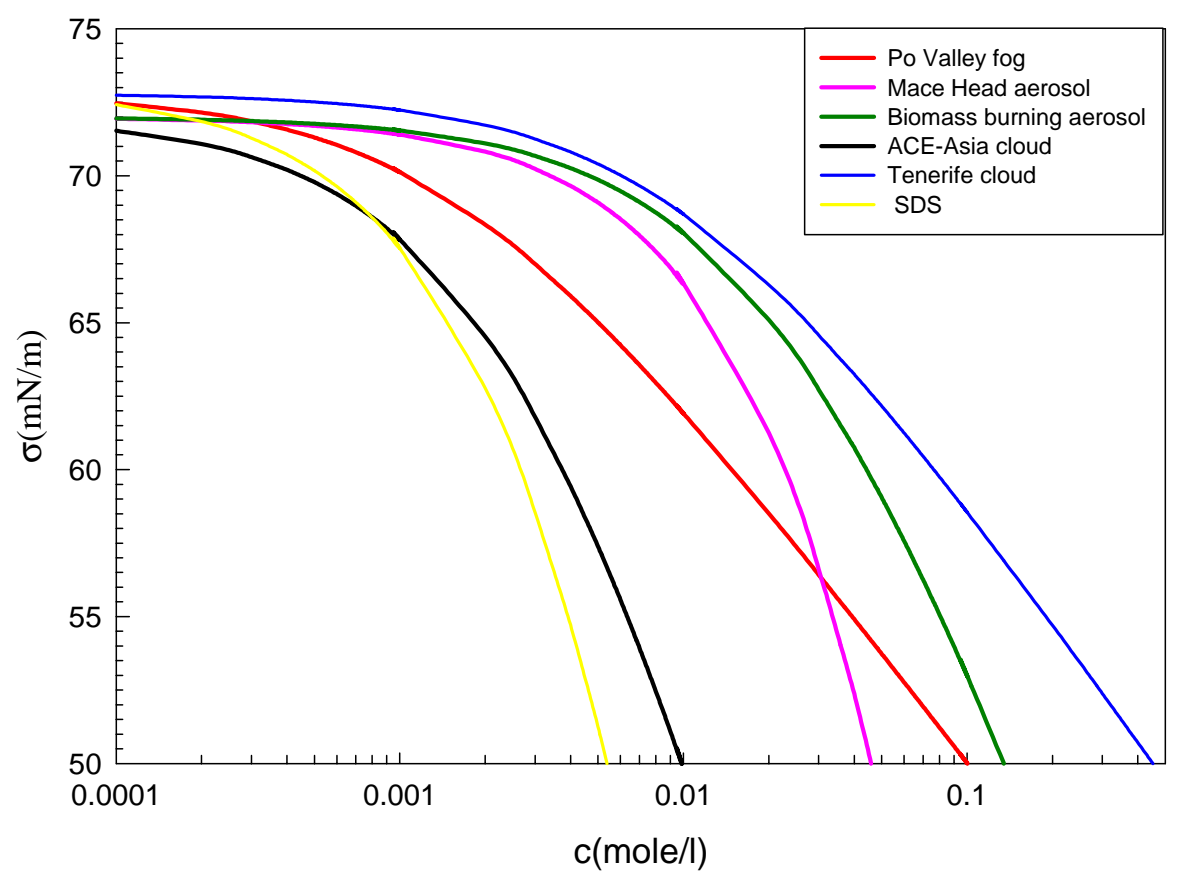

Fig. 12. Surface tension as a function of dissolved organic carbon concentration $(c)$ for fog, cloud and aerosol water extract samples from different environments. The curves were calculated with Eq. (14) (Syszkowski-Langmuir equation) and the data shown in Table 5 from data on real samples (cloud or fog droplets and aerosol water extracts.

\section{Effects on uptake kinetics}

The mass accommodation coefficient of water is used in cloud parcel models to describe the fraction of gaseous water molecules impinging on a growing cloud droplet that enters the liquid phase. However, despite decades of work on the subject, there is still significant disagreement in the aerosol and cloud physics communities on its magnitude. Laaksonen et al. (2005) contend that only the use of values similar to unity in the conventional formulation of droplet growth equations is consistent with laboratory findings. Whether the accommodation coefficient in such expressions corresponds to the actual mass accommodation coefficient of water or whether it is used to conveniently account for all combined kinetic effects is uncertain. According to IUPAC, the mass accommodation coefficient $\alpha$ is a measure of the ratio of the rate at which the molecules cross the interface region between the condensed phase and the gas phase normalised to the gas collision rate at the interface between the phases. The accommodation of molecules onto a growing droplet is the result of the dissipation of the available energy of the molecule moving on the surface such that, in the case of water, hydrogen bonds with the liquid molecules may form. The bond energy is of the order of $20 \mathrm{kJmol}^{-1}$ and this amount of energy can be dissipated thermally. The rate of this dissipation of energy and bond formation will compete with the rate of molecule efflux from the surface. Laboratory measurements of $\alpha$ tend to separate into values on the order of $10^{-2}$ (e.g. Chodes et al., 1974; Shaw and Lamb, 1999) and values close to unity (Davidovits et al. 2004; Winkler et al. 2004). For pure water and water containing polar species, $\alpha$ may be closer to unity (Mozurkewich, 1986; Laaksonen et al., 2005) but questions remain regarding the use of such values for growing haze droplets which might contain amphiphilic compounds, the evidence for which seems to be mounting (Tervahattu et al., 2005). Whether the presence of organic components, either as films or otherwise, actually suppresses the "accommodation coefficient", or some other aspect of growth kinetics is unclear; shielding of the water droplet surface by a condensed film might be expected to affect the kinetics of uptake of water molecules, as may condensed phase diffusional effects to particularly viscous substrates (although this is more likely to be important under sub-saturated conditions).

The effects of kinetic limitation on droplet activation are significant, all else being equal (e.g. Xue and Feingold, 2004): small values of $\alpha$ result in larger activated fractions since drops grow more slowly, allowing the supersaturation to build up higher. Feingold and Chuang (2002) showed significant reductions in activation and an associated droplet distribution broadening resulting from an assumed inhibition of the growth of some droplets covered by amphiphilic monolayers, and CCN closure studies have shown results which may be consistent with suppressed accommodation (see Sect. 5.2). The findings of Chuang (2003) in Mexico City are consistent with an effect on the kinetics of uptake, 
either truly through an accommodation effect or though some other suppression of equilibration. However, that fewer than $2 \%$ of the particles in this study belonged to this category may place a limit on the influence of such an effect. Medina and Nenes (2004) reported calculated suppression of drizzle formation resulting from even slight inhibition of giant $\mathrm{CCN}$ growth by film-forming compounds. It is possible therefore that low concentrations of film-forming compounds may significantly affect cloud processes by suppression of the activation and growth of specific subsets of the aerosol population.

Further laboratory evidence for disequilibrium behaviour has been provided by the electrodynamic balance study of Peng et al. (2001). The authors noted that glutaric acid particles absorbed water for several hours during deliquescence at 83-85\%; much longer than the time taken for other carboxylic acids to reach their equilibrium size.

Johnson et al. (2005) have recently presented results from a volatility-HTDMA (V-HTDMA) study, indicating a dramatic increase in the absolute water uptake of ambient accumulation mode particles (not merely an increase in the hygroscopicity of the residual) after previous desorption of volatile material at $60^{\circ} \mathrm{C}$. This indicates that there is a real suppression of water uptake by a volatile component on the timescale of the humidification in the instrument. Other than the highly water soluble ammonium nitrate, of commonly occurring aerosol constituents, $60^{\circ} \mathrm{C}$ is only adequate to volatilise organic components. It is therefore likely that a suppression of the water uptake by organic components is responsible for this effect.

Chan and Chan (2005) have reviewed mass transfer effects in hygroscopicity studies and recommend that future HTDMA studies report the residence time of particles in the humidification region to indicate whether kinetic limitations to mass transfer may be likely. This raises a further important issue. If HTDMA or CCN spectrometer measurements fail to allow sufficient time for particle equilibration, models based on prediction of equilibrium behaviour from composition will not be able to reconcile simultaneous composition and hygroscopicity or $\mathrm{CCN}$ activation spectrum measurements. Composition - hygroscopicity or composition - CCN closure will therefore be compromised. Ideally one would design an instrument to probe the response of a particle to saturation ratio in the same way that the particle will be exposed to a changing water vapour field below and in cloud. But such measurements, even when feasible, are very difficult to interpret given the range of possible aerosol particle histories in the atmosphere. Instead, one usually relies on models to predict particle growth and droplet activation. Accurate measurement of $\mathrm{CCN}$ (and CCN closure) under equilibrium conditions is not a sufficient condition for successful prediction of cloud droplet concentration. One still has to model the non-equilibrium conditions experienced by a population of particles growing and activating in a rising air parcel. Non-equilibrium size may derive from kinetic limitations associated with large particles, as well as from compounds that limit water vapour uptake. These effects must be captured accurately in cloud models, and possibly also in models that predict sub-saturated hygroscopic behaviour.

\section{Summary of surface activity effects}

As outlined in Sects. 3.1.4.1 and 3.1.4.2, organic components are almost ubiquitous in ambient aerosol particles and may affect cloud activation in a number of ways. A reduction in surface tension alone will lead to a reduction in critical supersaturation and hence to an increase in the number of activated droplets, all else being equal (Sect. 4.1.9). However, if partial (Sect. 4.1.7) or complete (Sects. 4.1.5 and 4.1.8) surface partitioning occurs in the atmosphere, the opposite effect will occur by reducing the number of molecules in solution and reducing the Raoult effect. It is evident that an inappropriate description of the surface active organic compounds in aerosol can lead to either overestimation or underestimation of the $\mathrm{CCN}$ number concentration and both enhancement and suppression of droplet number may be predicted. The fact that surface tension changes are the result of the aerosol chemical composition (a mixture of organic species having different solubility and different concentration which interact also with inorganic species), makes the choice of surrogates problematic, and both laboratory and modelling studies can for this reason obtain results which are far from the real properties of $\mathrm{CCN}$ in the atmosphere. It is likely that the effect on particle activation through both the Raoult and Kelvin terms will vary continuously in going from fairly soluble organic surfactants to insoluble film-forming surfactants. It is therefore necessary to establish the exact nature of the compounds which contribute to surface tension reduction in atmospheric aerosol and the component mass loading as a function of number. An alternative theoretical treatment of surface tension and its applicability is briefly addressed in the context of the primitive Köhler equation in Sect. 4.4.

4.2 Sensitivity of activation and droplet number predictions to variation in terms of the conventional Köhler equation

With increasing numbers of studies addressing the role of water soluble organics on activation, an assessment of the relative importance of the various composition-related terms in the Köhler equation on activation provides useful insight. An exercise of this kind can be considered with specific compounds in mind, or to explore parameter space where composition effects might be more, or less important. In a recent study, Ervens et al. (2005) performed multiple numerical simulations of activation in a rising updraught and considered the effects of surface tension, molecular weight, solubility and van't Hoff factor on the relative change in 
droplet number concentration compared to that obtained for a reference ammonium sulphate compound.

To briefly summarise, it was found that, in general, composition-related effects on drop number are more pronounced under conditions of low supersaturation, i.e. low updraught and high aerosol concentration. Under such conditions, the ability of a droplet to grow depends strongly on the solute and Kelvin terms (Eq. 2). The authors first considered changes due to each of the four parameters independently. As anticipated, low (fixed) values of surface tension resulted in strong increases in drop concentration. However, more realistically, droplet dilution during growth resulted in a steadily increasing surface tension, approaching that of pure water, and a significantly reduced effect. Molecular weight $M_{s}$ was shown to be a potentially important parameter because of its broad range of variability (more than an order of magnitude). Variations in van't Hoff factor are likely easier to quantify because of their smaller range of variability, and the fact that for many water soluble organic compounds, the van't Hoff factor is $\sim 1$. (For pKa values of about $4, M_{S}$ of $\sim 150 \mathrm{~g} / \mathrm{mole}$ and a maximum dissociation of 2 , the authors showed that only particles $>\sim 0.5 \mu \mathrm{m}$ exhibit dissociation $>10 \%$.) Solubility was shown to reduce drop concentrations significantly for values less than $\sim 25 \mathrm{gL}^{-1}$, and, unlike the other parameters, had a consistent effect for a wide range of updraughts and aerosol concentrations (also shown by Shantz et al., 2003). However, at higher solubilities, there was little effect on drop concentration.

When considering the more realistic, simultaneous effects of the various parameters, the authors noted that the negative relationship between surface tension and $M_{S}$ (Facchini et al., 1999a) creates opposing trends so that increases in drop concentration due to lower surface tension (associated with higher molecular weight species) are countered by the decrease in drop concentration associated with higher $M_{s}$. Investigation of the simultaneous effects of solubility, surface tension and $M_{S}$ suggested that for solubility greater than about $25 \mathrm{gL}^{-1}$, large reductions in drop number (relative to ammonium sulphate) are only expected at low updrafts (order $10 \mathrm{~cm} \mathrm{~s}^{-1}$ ), high aerosol concentrations (order $5000 \mathrm{~cm}^{-3}$ ) and $M_{s}$ greater than $\sim 400 \mathrm{~g} / \mathrm{mol}$. Under many conditions, composition effects appear to be closer to $\sim 15 \%$.

The authors attempted to reconcile their results with those of other work, including laboratory and modelling studies. Some of the high $(\sim 100 \%)$ quoted differences in drop number due to composition effects appear to be associated with calculations that assume equilibrium droplet growth (Phinney et al., 2003) rather than a coupled model that simultaneously calculates the dynamical supersaturation field resulting from expansion and cooling, and condensation growth. Under equilibrium assumptions, large particles are allowed to grow much more than they would in typical updraughts, and their uptake of water vapour reduces supersaturation and drop concentration commensurately. The effects tend to be strongest for the small particles where composition effects are most important. Other stronger excursions occur when high van't Hoff factors ( $i=3$ or 5) are assumed, or when the model or laboratory aerosol consists of $>90 \%$ of a relatively insoluble material (e.g. adipic acid).

\subsection{Co-condensation of soluble gases}

The most water soluble atmospheric trace gases can already condense into the aqueous solution droplets before the maximum supersaturation in a cloud has been reached and can therefore affect the activation behaviour and subsequent properties of the cloud droplets. Although the amounts of trace gases seem marginal, it is easy to see that they can provide a considerable amount of hygroscopic material to the droplets: at STP, 1 ppb of a trace gas corresponds to about $2.7 \times 10^{10}$ molecules per cubic centimetre of air, a similar number of molecules to that incorporated in 6400 ammonium sulfate particles having a diameter of $100 \mathrm{~nm}$. The most important trace gases in the atmosphere capable of influencing cloud properties are nitric and hydrochloric acids, and ammonia. Kulmala et al. (1993) made cloud model simulations of the effect of nitric acid on cloud drop activation. They showed that at mixing ratios of around $1 \mathrm{ppb}, \mathrm{HNO}_{3}$ can, depending on the aerosol size distribution and environmental variables, have a considerable effect on the activated fraction. They also calculated the effect of different amounts of nitric acid on Köhler curves in an open system (i.e. at constant $\mathrm{HNO}_{3}$ mixing ratios). The result is a constant depression of the Köhler curve by the amount of $v_{a}\left(P_{a} K_{a}\right)^{1 / 2}$, where $v_{a}, P_{a}$ and $K_{a}$ denote the acid's dissociation constant, partial pressure, and Henry's law constant, respectively.

A more realistic case than the open system is the closed system, in which the trace gas is depleted from the gas phase as it condenses. The Köhler equation then assumes the following form (Laaksonen et al., 1998):

$S=1+\frac{A}{r}-\frac{B}{r^{3}}-\frac{2 n_{a}}{b_{a} r^{3}\left(1+\sqrt{1+4 n_{a} /\left(C \beta r^{6}\right)}\right)}$

where $n_{a}$ is the total number concentration of the trace gas molecules in the system, $C$ is the aerosol particle concentration (the particle size distribution is assumed to be monodisperse), $\beta=K_{a} R T\left(4 \pi \rho_{w} / 3 M_{w}\right)^{2}$ and $b_{a}=4 \pi \rho_{w} / 3 M_{a} \nu_{a}$.

Depending on the particle size and concentration, and on the concentration of the trace gas, the Köhler curves can be radically altered with a flattened maximum shifted to larger sizes, and a possible local maximum and a local minimum at droplet sizes corresponding to the critical size calculated without the trace gas. Kulmala et al. (1997) suggested that at highly polluted conditions (5-10 ppb of nitric acid), the depression of the Köhler maximum, together with the possible effect from slightly soluble compounds, could lead to fogs or clouds containing micron sized, unactivated droplets.

The assumption of a monodisperse particle population is of course unrealistic at atmospheric conditions. Kokkola et al. (2003) extended the theory to polydisperse particle 
size distributions and found that as the $R H$ is sufficiently increased, an Ostwald ripening phenomenon occurs: the droplet population minimizes its surface free energy in such a way that the largest droplet of the population grows at the expense of the other droplets and ultimately, all of the trace gas is partitioned to the largest droplet. However, this is an equilibrium effect and needs an extremely slow cooling of the air (or increase of $R H$ ) to be realized. As the cooling rate is increased, the splitting of the size distribution moves toward smaller droplet sizes. Xue and Feingold (2004) also observed some degree of Ostwald ripening in their modelling study of the effect of nitric acid on droplet growth and showed that the associated broadening of the drop size spectrum could also have implications for the radiative properties of clouds.

Overall, cloud model studies have shown that the effects of nitric acid and other soluble trace gases on droplet activation depend heavily on the kinetics of cloud formation and cannot be explained solely with the Köhler theory. The $\mathrm{HNO}_{3}$ effect is more important at lower updraft velocities (Nenes et al., 2002) since the activated fraction decreases with decreasing updraught, so the trace gas causes a relatively larger enhancement in the activated fraction. Additionally, at low updraghts, the amount of $\mathrm{HNO}_{3}$ consumed per droplet is larger and the activation time of the cloud is longer, permitting more time for trace gas diffusion to the droplets.

Another important feature of the nitric acid effect is that it becomes much stronger at low temperatures (Laaksonen et al., 1997). This is due to the depression of the $\mathrm{HNO}_{3}$ vapour pressure as a function of decreasing temperature, allowing its uptake to the solution droplets already at relative humidities below $100 \%$. Also, the absolute amount of water vapour in the air is low at cold conditions, slowing down water vapour diffusion and increasing cloud activation times. Thus, at lower temperatures, nitric acid condensation starts earlier and occurs for a longer period before activation takes place, allowing for a larger fraction of the total nitric acid to be partitioned to the droplets.

As shown by Kulmala et al. (1998), the effects of nitric and hydrochloric acids on enhancement of droplet activation can be strongly boosted by co-condensation of ammonia. Furthermore, Hegg (2000) showed that the influence of ammonia becomes stronger with increasing aerosol acidity. However, judgement of the true role of ammonia in the activation process has proved to be difficult because in cloud model calculations, the assumption made concerning initial equilibrium or nonequilibrium of the trace gases strongly affects the results (Kokkola et al., 2003; Romakkaniemi et al., 2005). When nitric acid and ammonia exist in the air simultaneously, they can partition to the aqueous phase already at $R H$ of $90-95 \%$, depending on temperature. It is customary to equilibrate the aerosol e.g. at $95 \% R H$ with water vapour prior to a cloud model run. If the aerosol is also equilibrated with $\mathrm{HNO}_{3}$ and $\mathrm{NH}_{3}$, a large part of the gases may be partitioned to the largest droplets which will in any case be activated, and the effect of the gases on the activation can remain small. On the other hand, if it is assumed that the acid and the ammonia are completely in the gas phase at the start of the simulation, they will be transferred kinetically to the droplets during the cloud model run, and will condense more effectively to smaller droplets having a larger surface-to-volume ratio. In such a case the gases may have a very large effect on the activation. In the atmosphere, it depends on the history of the airmass which assumption is closer to the truth, and therefore, assessment of the impact of ammonia on cloud activation may be more difficult than assessment of the impact of nitric acid alone.

Taken together, environmental factors have a large impact on the effect of condensable trace gases on cloud drop activation and cloud properties. Nitric acid can have a large impact on activation especially in weak updrafts and at low temperatures. One factor limiting the effect of nitric acid is that the effect on cloud activation is largest when the gas phase nitric acid concentration is large and the aerosol concentration is small, yielding the most nitric acid per growing droplet. But the areas downwind of urban areas with high nitric acid concentrations often have large particle concentrations as well. Middle clouds such as altocumulus and altostratus may be especially susceptible to the effect of $\mathrm{HNO}_{3}$. However, for a given cloud liquid water content, whether the cloud albedo is affected also depends on the CCN concentration: in polluted regions, cloud albedos may already be so high due to the first aerosol indirect effect that further brightening caused by the trace gases is minimal. Regional and global assessments of the importance of the nitric acid effect have become possible with a recent parameterisation (Romakkaniemi et al., 2005); however, the role of ammonia needs to be clarified.

\subsection{Use of primitive form of the Köhler equation}

It is possible to utilise the form of the Köhler equation presented in Eq. (1) if the water activity can be directly evaluated. There are two classes of treatment which can allow this, evaluation of water activity based on laboratory measurements of derived properties and coupled thermodynamic treatments which predict the water activity based on fundamental properties of the components. In addition to being useful in their own right, predictions of activation behaviour using these techniques can be used to test the assumptions made in deriving the more simple forms of the Köhler equation.

\subsubsection{Determination of $a_{w}$ by osmolality measurement of atmospheric aerosol components}

This first form of prediction is heavily constrained by laboratory measurements and has several advantages. If the form of the Köhler Eq. (1) is used, the required information about the composition is limited to the water activity, partial molecular volume of water and surface tension of the growing droplet. If these properties are accessible to experimental 
determination as a function of droplet size, then the equilibrium behaviour of the growing droplet can be predicted. Kiss and Hansson (2004) suggested a simple approach to give reliable estimates of the Raoult term of the Köhler equation when calculating critical supersaturation $\left(S_{c}\right)$ for real atmospheric samples. Using laboratory measurement of the osmolality of simple electrolytes and sucrose, it was demonstrated that activation curves in accord with Köhler theory could be constructed. This technique is therefore possible for real atmospheric mixtures. Small errors are introduced into the Kelvin term by assuming the partial molar volume of water is equal to the molar mass of water divided by water density and, for non-surface active components, the surface tension may be assumed equal to that of water. For surface active components, this will be less true. If the laboratory determination of surface tension of multicomponent aerosol extracts may be reliably measured or estimated as a function of size (accounting for any surface excess due to the surface activity) this technique shows great promise.

\subsubsection{Prediction of multicomponent activation behaviour using a coupled model}

Models have recently been developed to predict the equilibrium composition of organic and inorganic multicomponent particles accounting for the effects of curvature on composition (Ming and Russell, 2002, 2004; Topping et al., 2005a,b). These models minimise the Gibbs free energy of the system including all solid and liquid components by expressing the Gibbs free energy in terms of the component chemical potentials, which in turn are expressed in terms of their activities.

$G=\sum_{i} n_{i} \mu_{i}, \mu_{i}=\mu_{i}^{0}+R T \ln \left(\gamma_{i}\right)$

$\mu_{i}(T, p)=\mu^{*}(T, p)$

where $G$ is the total Gibbs energy of the system, $n$ is the number of moles of component $i, \mu$ is the chemical potential and $\gamma$ is the activity. The activities are evaluated using a number of possible models. In the model of Topping et al. (2005a,b), the inorganic component activities are calculated using the mole fraction based Pitzer-Simonsen-Clegg ionic interaction model (Clegg et al., 1998) and the organic activities using the UNIFAC group contribution technique (Fredenslund and Prausnitz, 1975) with a variety of modified group interaction parameters. The energies of formation of all solids and an adjusted energy of formation of water is used to calculate the equilibrium phase state. A variety of minimisation algorithms can be applied which may require additional constraint. For example, the constrained minimisation algorithm of Topping et al. (2005a,b) uses gradient information from the Gibbs-Duhem relation for convergence:

$\frac{\delta G}{\delta n_{i}}=\mu_{i}$
To account for curvature, it is necessary to evaluate the multicomponent density using appropriate mixing rules to evaluate the partial molar volume of water in Eq. (1):

$v_{w}=\frac{M_{w}}{\rho_{\mathrm{sol}}}\left(1+\frac{x_{s}}{\rho_{\mathrm{sol}}} \frac{\mathrm{d} \rho_{\mathrm{sol}}}{\mathrm{d} x_{s}}\right)$

where $x_{s}$ is the solute mole fraction and $\rho_{\text {sol }}$ is the solution density. In addition, the multicomponent surface tension must be evaluated.

For many organic species neither the pure component nor binary aqueous surface tension data are available. It is therefore frequently necessary to predict these quantities from fundamental molecular information. A number of surface tension mixing rules exist to combine these predicted data. The reader is referred to Topping et al. (2005b) for a discussion of the available techniques. Such surface tension predictions, though internally consistent within the model framework (some requiring the same activity predictions), remain to be validated against real atmospheric data. Section 4.1 details many of the pitfalls which may be encountered if inappropriate components are chosen.

The use of such models has thus far only been reported for sub-saturated conditions, but they are capable of explicitly evaluating the equilibrium size and composition at any saturation ratio according to the primitive form of the Köhler Eq. (1). The numerical solver in the Aerosol Diameter Dependent Equilibrium Model (ADDEM) of Topping et al. (2005b) has been modified slightly to extend its range of operation to include saturation ratios greater than unity. In subsaturated application, the algorithm equates the $R H$ and water activity before iterating the composition within the Kelvin term loop. The initial solution would become unstable when attempting supersaturated solution. Therefore, the energy of formation of water is initially nudged and relaxed throughout the optimisation to keep the water activity below unity. Additional constraints are constructed to ensure solution either side of the critical radius.

Since the model explicitly considers non-ideal effects, it can predict the equilibrium composition across a wider range of humidity than most other particle equilibrium calculations. Figure 13a shows the Köhler curve prediction for simple one and two electrolyte particles accounting for all non-ideal interactions and multicomponent density and surface tension. In this case, using the surface tension mixing rule presented by Chen (1994), the model can be successfully used down to low $R H$, showing the increased deliquescence $R H$ with decreasing size, the stepped deliquescence of mixed salt particles and the increased water content of the mixed salt particle in both sub- and supersaturated regimes with respect to $\left(\mathrm{NH}_{4}\right)_{2} \mathrm{SO}_{4}$ particles. Panel (b) shows the predicted behaviour of a mixed $\left(\mathrm{NH}_{4}\right)_{2} \mathrm{SO}_{4} /$ glutaric acid particle accounting for non-ideality and, multicomponent density and surface tension. In this case, the surface tension is taken from the parameterisation of Gaman et al. (2004) based on 
experimental data, eliminating sensitivity to inaccuracies in surface tension prediction methods.

However, care will still need to be taken when comparing with laboratory $\mathrm{CCN}$ measurements since attaining equilibrium water content of glutaric acid has been shown to take several hours (Peng et al., 2001). Panel (b) also highlights an interesting distinction. If all particles at a given dry size contain both ammonium sulphate and glutaric acid, but at one end of the composition range the ratio was $1: 4$ and at the other end was $4: 1$, it is evident that the particles must be considered to be quasi-internally mixed. That is, although each component is present in all particles, the variable component mass fractions lead to significant variation in the derived property of interest (in this case CCN activity). Significant error in predicted behaviour would be introduced using either an internal or external mixture.

In principle, such models can implicitly account for the response of the particle to the gaseous saturation ratio of all semi-volatile organic and inorganic species including water. This requires the simultaneous solution of the Köhler equation for the saturation ratio of each species as conducted for $\mathrm{HNO}_{3}$ by Kokkola et al. (2003). In addition, the dissolution of slightly soluble compounds is explicitly resolved by the consideration of solid equilibration. These models, though numerically expensive, may therefore be useful developments to investigate the effects of aerosol composition on droplet activation.

\section{Reconciliation of composition, derived aerosol prop- erties and cloud droplet number}

\subsection{Aerosol composition - hygroscopicity closure}

The contribution of inorganic and organic aerosol compounds to the hygroscopic growth of ambient particles has been investigated in so-called hygroscopicity closure studies comparing HTDMA measurements of particle water uptake to predicted water uptake inferred from chemical composition measurements and corresponding theoretical growth factors of the ingredient mixture. Most studies model only the water uptake of the inorganic aerosol fraction, as the wateruptake of the organic aerosol fraction is generally not known, and attribute the difference between measured and predicted water uptake to the aerosol organic fraction. Studies by Saxena et al. (1995) and Berg et al. (1998a) in urban or nearurban locations show that inorganic salts may account for the entire measured water-uptake indicating that primary organic compounds do not contribute significantly to hygroscopic growth. In aged polluted environments on the other hand, organics compounds were found to significantly contribute to the total water uptake of aerosol particles (Saxena et al., 1995; Dick et al., 2000; Gysel et al., 2004; Weingartner et al., 2004), except for a study by Swietlicki et al. (1999), where a significant contribution was only found dur-

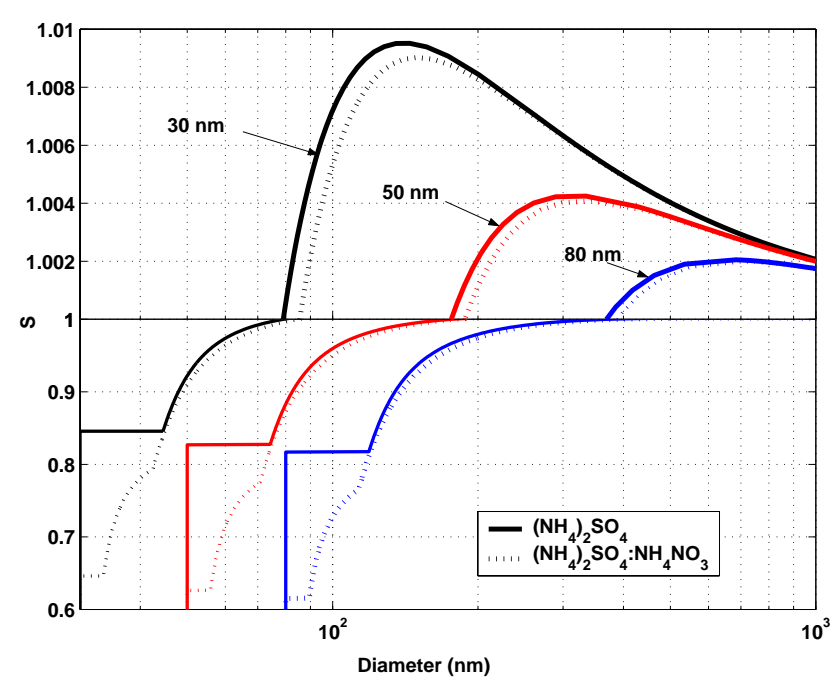

(a)

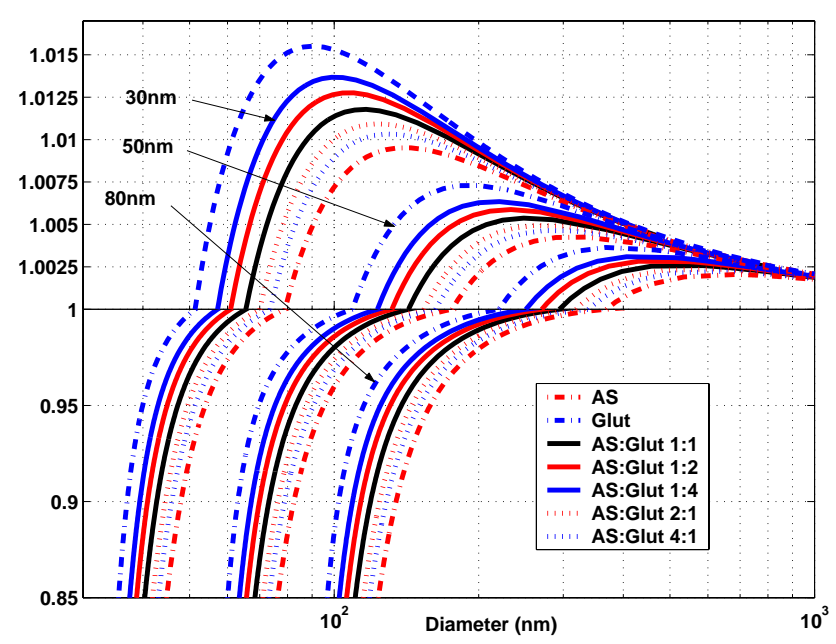

(b)

Fig. 13. (a) Equilibrium behaviour of ammonium sulphate and 1:1 mole ratio ammonium sulphate/ammonium nitrate particles of different initial dry sizes, (b) Activation curves of pure ammonium sulphate (AS) and glutaric acid (Glut) particles of different sizes and of various molar ratios of mixed particles.

ing a photochemically active period. McFiggans et al. (2005) used a combination of impactor and AMS measurements to describe the composition distributions in polluted continental air masses and used a model incorporating the primitive form of the Köhler equation to predict HTDMA measured $G F_{D}$. It was found that the variability in $G F_{D}$ was largely dominated by the inorganic:organic mass ratio at any given size and that the water associated with the organic fraction was modest and relatively invariant between functional representations of the organic component at two comparable locations. 
Table 6. Recent aerosol-to-CCN closure studies.

\begin{tabular}{lllll}
\hline Investigator & $\begin{array}{l}\text { Aerosol Chemical } \\
\text { Speciation }\end{array}$ & Campaign & $\begin{array}{l}\text { CCN activity } \\
\text { of organics }\end{array}$ & $\begin{array}{l}\text { Predicted-to- } \\
\text { observed Ratio }\end{array}$ \\
\hline Covert et al. (1998) & HTDMA and impactor & ACE-1 & $\begin{array}{l}\text { Examined } \\
\text { Examined }\end{array}$ & 1.2 \\
Dusek et al. (2003) & HTDMA and impactor & ACE-2 & Assumed inactive & 1 to 4 \\
Chuang et al. (2000) & Impactor & ACE-2 & Assumed inactive & 1.3 \\
Zhou et al. (2001) & HTDMA & AOE-96 & Assumed inactive & 1.0 \\
Cantrell et al. (2001) & Impactor & INDOEX & Examined & 1.3 \\
Snider et al. (2003) & HTDMA and Impactor & ACE-2 & Examined & 1.0 \\
Roberts et al. (2002) & HTDMA and impactor & CLAIRE-98 & Axsumed Active & 1.1 \\
VanReken et al. (2003) & AS assumed & CFACE & Assumed & 1.1 \\
Rissler et al. (2004) & HTDMA & CLAIRE-01 & Examined & \\
\hline
\end{tabular}

\subsection{Aerosol - CCN and aerosol - droplet closure studies}

As introduced in Sect. 3.2.2, CCN measurements are often used to evaluate consistency among measured and derived estimations of a single-supersaturation $\mathrm{CCN}$ concentration, among measured and derived estimations of a $\mathrm{CCN}$ activation spectrum, and among measured and derived values of the cloud droplet number concentration. The former two comparisons will be referred to as aerosol-to-CCN closures, and the latter as an aerosol-to-droplet closure.

Numerous field studies have been conducted with emphasis on evaluating aerosol-to-CCN consistency. With few exceptions these demonstrate that predicted $\mathrm{CCN}$ concentrations exceed those measured, but in all cases the uncertainties are comparable to the departures of the predicted-toobserved $\mathrm{CCN}$ ratios from unity, shown in the fifth column of Table 6. However, since even a $10 \%$ departure of the predicted-to-observed ratio from unity could manifest as a substantial error in model predictions of aerosol indirect effect, emphasis remains focused on improving both the precision and absolute accuracy of the measurements.

Further confounding aerosol-to-CCN closure is the unknown CCN activity of the organic aerosol fraction. For example, Dusek et al. (2003) analyzed data collected in southern Portugal and showed that the water-soluble organic carbon constitutes less than $10 \%$ of the total particulate mass. In recognition of this, Dusek et al. (2003) ignored contributions of the organic carbon to CCN activation. Similarly, Roberts et al. (2002) ignored the CCN activity of the organic fraction in their examination of Amazonia wet season aerosol where the total organic carbon mass fraction exceeds $50 \%$. An increase of the predicted-to-observed ratios to values larger than that presented in Table 6 was demonstrated by both Dusek et al. (2003) and Roberts et al. (2002) in sensitivity studies which considered surface tension reduction by hypothesized organic aerosol components (Facchini et al., 1999b). Enhancements of the predicted CCN activity could also occur if the two assumptions often made in the interpretation of HTDMA data are found to be incorrect: 1) solute dissolution is complete when particles are sized at elevated $R H$ in an HDTMA and 2) dried aerosols in an HDTMA are desiccated, compact and spherical.

Another confounding issue is the possible influence of film-forming (amphiphilic) compounds (see Sect. 4.1). Bigg (1986) reported unexplained delays in activation in a CCN chamber that could be attributed to film-forming compounds. During the Indian Ocean Experiment, Cantrell et al. (2001) showed poor aerosol-to-CCN closure on days characterized by high $(\sim 25 \%)$ organic fraction but were able to improve closure if a mass accommodation coefficient of $10^{-4}$ was assumed (c.f. the inferred values of $10^{-5}$ of Chuang, 2003). As noted in Sect. 4.1 factors other than the mass accommodation coefficient might also be responsible for poor aerosolto-CCN closure but to the extent that kinetic effects are important, they do shift the predicted-to-observed ratios closer to unity.

In contrast to the supersaturation imposed within a $\mathrm{CCN}$ chamber, supersaturation in an ascending cloud parcel is sensitive to the competition between vapour depletion, due to droplet growth, and the rate with which vapour is made available via adiabatic expansion (Sect. 3.1.1). As a consequence, closure studies examining the aerosol-to-cloud transition require an updraft velocity, in addition to a $\mathrm{CCN}$ activation spectrum. The aerosol-to-droplet closure studies also require droplet concentrations sampled from cloud regions unaltered by either entrainment mixing or scavenging by precipitation. Table 7 shows that three of four aerosol-to-droplet closure studies report reasonable consistency between prediction and observation, while the earliest of these reported substantial overprediction. A factor which complicated the interpretation of the ACE-1, ACE-2 and CFACE aerosol-to-droplet studies was the logistical problem of using one aircraft to make both sub-cloud aerosol and in-cloud droplet concentration measurements. Furthermore, both the airborne- and the ground-based studies (i.e. Hallberg et al., 1997) were fraught with the challenges of obtaining unbiased measurements of cloud droplet concentration (Burnet and Brenguier, 2002) and updraft velocity (Guibert et al., 2003). 
Table 7. Recent aerosol-to-drop number closure studies.

\begin{tabular}{|c|c|c|c|c|c|}
\hline Investigator & Campaign & $\begin{array}{l}\text { CCN activity } \\
\text { of organics }\end{array}$ & $\begin{array}{l}\text { No. of flights } \\
\text { or cases }\end{array}$ & $\begin{array}{l}\text { Mass } \\
\text { Accommodation } \\
\text { Coefficient }\end{array}$ & $\begin{array}{l}\text { Predicted-to- } \\
\text { observed Ratio }\end{array}$ \\
\hline Hallberg et al. (1997) & GCE-EUROTRAC & Examined & 2 & 0.04 & 2 to 20 \\
\hline Yum et al. (1998) & ACE-1 & $\begin{array}{l}\text { Not Applicable } \\
\text { (used measured CCN) }\end{array}$ & 16 & 1.00 & 1.2 \\
\hline Yum et al. (1998) & ACE-1 & $\begin{array}{l}\text { Not Applicable } \\
\text { (used measured CCN) }\end{array}$ & 16 & 0.04 & 1.6 \\
\hline Snider and Brenguier (2000) & ACE-2 & $\begin{array}{l}\text { Not Applicable } \\
\text { (used measured CCN) }\end{array}$ & 9 & 1.00 & 0.9 \\
\hline Snider et al. (2003) & ACE-2 & Examined & 5 & 0.10 & 1.2 \\
\hline Conant et al. (2004) & CFACE & Examined & 20 & 0.06 & 0.9 \\
\hline
\end{tabular}

Excluded from Table 7 are closure exercises employing parameterised, as opposed to parcel model, droplet activation codes. As was mentioned in Sect. 3.2.2 such model validation exercises build on a successful comparison of measured and parcel-model-predicted droplet concentration values; examples of the former include Meskhidze et al. (2005) and Peng et al. (2005).

Summarising, with few exceptions, the aerosol-to-CCN closure studies show that predicted $\mathrm{CCN}$ exceed direct $\mathrm{CCN}$ measurements. This pattern of overprediction is repeated in the aerosol-to-droplet closures, but here our limited understanding of the mass accommodation coefficient has more direct consequences because it affects the rate of supersaturation depletion in a cloud parcel. Secondary effects may also arise from model formulations of the particle sizedependence of the mass accommodation (Hallberg et al., 1997; Feingold and Chuang, 2002). For any one field experiment, the overprediction generally does not exceed error bounds associated with the predicted-to-observed ratios, but the predominance of ratios greater than unity is indicative of a bias in the measurements (aerosol, $\mathrm{CCN}$, or droplets) or in the application of the Köhler or cloud parcel theories. This apparent bias is amplified by several percent by the parameterisation of surface tension Facchini et al. (1999b) and by the relaxation of assumptions often made in the analysis of HTDMA data. Of course, if other effects of organic components were to cancel out or overwhelm the surface tension reduction, closure would be improved.

\section{The effect of aerosol properties on droplet formation in a range of locations}

6.1 Offline composition analyses from a variety of locations

This section presents size-segregated chemical composition of different types of aerosol collected in several locations worldwide, in a form which can be used for cloud mod- elling purposes. Sampling has been carried out by multistage cascade impactors with a common analytical protocol, aimed at mass closure and providing total mass, water soluble inorganic ions (WS_INORG), total carbon, elemental carbon, water-soluble organic carbon (WSOC). WSOC has been characterised by functional group analysis based on liquid chromatography and nuclear magnetic resonance $\left({ }^{1} \mathrm{HNMR}\right)$ and its composition has been reduced to a set of model compounds following the procedure described by Fuzzi et al. (2001). Insoluble mass fraction (INS) is calculated as the difference between total mass (obtained by weighting the filters) and total soluble mass. The details of sampling and analysis are reported in the papers referenced below. In general, in the sub-micron size range, INS mainly comprises insoluble organic species; while in the coarse size fractions, INS is mainly inorganic crustal components.

Figure 14 shows a high degree of variability between locations and size fractions in the chemical composition of organic and inorganic water soluble components, the insoluble fraction and total aerosol mass. Despite the differences, all aerosol types are characterised by a high content of organic matter in the sub-micron fraction (in this size range organic matter comprises the WSOC and almost the entire INS fraction). The higher organic content in the sub-micron fraction observed in the urban site (Bologna, Italy) in winter can be explained by enhanced combustion sources and, during the dry summer, the coarse fraction is richer in insoluble crustal components. The hygroscopic fraction in the accumulation mode is mainly dominated by inorganic salts while WSOC represents the major component in the size range below $0.14 \mu \mathrm{m}$. In the aerosol from the boreal forest, the fine mode, including particles smaller than $0.42 \mu \mathrm{m}$, is composed in almost equal parts of water-soluble organic and inorganic species. With increasing size, the contribution of water-soluble organic carbon decreases in favour of the inorganic species and water-insoluble components. At the pasture site, Brazil, the aerosol collected during the biomass burning (dry) season has a similar percent composition as the 


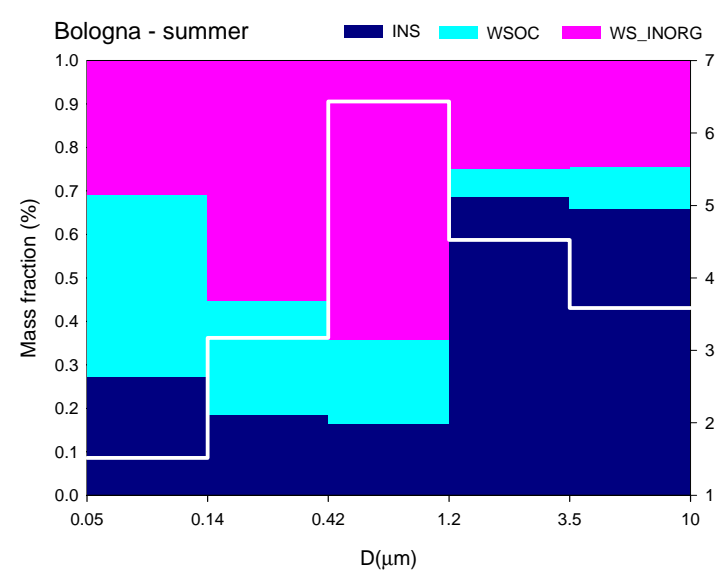

(a)

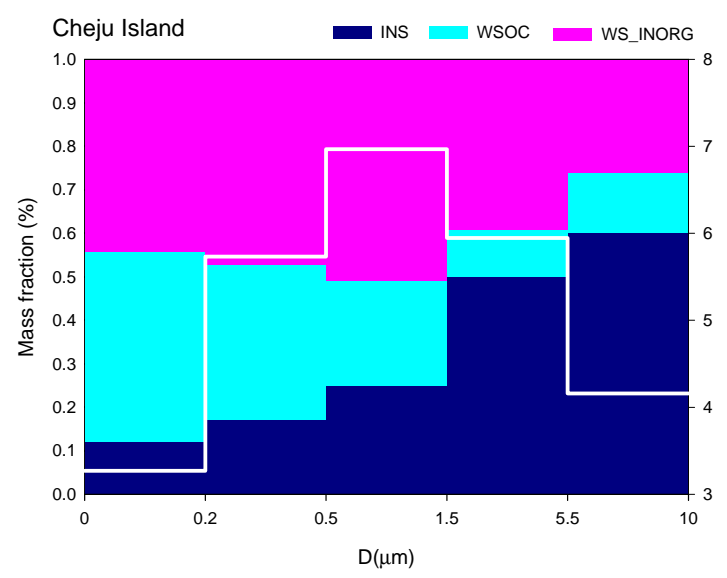

(c)

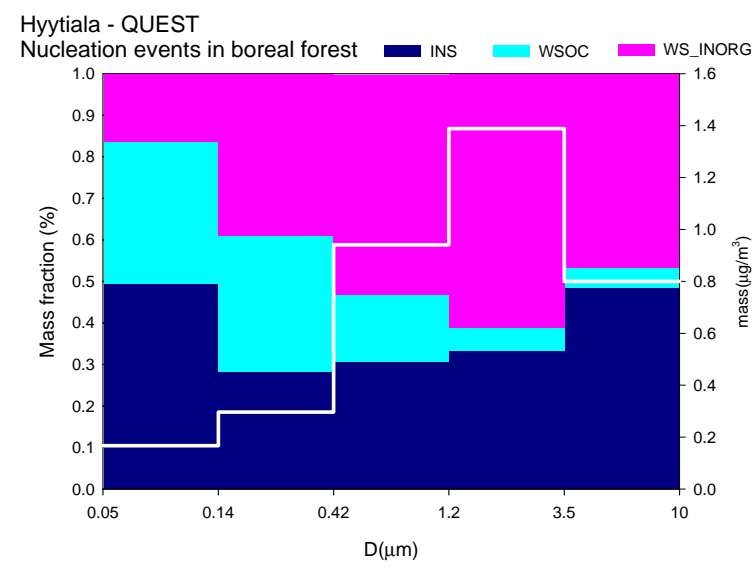

(e)

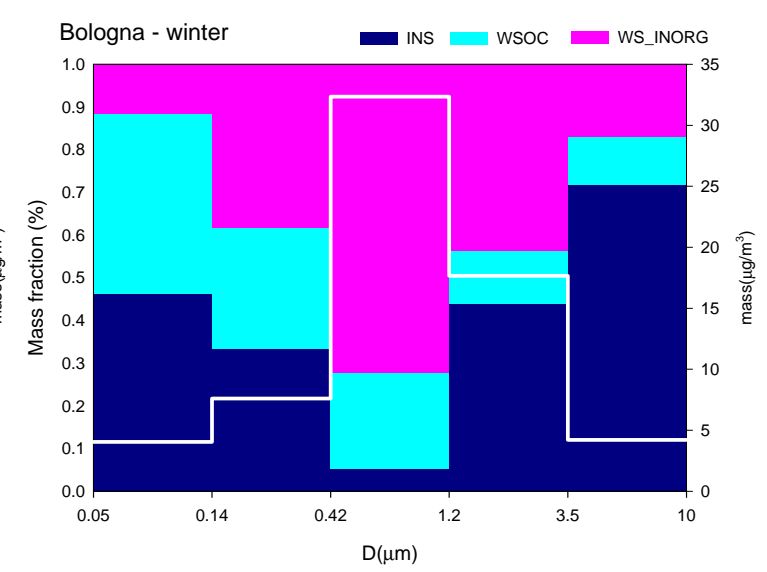

(b)

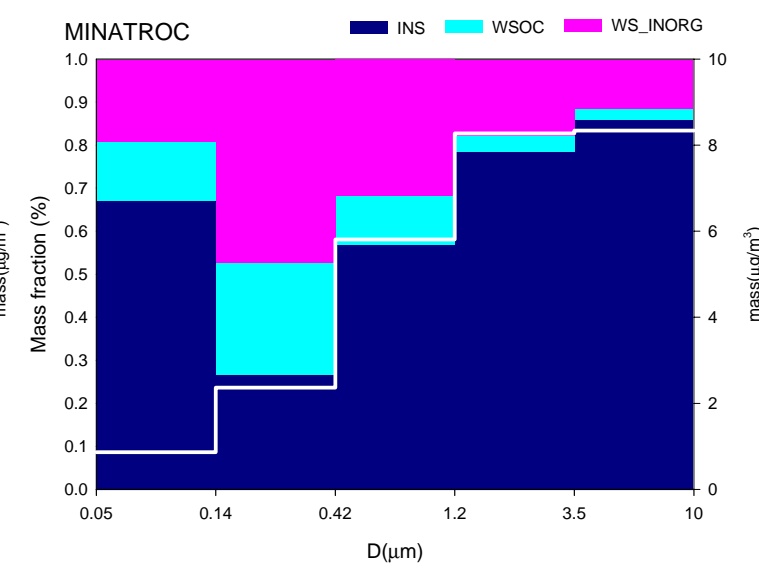

(d)

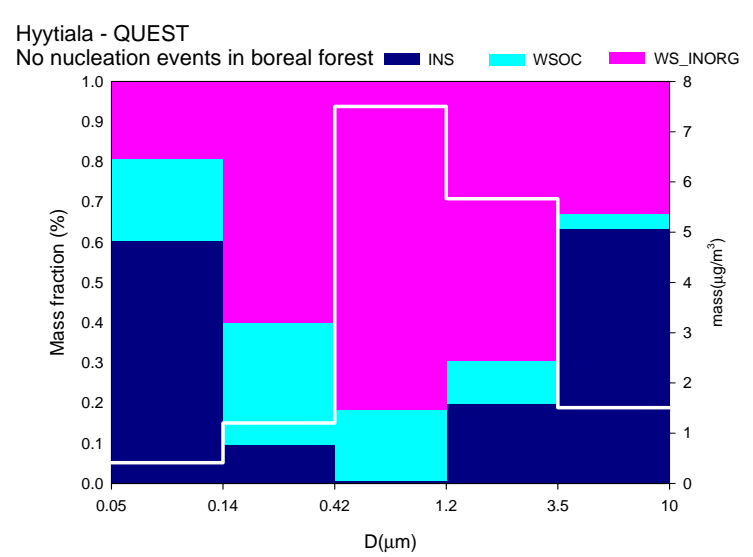

(f)

Fig. 14. Mass size distribution (right axis) and size-segregated chemical composition (left axis) of aerosol at different locations. (a) Bologna, summer, (b) Bologna, winter, (c) Jeju Island, Korea (d) Monte Cimone, dust event (e) Hyytiälä, nucleation event., (f) Hyytiälä, no nucleation event. 


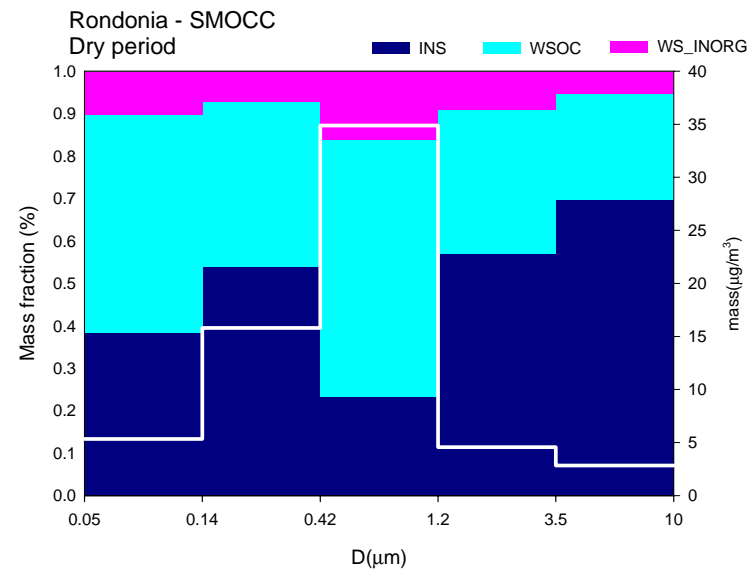

(g)

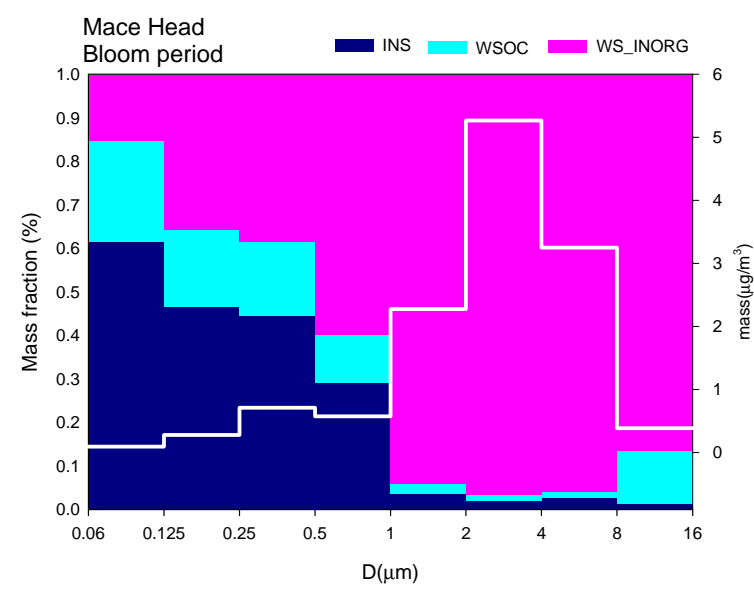

(i)

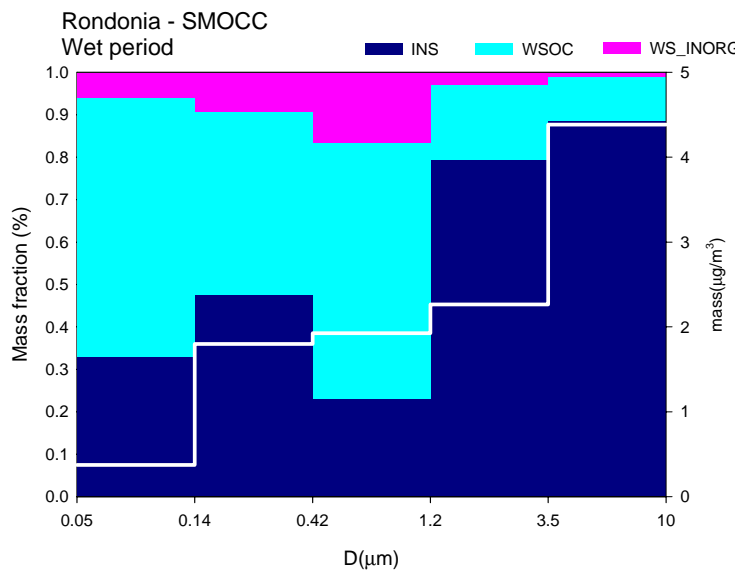

(h)

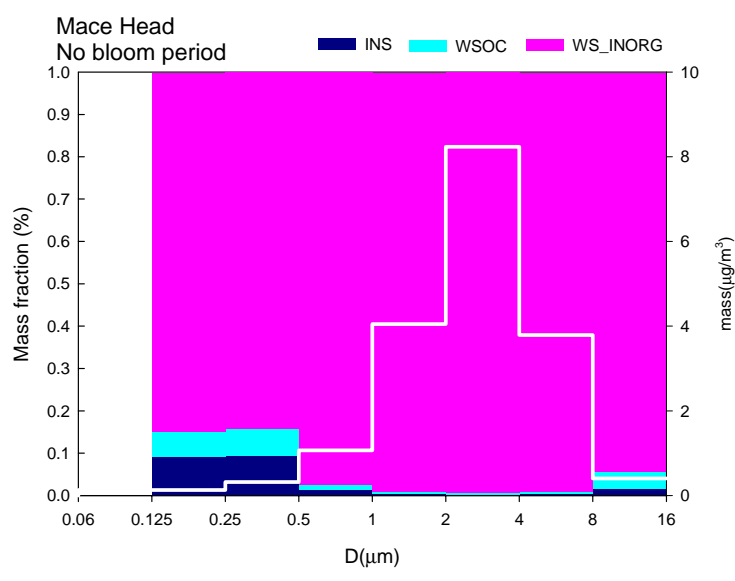

(j)

Fig. 14. (g) Rondonia, biomass burning season, (h) Rondonia, wet season, (i) Mace Head, Ireland summer; (j) Mace Head, Ireland winter. WSOC $=$ water-soluble organic matter, WSINORG = water-soluble inorganic matter and INS = insoluble fraction.

aerosol collected during the wet season, but the total aerosol load differs by one order of magnitude. WSOC is the major soluble component, also in the accumulation mode, while the soluble inorganic ions represent from a few percent up to $15 \%$ of the total aerosol mass. The sub-micron marine aerosol chemical composition is dominated by organic fraction (mainly insoluble) during summer while during the winter period the main chemical component of the sub-micron fraction is sea salt. As expected, the coarse fraction is dominated by soluble sea salt components in both seasons.

It can be seen that the smallest size fraction exhibits significant variability between the locations in the ratios between insoluble, water soluble inorganic and water soluble organic components. This is the fraction where the activation threshold dry size will most likely be found and hence the composition will impact greatly on droplet activation.

\subsection{Marine Boundary Layer (MBL)}

Since the oceans impart a low albedo to vast areas of the planet, influences of marine aerosol may account for a large component of the global radiative budget. The marine aerosol - cloud system has frequently been assumed to be one the simplest to describe. In the marine boundary layer, the number of particles large enough to act as cloud condensation nuclei is set by several factors, including entrainment from the free troposphere and emission of sea salt droplets from the ocean surface.

A number of studies have pointed to entrainment from the free troposphere as the major source (by number) of mixed sulfate-organic particles in the marine boundary layer (e.g. Bigg et al., 1984; Van Dingenen et al., 1999; Liu et al., 2001). Indeed, anthropogenic and other continental sources 


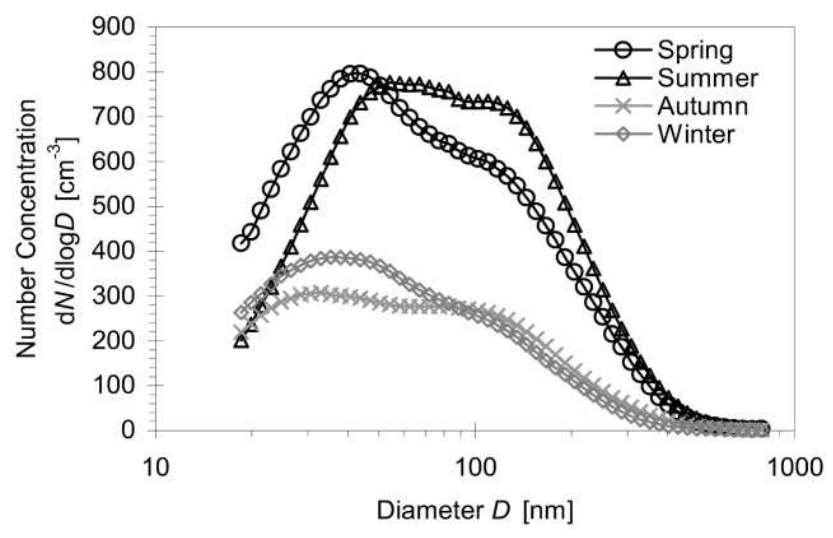

Fig. 15. Number size distributions for the high Alpine site JFJ (3580 $\mathrm{m}$ a.s.1.). Averages for the four seasons, spring 1998, summer 1997, autumn 1997, and winter 1997/1998 are shown. The spectra are well parameterised with the sum of two lognormal size distributions having modal diameters and standard deviations that are surprisingly constant throughout the year. The seasonality is due to variations in the number concentrations in the Aitken and Accumulation mode. Adapted from Weingartner et al. (1999).

of sulfate can be important far from land (Heintzenberg et al., 2000; Patris et al., 2000; Putaud et al., 2000). Downward mixing is important and frontal passages and other meteorology are very important in determining the number of sulfate particles available (Bates, 1999). Single particle data indicate that these entrained particles from the free troposphere contain organics internally mixed with sulfate (see Fig. 8 and De Bock et al., 2000).

Sea salt particles contribute a significant minority of the number of cloud condensation nuclei in the marine boundary layer. The contribution is strongly dependent on wind speed, with for example about 70 sea salt particles $\mathrm{cm}^{-3}$ for $17 \mathrm{~ms}^{-1}$ winds (O'Dowd et al., 1997; Mason, 2001). This is a change from the previously widely-held view that sea salt particles are present at only about $1 \mathrm{~cm}^{-3}$. Instead, measurements of the dependence of the number of particles on wind speed have shown that many of the submicron particles must be sea salt (O'Dowd et al., 1999). Single particle analysis using both electron microscopy and mass spectrometry at Cape Grim showed that most particles larger than $130 \mathrm{~nm}$ at that location were sea salt (Murphy et al., 1998). Ionic analysis of size selected samples has led to the conclusion that sea salt dominates the light scattering by submicron particles across wide areas of the ocean (Quinn et al., 2000). Volatility and electron microscope analysis have both shown sea salt particles down to well below $100 \mathrm{~nm}$ (e.g. Clarke et al., 2003). These sea salt particles also contain an estimated 5 to $50 \%$ organic matter (Middlebrook et al., 1998; Bigg and Leck, 2001; Putaud et al., 2000; O'Dowd et al., 2004; Lohmann and Feichter, 2005). At least some of the organic material is at the surface of the particles (Tervahattu et al., 2005). As is detailed above, the effects of organic components on both the mass of soluble material in, and the surface tension of, an activating aerosol particle may be significant. The surfactant properties of the organic layer on the ocean surface may also influence the number of wind-generated particles (MacIntyr, 1974; Slauenwhite and Johnson, 1999).

Despite these general conclusions, there are only a few quantitative measurements of the relative contributions of sea salt to cloud condensation nuclei. An assessment of the relationship of $\mathrm{CCN}$ to wind speed found that a $10 \%$ increase in wind speed would increase the number of $\mathrm{CCN}$ in the marine atmosphere by less than 5\% (Bigg et al., 1995). At Macquarie Island $\left(54^{\circ} \mathrm{S}\right)$, volatility analysis showed about half the CCN were sea salt (Kreidenweis et al., 1998; Murphy et al., 1998). This is a region of high wind speeds and very low anthropogenic influence, so the global contribution of sea salt will be less. Tandem humidity measurements have consistently found a much smaller fraction of sea salt. For example, particles with humidity-dependent growth characteristic of sea salt were found at about $150 \mu \mathrm{m}$ only 13,35 , and $16 \%$ of the time on cruises through the Pacific, Southern, and Arctic Oceans, respectively (Berg et al., 1998b; Zhou et al., 2001). Even when present, sea salt particles were usually a minority so the inferred overall abundance of sea salt particles was only a few percent by number. These humiditydependent data may represent a lower limit because the organic material present in the sea salt particles can reduce their hygroscopic growth to something nearer to sulfate particles (Randles et al., 2004).

Section 3.1.4 has demonstrated the complex dependence of activation behaviour on the interacting aerosol size, composition and updraught in the marine environment. The presence of organic components in seasalt particles will only increase the complexity of such dependencies. It is unclear whether the generally reasonable agreement in marine CCN and droplet closure studies (Sect. 5.2) using relatively simple interpretive treatment is indicative of relative insensitivity to compositional complexity as may be illustrated by the analysis in Sect. 3.1.3.

\subsection{Free Troposphere (FT)}

Cloud processes in the free troposphere often involve the ice phase, through cirrus clouds, or in mixed phase frontal or convective systems. Aerosol-cloud interactions in such cloud types are beyond the scope of this review. However, supercooled water clouds are often involved in the formation of such clouds before freezing takes place and are often key to understanding the processes governing the transportation of material from the boundary layer to the free troposphere. There are few studies on warm cloud activation in such systems, though measuring sites located on mountain tops are often suitable for their study.

Aerosol size distributions at locations such as the Jungfraujoch (Swiss Alps, $3580 \mathrm{~m}$ above sea level), show that the shape of the Aitken and accumulation aerosol size 


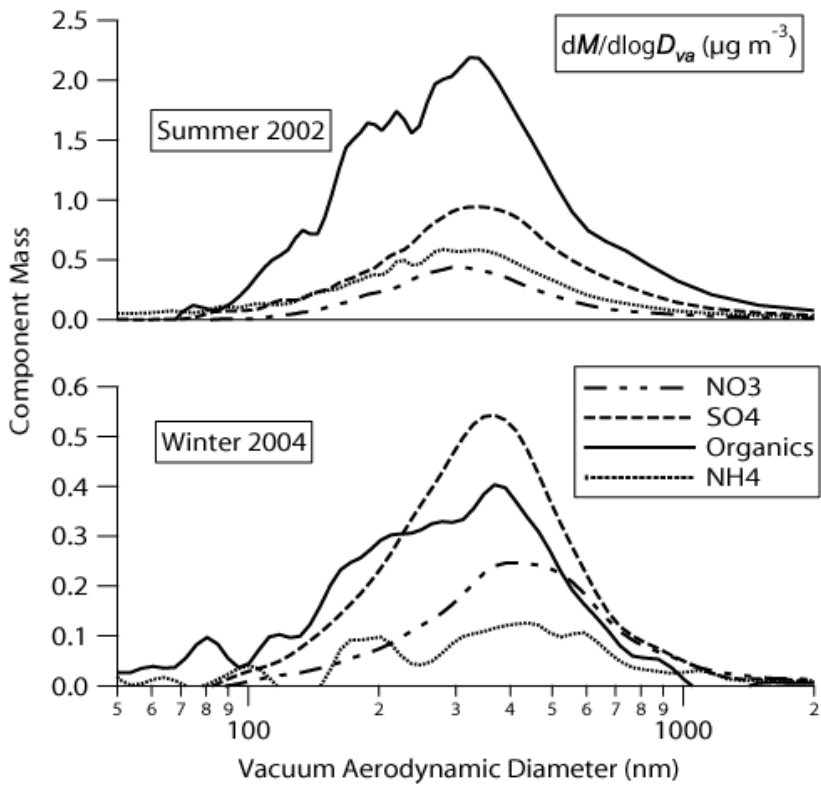

Fig. 16. Mass distributions for summer 2002 (top, McFiggans et al. (2005)) and winter 2004 (bottom, unpublished) as obtained with an Aerodyne AMS.

distributions are relatively invariant throughout the year (Fig. 15), though the absolute magnitude of the two modes vary independently by an order of magnitude (Weingartner et al., 1999). The seasonal variation in number concentration is due to enhanced thermal convection in the summer months, as analyzed in detail by Lugauer et al. (2000). Valley venting thus results in substantially higher number concentrations than during free troposphere conditions, while the normalized size distribution is virtually unchanged.

Compositional analysis using an AMS has shown the presence of an internally mixed mode of sulphate, nitrate and organic material, the relative fractions are variable and have a seasonal dependence such that the organic fraction is smaller in winter-time. As can be seen in Fig. 16 there is evidence of an increased organic mass fraction in particles below $150 \mathrm{~nm}$ in size, though the differences in the average component mass distributions arise from the intermittent presence of an enhanced Aitken mode comprised of organic material. These events will have a significant impact on the activation process as the composition change occurs in the size range where the activation cut off is to be expected (see below).

However, for much of the time, HTDMA measurements show that a single growth mode is dominant at any given dry particle size whose relative component mass controls the hygroscopic properties of the particles as shown in Fig. 17 (Weingartner et al., 2002). This growth factor is higher in winter than in summer, which can be explained by the greater fraction of organic material as shown by the AMS measurements (Fig. 16), with organic material having a lower growth factor than inorganic material. At any given time, the mea-

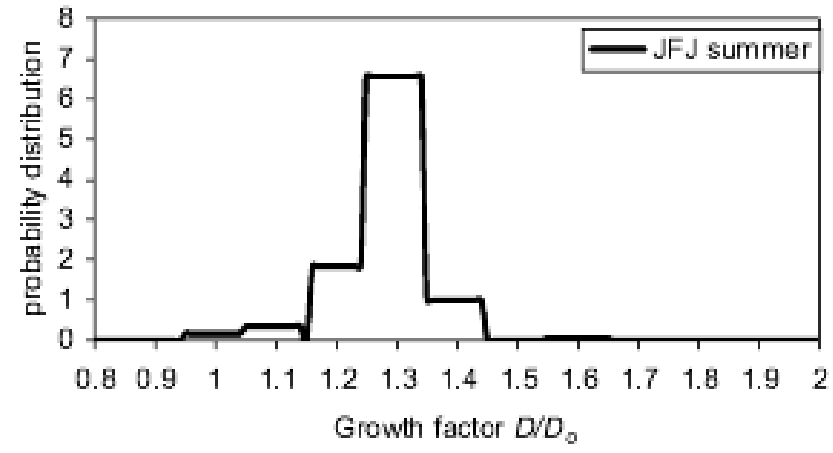

(a)

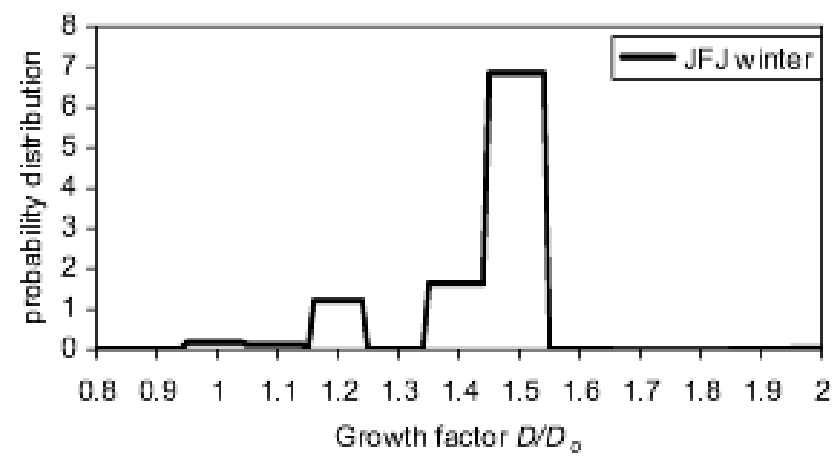

(b)

Fig. 17. Hygroscopic growth factors $G F_{D}$ for $100 \mathrm{~nm}$ particles at the Jungfraujoch in summer (left) and in winter (right).

sured growth factors in the size range $D=50-250 \mathrm{~nm}$ experience a monotonic $7-17 \%$ increase with increasing particle size, indicating that there is a change in the composition in this size range (Weingartner et al., 2002, 2004). At present subtle changes in the composition of particles in the region where the activation cut off for particles is likely to be critical for predicting the indirect effect in aged continental aerosol have not been possible. Whilst knowledge of these subtleties is not required for accurate prediction of the direct effect, the cloud droplet number and hence indirect effect may well be very sensitive to these subtle changes in relative number of Aitken and accumulation mode aerosol and their compositions at sizes equivalent to the typical critical radii of the smallest aerosol particles involved in activation.

Using the individual $G F_{D}$ for the pure inorganic compounds and assuming $G F_{D}$ values at $85 \% R H$ of 1.0 and 1.1 for black carbon and organic carbon, respectively, the measured growth factors were well reproduced by the Zdanovskii-Stokes-Robinson (ZSR) model (Chen et al., 2003) which assumes an ideal mixing behaviour of the chemical species in the aerosol particle. As a consequence of 
this internal mixing, particles do not show efflorescence but rather a continuous increase of $G F_{D}$ with increasing $R H$ (Weingartner et al., 2004), from which it may be inferred that at the JFJ the particles are present as liquid droplets over a broad $R H$ range, consistent with the thermodynamically stable liquid phase of multicomponent organic mixtures (Marcolli et al., 2004).

An analysis of measured number size distributions of interstitial particles and cloud droplet residuals showed that in warm clouds the activated fraction is strongly size dependent. The values with $50 \%$ activation are centred around $D=100 \mathrm{~nm}$, Henning et al. (2002) showing a sharp transition from unactivated to activated particles pointing towards the internal mixing of the sub- $\mu \mathrm{m}$ aerosol. AMS measurements behind the different inlets also show that the organic fraction is scavenged effectively by the cloud. The ratio of cloud droplet number and accumulation mode aerosol number is near unity during clean conditions, and, during more polluted periods the cloud droplet number is considerably less than the accumulation mode number concentration. The mass scavenging ratio of the organic material was shown to be similar to the equivalent inorganic component ratios for any given cloud event. However, variable scavenging ratios of between 0.3 and 1 were observed throughout the experiment, providing an average scavenging ratio of organic material of around 0.5 , generally decreasing with increasing concentrations in given cloud events. Individual case studies also showed that when the organic to inorganic ratio was small the scavenging ratio was larger. Though this may indicate that the organic can inhibit activation, it may be that the ratio reduces with age in the atmosphere and the number scavenging ratio increases on similar timescales. In cases where there was a strong indication of internal mixing, identified by similar mass size modes of the components, the scavenging ratio of the organic material increased substantially. For predominantly warm clouds it was found that black carbon (BC) is scavenged in a very similar way as the total sub$\mu \mathrm{m}$ aerosol volume, indicating that $\mathrm{BC}$ is to a great extent internally mixed. The correlation between the scavenged $\mathrm{BC}$ fraction $\left(S F_{\mathrm{BC}}\right)$ and the activated volume fraction $\left(A F_{V}\right)$ was $S F_{B C}=0.92 \times A F_{V}$ with $R^{2}=0.68$.

Such case studies are not, in themselves, evidence for composition-dependent aerosol effects on cloud activation. The microphysics of the cap clouds at the Jungfraujoch may be dominated by the updraught velocities at cloud base from one event to the next rather than by changes in the aerosol properties. Extended investigations of this type may, however, offer some key insights into the incorporation of organic material and black carbon into cloud.

6.4 Aerosols in the tropical atmosphere: from natural biogenic to biomass burning particles

Aerosol particles in tropical rain forests such as Amazonia and tropical Africa plays a key role in the regional biogeo- chemical cycles (Davidson and Artaxo, 2004), in the radiation balance (Procopio et al., 2003, 2004) and in cloud processes (Andreae et al., 2001, 2002, 2004; Roberts et al., 2002; Silva Dias et al., 2002), There is important interactions between the biosphere and the atmosphere in Amazonia with strong coupling between regional climate, land use, aerosol particles and cloud structure (Silva Dias et al., 2002, 2004). The aerosol in the Amazon basin is dominated throughout the year by organic matter, for both fine and coarse mode, and the particles are mostly soluble in water. The aerosol concentration and composition in Amazonia exhibits strong seasonality, with two very different situations. During the wet season, natural biogenic particles dominate the aerosol population (Artaxo and Hansson, 1995), and in the dry season, biomass burning particles shows very high aerosol loading (Artaxo et al., 1998, 2002).

Aerosol mass concentrations in the wet season is around $10 \mu \mathrm{g} / \mathrm{m}^{3}$, while the number concentration averages about 300 particles/cc. For example, during the clean part of the LB-CLAIRE experiment, particle number concentrations were around 350 particles/cc and $\mathrm{CCN}$ concentrations at $1 \%$ supersaturation were at $267 \pm 132$ particles/cc with $\mathrm{CCN} / \mathrm{CN}$ value was 0.50 at $0.6 \%$ supersaturation, rising to 0.71 at $1.0 \%$ supersaturation (Roberts et al., 2000, 2001). The sub- $\mu \mathrm{m}$ aerosol particle size distribution was bimodal (Aitken and accumulation mode with geometric mean around $65 \mathrm{~nm}$ and $120 \mathrm{~nm}$ respectively) for most of the time. A low diameter growth factor of 1.3 taken from dry to $90 \% \mathrm{RH}$ was measured for $100 \mathrm{~nm}$ particles (Rissler et al., 2004). Around 80\% of the biogenic aerosol mass is in the coarse mode. In the fine mode, the particles are dominated by organic aerosols from gas-to particle conversion of biogenic VOCs emitted by the vegetation. Organic compounds are responsible for $70 \%$ of the aerosol mass, with very low sulphate and nitrate concentration (Graham et al., 2003a,b). Soil dust is a minor component (about $10-20 \%$ in mass, with sporadic impact from long range transport of Sahara dust aerosols in Amazonia (Formenti et al., 2001; Artaxo et al., 1998). Recently, Claeys et al. (2004) have demonstrated the possibility that oxidation products of isoprene could make a fraction of natural wet season aerosol particles in considerable amounts. Roberts et al. (2002) analyzed the effect of sulfur compounds from decaying organic matter deposited on pre-existing particles, enhancing their capacity to become $\mathrm{CCN}$, showing that the amount of sulfate on the particle was directly related to the activation fraction of the aerosol for a given supersaturation.

In the dry season, biomass burning aerosol particles dominates the atmosphere, with PM2.5 as high as $600 \mu \mathrm{g} / \mathrm{m}^{3}$, and particle number concentrations of around 30000 particles/cc (Artaxo et al., 1998, 2002; Andreae et al., 2002). Fine mode aerosol comprises $90 \%$ of the aerosol mass, and organic compounds are responsible for around $70 \%$ of the aerosol mass (Mayol-Bracero et al., 2002; Graham et al., 2003a). The Large Scale Biosphere-Atmosphere Experiment in Amazonia - Smoke, Aerosols, Clouds, Rainfall and Climate 
(LBA-SMOCC) experiment was conducted in the Amazon Basin, with the principal purpose of investigating how and to what extent aerosol particles produced by biomass burning alter cloud formation. Andreae et al. (2004) showed the large impact of pyrogenic particles in cloud properties during the biomass burning season, with large changes in cloud microphysics from natural conditions to heavy biomass burning impacts. Heavy smoke from forest fires in the Amazon was observed to reduce cloud droplet size and so delay the onset of precipitation from $1.5 \mathrm{~km}$ above cloud base in pristine clouds to more than $5 \mathrm{~km}$ in polluted clouds and more than $7 \mathrm{~km}$ in pyro-clouds. The average cloud depth required for the onset of warm rain increased by $\sim 350 \mathrm{~m}$ for each additional $100 \mathrm{CCN} / \mathrm{cc}$ at a super-saturation of $0.5 \%$ (Freud et al., 2005). Using remote sensing techniques, Koren et al. (2004) demonstrated that no low clouds were observed in areas with enough biomass burning smoke to create an aerosol optical thickness above 1.5 at $500 \mathrm{~nm}$. This result serves as a reminder that, although they are beyond the scope of this review, dynamical responses to aerosol light scattering and absorption can lead very important effects on clouds. Modelling of this system by Feingold et al. (2005) using large eddy simulations suggests that the primary reason for reduction in cloud fraction is the suppression of surface fluxes by the smoke.

It is becoming clear that larger aerosol loadings in the atmosphere cause changes in cloud microphysics, with both in situ and satellite measurements indicating smaller droplet sizes with increasing aerosol loading and changes in cloud dynamics. However, the final effect on the precipitation rate and amount is still unclear. For example, delays in the onset of precipitation do not necessarily translate into less precipitation over the lifetime of a storm. Several researchers have looked at possible changes in the precipitation rate with large temporal or spatial changes in aerosols, but there is no clear signal on this critical issue. This area needs attention due to possible changes in precipitation rates and amounts. Additionally, the quantitative aspects of the aerosol indirect effects, as well as the semi-indirect effect, is still in its infancy, with potentially significant changes in the global and regional atmospheric radiation balance (Silva Dias et al., 2004).

\section{Climate model representation of aerosol effects on cloud activation}

GCMs are being used to examine the influence of aerosols and clouds on global climate. This is a priori an enormous challenge given the need for GCMs to integrate myriad atmospheric, land, and ocean processes on a broad range of temporal and spatial scales. It may be argued that GCMs need to at least represent the collocation of aerosol and clouds in a statistically representative manner if they are to be relied upon to assess the aerosol indirect effect. As discussed earlier, convection and cloud formation are poorly resolved in these models. GCMs have grid spacings on the order of $100 \mathrm{~km}$ and clouds often fill only a small fraction of the gridcell area. Cloud scale updraughts, which drive cloud supersaturations and the fraction of activated particles, are also poorly resolved. GCM-grid resolved vertical velocities are very small (order $1 \mathrm{~cm} \mathrm{~s}^{-1}$ ) and do not represent the cloudscale vertical velocities (order $1 \mathrm{~ms}^{-1}$ ). Detailed treatment of aerosol-cloud interactions in GCMs thus relies heavily on the GCM subgrid parameterizations of convection and cloudscale updraughts, growth of drops (and ice particles), and precipitation. Thus detailed representation of the aerosol size distribution and composition of the kind described in this paper is a necessary, but not sufficient condition for assessments of aerosol indirect effects using GCMs. This being said, GCMs are an essential climate prediction tool and introduction of physically-based treatments of aerosol, clouds, and precipitation is crucial given our current limited understanding of the potential effects on climate change.

\subsection{Current approaches to represent aerosols in global cli- mate models}

Since the pioneering study by Langner and Rodhe (1991), who used a coarse horizontal resolution chemical transport model based on climatological meteorology for the simulation of the sulphur cycle, there have been steady advances in the treatment of the complexity of the aerosol precursor chemistry, transport processes, parameterisation of particle dry deposition, and wet removal. Recently, attempts have been undertaken to calculate not just the aerosol mass, but also the particle number concentration by parameterising aerosol formation and dynamical processes. Two kinds of aerosol dynamics models have been developed: modal schemes and bin schemes. Modal schemes have been applied for mineral dust (Schulz et al., 1998) and for sulphuric acid, soot and seasalt (Wilson et al., 2001). Bin schemes have been applied for sulphate (Adams and Seinfeld, 2002), for mineral dust studies (Tegen and Fung, 1994), for sea salt aerosols (Gong et al., 1997) and for sulphate and seasalt (Gong and Barrie, 2003; Spracklen et al., 2005).

Most of the earlier studies concerned with the effect of aerosol particles on the climate system have taken sulphate particles as a surrogate for all anthropogenic aerosols (Boucher and Lohmann, 1995; Jones et al., 1994). Lately most major GCMs also include carbonaceous aerosols, dust and sea salt (for state of model development see: AEROCOM model intercomparison: http://nansen.ipsl.jussieu.fr/ AEROCOM/ and Kinne et al., 2003). Simulating nitrate aerosols is more difficult, because of their semi-volatile nature (Adams et al., 2001). Aside from physical and microphysical processes the lack of time-resolved and accurate emission inventories introduces large uncertainties (Bond et al., 2004). In particular, biogenic sources and emissions from biomass burning are highly uncertain. Both biogenic and biomass burning emissions depend on environmental 
conditions (e.g. weather) and exhibit high interannual variability, which has not been taken into account by climate studies. Probably the largest uncertainty is associated with organic aerosols because current measurement techniques cannot identify all organic species (Kanakidou et al., 2005).

There is increasing evidence that aerosol particles are predominantly a conglomerate of different internally mixed chemical substances (see Sects. 3.1 and 3.2). In contrast most GCMs still treat aerosols as external mixtures in terms of their optical properties (e.g. Feichter et al., 1997) because internal mixtures have more degrees of freedom, are more complex, and require an added computational burden. Advanced aerosol modules in GCMs have been expanded to include aerosol mixtures and to predict aerosol number as well as mass, so that processes that influence aerosol mass only do not affect aerosol number, and processes that influence aerosol number only do not affect aerosol mass for each mode (Ghan et al., 2001; Zhang et al., 2002b; Stier et al., 2005).

7.2 Current approaches to represent aerosol-cloud interactions in global climate models

Activation of aerosol particles to form cloud droplets is one of the weakest links in estimates of the indirect aerosol effects. In order to treat cloud droplet formation accurately, the aerosol number concentration, its chemical composition and the vertical velocity on the cloud scale need to be known. Abdul-Razzak and Ghan (2000) developed a parameterisation based on Köhler theory that can describe cloud droplet formation for a multi-modal aerosol. Also, the competition between different aerosol species, such as sulphate and sea salt, (Ghan et al., 1998; O'Dowd et al., 1999) has recently been considered (Ghan et al., 2001). Nenes and Seinfeld (2003) derived a largely analytical solution to the equations for droplet activation. Their sectional treatment treats externally mixed aerosol of variable composition and includes kinetic growth limitations, and surface active species.

As discussed in Sect. 4, chemical effects of the same order as the indirect effect were pointed out by Nenes et al. (2002) and as large as unresolved cloud dynamics by Lance et al. (2004). Thus, they need to be included in GCMs as well. The effect of surface-active organics and slightly soluble organics has recently been included in the parameterisation of cloud droplet formation by Abdul-Razzak and Ghan (2004, 2005). Other effects of organics, such as their film-forming ability are treated by Nenes and Seinfeld (2003) and Fountoukis and Nenes (2005). In order to apply one of these parameterisations, the updraught velocity relevant for cloud formation needs to be known. Some GCMs apply a Gaussian distribution (Chuang et al., 1997) or use the turbulent kinetic energy as a surrogate for it (Lohmann et al., 1999). Other GCMs avoid this issue completely and use empirical relationships between aerosol mass and cloud droplet number concentration instead (Menon et al., 2002). This method is limited because of a scarcity of observational data. At present, relationships can only be derived between sulphate aerosols, sea salt, organic carbon and cloud droplet number, but no concurrent data for dust or black carbon and cloud droplet number are available yet.

\subsection{What is the extent of predicted aerosol effect?}

Some attempts have been made to estimate the total radiative forcing since pre-industrial times including all quantitative radiative forcing estimates and uncertainties included in the IPCC 2001 bar chart (Boucher and Haywood, 2001). The resulting total radiative forcing (well-mixed greenhouse gases, solar activity, ozone, direct aerosol effects and Twomey effect) has a $75-97 \%$ probability of being positive. Note that the Twomey effect is the only aerosol effect that can be considered a forcing because it can be calculated from two radiative transfer calculations where the only difference is the aerosol-induced increase in cloud droplet number for a constant liquid water path. These estimates neglect the cloud lifetime effect and aerosol effects on mixed-phase and ice clouds because our knowledge of these effects is not yet sufficient to predict their magnitudes and because all other aerosol effects include feedbacks and interactions within the climate system. Probability ranges, however, can be estimated from inverse simulations or thermodynamic considerations (Anderson et al., 2003; Crutzen and Ramanathan, 2003). They limit the sum of all indirect aerosol effect to between 0 and $-2 \mathrm{Wm}^{-2}$. Given that the GCM estimates for the Twomey effect alone amount to -0.5 to $-1.9 \mathrm{~W} \mathrm{~m}^{-2}$ (Fig. 18 and Lohmann and Feichter, 2005), either all other aerosol indirect effects cancel each other or the Twomey effect is smaller than current climate models predict.

Thus uncertainties in aerosol forcing must be reduced at least three-fold for uncertainties in climate sensitivities to be meaningfully reduced and bounded (Schwartz, 2004). One major problem for assessing the aerosol indirect effect is its large regional variability (Ramanathan, 2001; Chameides et al., 2002). Thus, it is questionable whether the global mean top-of-the atmosphere forcing is sufficient to characterize the radiative impact of aerosols and the aerosol effects on the hydrological cycle.

\section{Conclusions, future directions and suggestions}

Atmospheric aerosol size distributions are highly variable and both the number of particles in a given size range and the gradient of the distribution in certain critical size ranges will determine activation behaviour moving into supersaturation. Size distribution characteristics strongly interact with the dynamics to determine the number of activated droplets and these interactions are dependent on the composition distribution. The sensitivities of cloud droplet size and number to the aerosol size, composition and updraught are not equal 

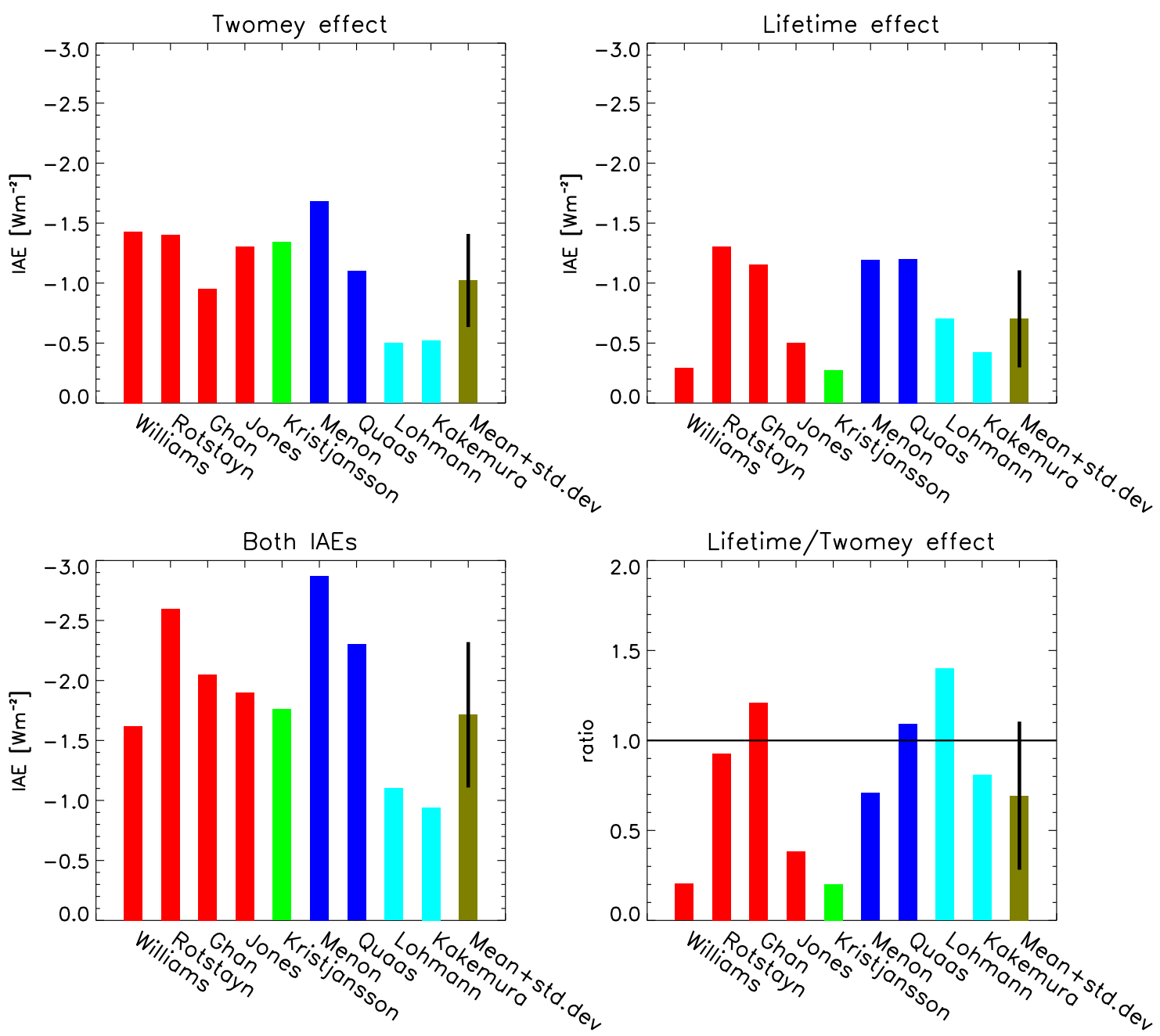

Fig. 18. Global mean Twomey effect, lifetime effect, both effect and the ratio lifetime effect/Twomey effect of anthropogenic sulfate aerosols (red bars) from Williams et al. (2001); Rotstayn and Penner (2001); Ghan et al. (2001); Jones et al. (2001), of anthropogenic sulfate and black carbon (green bars) from Kristjansson (2002), of anthropogenic sulfate and organic carbon (blue bars) from Menon et al. (2002) and Quaas et al. (2004), of anthropogenic sulfate and black, and organic carbon (turquoise bars) from Lohmann et al. (2000) and Takemura et al. (2005) and the mean plus standard deviation from all simulations (olive bars). The results from Menon et al. (2002) and Ghan et al. (2001) are taken to be the averages of the simulations for only the Twomey effect and for both effects. This figure has been taken from Lohmann and Feichter (2005).

and are interdependent, though in all cases the aerosol number appears important.

Inorganic electrolyte components of atmospheric aerosols are readily hygroscopic and evidence suggests that organic components contribute to growth of aged particles, but only with a moderate growth factor of $\sim 1.15$ at $90 \% R H$. The accumulation mode particles are generally more hygroscopic than Aitken mode particles. With the exception of urban areas, most environments are dominated by particles which readily take up at least a moderate amount of water. There is some trend of increasing fraction of readily hygroscopic particles, increasing growth factors and decreasing growth spread from anthropogenically influenced to remote areas.
External mixtures of particle modes with respect to water uptake and growth spread within modes (quasi-internally mixed) are nearly ubiquitous.

Accumulation mode particles are generally more hygroscopic than Aitken mode particles. This is an important issue because the minimum size of particles activated to form cloud droplets is often near the border between these two particle modes.

\subsection{Measurement requirements}

A range of offline and online techniques are available to measure size segregated aerosol composition. No single technique is capable of providing all required information to 
enable prediction of cloud activation; though a combination of techniques can deliver useful highly time and spatiallyresolved chemical composition and mixing state information which may be used as input to cloud parcel models. Impactor sampling and offline analysis may be used to derive model compounds to represent the organic aerosol components by mass. Higher size and time resolution of component mass loading down to activable sizes may be provided by AMS ensemble distributions; but with little organic component discrimination. Single particle instruments can provide component mixing state and broad composition by number down to near the activation threshold dry size. The actual measurement requirement for estimation of the aerosol effect on droplet activation is size-resolved chemical and physical property data for particles in the 40 to $200 \mathrm{~nm}$ diameter range.

Given the complexity of organic components, it is unlikely that a detailed understanding of their effects on surface tension, uptake kinetics and equilibrium water content will be readily and reliably predictable from composition measurements alone; hence direct measurements of physical properties are also required at the sizes near the cloud activation threshold. Two such useful derived properties are the hygroscopic growth factor and the CCN activation spectra. Direct surface tension measurements are also useful.

A comparison of activation spectra between those measured in $\mathrm{CCN}$ instruments and those derived from other measured properties such as composition may provide reliable data sets for cloud model initialisation and reveal limitations of the measurements. The duration of particle exposure to the maximum supersaturation in such measurements should mimic that which occurs during activation in an updraft. Laboratory experiments conducted with flow-through cloud simulators (Stratmann et al., 2004) together with fielddeployable aerosol and CCN instrumentation should be encouraged. The proposed studies could provide important guidance on several of the outstanding issues addressed here, including the effects of humidity preconditioning on aerosol activation, the reliability of field-deployable CCN instrumentation and effects of complex aerosol composition on activation.

\subsection{Effect clarification}

In Sect. 4 we considered the possible effects of component properties on cloud activation. Several concrete recommendations follow from this consideration:

i) The effect of limited component solubility alone is shown to be of limited relevance to real multicomponent atmospheric aerosol activation,

ii) The degree to which a surfactant partitions between the bulk and the surface of the droplet is dependent on the nature of the dominant surface active components and surfactant partitioning may need to be considered in cloud modelling studies. Simply accounting for surface tension reduction by calculating the surfactant concentration from its total mass in the droplet will lead to underestimation of the critical supersaturation in some cases. It should be noted that solely reducing the surface tension would worsen the overprediction of $\mathrm{CCN}$ and droplet numbers commonly found in closure studies (Sect. 5),

iii) If surfactant films are present, they are probably more likely to be in the expanded than condensed state and are unlikely to influence hygroscopic or $\mathrm{CCN}$ properties greatly by mass transfer limitation,

iv) It is therefore critically important to clarify whether ordered organic films truly do exist on growing cloud droplets, whether surface partitioning effects are significant or whether surface tension suppression predominantly results from WSOC. Surrogates for representing atmospheric aerosol should therefore be chosen with caution,

v) Cloud model studies have shown that the effects of nitric acid and other soluble trace gases on droplet activation depend heavily on the kinetics of cloud formation and cannot be explained with an equilibrium Köhler theory alone (see dynamical considerations, below),

vi) Use of the primitive form of the Köhler equation can circumvent requirement for unknown composition related parameters, but require either laboratory-determined or predicted water activities, and surface tensions. Approaches using such techniques may prove valuable for multicomponent droplet activation prediction.

\subsection{Dynamical considerations}

The complexity of aerosol composition suggests potential effects on cloud droplet activation that are very large based on Köhler theory, and yet these very large differences are not realised when considering the dynamical system of a population of aerosol growing and competing for water vapour (Phinney et al., 2003; Ming and Russell, 2004; Ervens et al., 2005). Composition effects are significantly reduced by feedbacks in the dynamical system, particularly under low aerosol loadings. The potential for composition effects to be realised is greatest under polluted conditions with low water vapour supply (Nenes et al., 2002; Lance et al., 2004). The effects of kinetic limitation on droplet activation are significant, all else being equal: smaller uptake resulting in larger activated fractions. The natural variability in aerosol size distributions can generate conditions under which increases in aerosol do not necessarily translate to increases in drop concentration. A case in point is the role of large or giant aerosol in limiting cloud supersaturation, thus wielding influence beyond that expected by their numbers on the activation 
of small particles (Ghan et al., 1998; O'Dowd et al., 1999; Feingold, 2001). Coupled growth models are required to assess these effects and greatest deviation of such predictions from equilibrium tend to be strongest for the small particles where composition effects are most important.

\subsection{Synthesising system complexity}

It was shown in Sect. 6 that the complexity of aerosol behaviour varies significantly between locations; how cloud activation behaviour is affected by this complexity must be established to enable such considerations to be captured in large-scale models. Section 7 provided a discussion of the current descriptions of aerosol and aerosol-cloud interactions in global models, a discussion of the difficulties in such approaches and a summary of the range of predicted radiative forcing based on these treatments. In spite of our very best efforts at explaining the relationship between aerosol size/composition and drop size distributions, we are inherently limited in our current climate predictive capabilities by the difficulty of resolving convection and clouds in GCMs. Marine boundary layer clouds, such as stratocumulus, present a challenge because they are not well resolved in GCMs, and their contrast with the dark underlying ocean represents a significant climate forcing.

Whilst it is evident that there is significant complexity in ambient aerosol composition and behaviour, it is crucial to evaluate the extent to which details of aerosol-cloud interactions are important for the overall understanding of their impact on the climate system. Useful questions that should be posed include:

i) is detailed aerosol size distribution and composition always required or are there adequate proxy that are easier to perform or representations that are easier to use? For example, how closely can the effects of composition be described simply with a hygroscopic growth factor or insoluble fraction in addition to the size distributions?

ii) How many of the complex interactions in a particular system are important, and at what spatial/temporal scales?

iii) Can we synthesise our understanding of the complexity into physically-based parameterisations that capture the essence of the process under consideration?

iv) Will such parameterisations represent adequately the underlying physics when included in global scale models?

\subsection{Addressing temporal/spatial scales}

An important issue for both observations and models is the question of scale. Current climate models do not incorporate the small temporal/spatial scales needed for adequate resolution of many processes; aerosol-microphysics-chemistry processes are a case in point. Predicting the impact of the aerosol indirect effect is an enormous challenge because it requires that models correctly predict the collocation and timing of aerosol and cloud events, as well as the interactions between aerosol and clouds at small scales.

Observations and modeling should, as a first step, be performed at the scale appropriate to the process or interaction under investigation. Observations and model output need to be compared at similar scales, starting from the smallest scales pertinent to the process, and ranging up to the regional and global scales. A methodology for consistent transfer of understanding and representation of processes from the smallest to largest scales needs to be developed. This could consist of embedding detailed models in climate models based on the principles of synthesis and parameterisation outlined above, or increasing the spatial and temporal resolution of the climate models to resolve the processes themselves.

Acknowledgements. This publication resulted from discussions held during the January 2005 Speciality Conference on the Indirect Effect of Aerosols on Climate, in Manchester, England, which was jointly sponsored by the International Global Atmospheric Chemistry Project (IGAC; A core project of the International Geosphere Biosphere Programme, IGBP) and by the US NOAA and NASA programmes. Support was also provided by the European Network of Excellence on Atmospheric Composition Change, ACCENT. This work was partially supported by the European Commission (Projects QUEST and PHOENICS and SMOCC), the Italian Ministry of Environment (Italy-USA Cooperation on Science and Technology of Climate Change), by the Academy of Finland (Center of Excellence Program) and by the UK Natural Environment Research Council. We'd also like to thank R. Sorjamaa for the calculations illustrated in, and preparation of, Fig. 10.

Edited by: A. Nenes

\section{References}

Abdul-Razzak, H. and Ghan, S. J.: A parameterization of aerosol activation: 2. Multiple aerosol types, J. Geophys. Res.-Atmos., 105, 6837-6844, 2000.

Abdul-Razzak, H. and Ghan, S. J.: Parameterization of the influence of organic surfactants on aerosol activation, J. Geophys. Res.-Atmos., 109, doi:10.1029/2003JD004043, 2004.

Abdul-Razzak, H. and Ghan, S. J.: Influence of slightly soluble organics on aerosol activation, J. Geophys. Res.-Atmos., 110, D06206, doi:10.1029/2004JD005324, 2005.

Ackerman, A. S., Toon, O. B., Taylor, J. P., Johnson, D. W., Hobbs, P. V., and Ferek, R. J.: Effects of aerosols on cloud albedo: Evaluation of Twomey's parameterization of cloud susceptibility using measurements of ship tracks, J. Atmos. Sci., 57, 2684-2695, 2000.

Adams, P. J. and Seinfeld, J. H.: Predicting global aerosol size distributions in general circulation models, J. Geophys. Res.Atmos., 107, 4370, doi:10.1029/2001JD001010, 2002. 
Adams, P. J., Seinfeld, J. H., Koch, D., Mickley, L., and Jacob, D.: General circulation model assessment of direct radiative forcing by the sulfate-nitrate-ammonium-water inorganic aerosol system, J. Geophys. Res.-Atmos., 106, 1097-1111, 2001.

Aklilu, Y. A. and Mozurkewich, M.: Determination of external and internal mixing of organic and inorganic aerosol components from hygroscopic properties of submicrometer particles during a field study in the Lower Fraser Valley, Aerosol Sci. Technol., 38, 140-154, 2004.

Albrecht, B. A.: Aerosols, Cloud Microphysics, and Fractional Cloudiness, Science, 245, 1227-1230, 1989.

Alfarra, M. R., Coe, H., Allan, J. D., Bower, K. N., Boudries, H., Canagaratna, M. R., Jimenez, J. L., Jayne, J. T., Garforth, A. A., Li, S. M., and Worsnop, D. R.: Characterization of urban and rural organic particulate in the lower Fraser valley using two aerodyne aerosol mass spectrometers, Atmos. Environ., 38, 57455758, 2004.

Allan, J. D., Alfarra, M. R., Bower, K. N., Williams, P. I., Gallagher, M. W., Jimenez, J. L., McDonald, A. G., Nemitz, E., Canagaratna, M. R., Jayne, J. T., Coe, H., and Worsnop, D. R.: Quantitative sampling using an Aerodyne aerosol mass spectrometer: 2. Measurements of fine particulate chemical composition in two UK cities, J. Geophys. Res.-Atmos., 108, 4284, doi:10.1029/2002JD002359, 2003a.

Allan, J. D., Jimenez, J. L., Williams, P. I., Alfarra, M. R., Bower, K. N., Jayne, J. T., Coe, H., and Worsnop, D. R.: Quantitative sampling using an Aerodyne aerosol mass spectrometer, 1. Techniques of data interpretation and error analysis, J. Geophys. Res.Atmos., 108, 4283, doi:10.1029/2002JD002358, 2003 b.

Allan, J. D., Delia, A. E., Coe, H., Bower, K. N., Alfarra, M. R., Jimenez, J. L., Middlebrook, A. M., Drewnick, F., Onasch, T. B., Canagaratna, M. R., Jayne, J. T., and Worsnopf, D. R.: A generalised method for the extraction of chemically resolved mass spectra from aerodyne aerosol mass spectrometer data, J. Aerosol Sci., 35, 909-922, 2004.

Alofs, D. J., Trueblood, M. B., White, D. W., and Behr, V. L.: Nucleation experiments with monodisperse $\mathrm{NaCl}$ aerosols, J. Appl. Meteorol., 18, 1106-1117, 1979.

Anderson, T. L., Charlson, R. J., Schwartz, S. E., Knutti, R., Boucher, O., Rodhe, H., and Heintzenberg, J.: Climate forcing by aerosols - a hazy picture, Science, 300, 1103-1104, 2003.

Andreae, M. O., Artaxo, P., Fischer, H., Freitas, S. R., Gregoire, J. M., Hansel, A., Hoor, P., Kormann, R., Krejci, R., Lange, L., Lelieveld, J., Lindinger, W., Longo, K., Peters, W., de Reus, M., Scheeren, B., Dias, M., Strom, J., van Velthoven, P. F. J., and Williams, J.: Transport of biomass burning smoke to the upper troposphere by deep convection in the equatorial region, Geophys. Res. Lett., 28, 951-954, 2001.

Andreae, M. O., Artaxo, P., Brandao, C., Carswell, F. E., Ciccioli, P., da Costa, A. L., Culf, A. D., Esteves, J. L., Gash, J. H. C., Grace, J., Kabat, P., Lelieveld, J., Malhi, Y., Manzi, A. O., Meixner, F. X., Nobre, A. D., Nobre, C., Ruivo, M., Silva-Dias, M. A., Stefani, P., Valentini, R., von Jouanne, J., and Waterloo, M. J.: Biogeochemical cycling of carbon, water, energy, trace gases, and aerosols in Amazonia: The LBAEUSTACH experiments, J. Geophys. Res.-Atmos., 107, 8066, doi:10.1029/2001JD000524, 2002.

Andreae, M. O., Rosenfeld, D., Artaxo, P., Costa, A. A., Frank, G. P., Longo, K. M., and Silva-Dias, M. A. F.: Smoking rain clouds over the Amazon, Science, 303, 1337-1342, 2004.

Ansari, A. S. and Pandis, S. N.: Water absorption by secondary organic aerosol and its effect an inorganic aerosol behavior, Environ. Sci. Technol., 34, 71-77, 2000.

Artaxo, P. and Hansson, H. C.: Size Distribution of Biogenic Aerosol-Particles from the Amazon Basin, Atmos. Environ., 29, 393-402, 1995.

Artaxo, P., Fernandes, E. T., Martins, J. V., Yamasoe, M. A., Hobbs, P. V., Maenhaut, W., Longo, K. M., and Castanho, A.: Largescale aerosol source apportionment in Amazonia, J. Geophys. Res.-Atmos., 103, 31 837-31 847, 1998.

Artaxo, P., Martins, J. V., Yamasoe, M. A., Procopio, A. S., Pauliquevis, T. M., Andreae, M. O., Guyon, P., Gatti, L. V., and Leal, A. M. C.: Physical and chemical properties of aerosols in the wet and dry seasons in Rondonia, Amazonia, J. Geophys. Res.-Atmos., 107, 2002.

Baltensperger, U., Streit, N., Weingartner, E., Nyeki, S., Prevot, A. S. H., van Dingenen, R., Virkkula, A., Putaud, J.-P., Even, A., ten Brink, H., Blatter, A., Neftel, A., and Gaggeler, H. W.: Urban and rural aerosol characterization of summer smog events during the PIPAPO field campaign in Milan, Italy, J. Geophys. Res., 107(1-13), 8193, doi:10.1029/2001JD001292, 2002.

Baltensperger, U., Kalberer, M., Dommen, J., Paulsen, D., Alfarra, M. R., Coe, H., Fisseha, R., Gascho, A., Gysel, M., Nyeki, S., Sax, M., Steinbacher, M., Prevot, A. S. H., Sjogren, S., Weingartner, E., and Zenobi, R.: Secondary organic aerosols from anthropogenic and biogenic precursors, Faraday Discuss., 130, 265-278, 2005.

Bahreini, R., Jimenez, J. L., Wang, J., Flagan, R. C., Seinfeld, J. H., Jayne, J. T., and Worsnop, D. R.: Aircraft-based aerosol size and composition measurements during ACE-Asia using an Aerodyne aerosol mass spectrometer, J. Geophys. Res., 108, 8645, 2003.

Barnes, G. T.: The Effects of Monolayers on the evaporation of liquids, Advances in Colloid Interface Science, 25, 89-200, 1986.

Bates, J. R.: A dynamical stabilizer in the climate system: a mechanism suggested by a simple model, Tellus, Series A, 51A, 349372, 1999.

Berg, O. H., Swietlicki, E., Frank, G., Martinsson, B. G., Cederfelt, S. I., Laj, P., Ricci, L., Berner, A., Dusek, U., Galambos, Z., Mesfin, N. S., Yuskiewicz, B., Wiedensohler, A., Stratmann, F. and Orsini, D.: Comparison of observed and modeled hygroscopic behavior of atmospheric particles, Contr. Atmos. Phys., 71, 47-64, 1998a.

Berg, O. H., Swietlicki, E., and Krejci, R.: Hygroscopic growth of aerosol particles in the marine boundary layer over the $\mathrm{Pa}$ cific and Southern Oceans during the First Aerosol Characterization Experiment (ACE 1), J. Geophys. Res., 103, 16 535-16545, $1998 b$.

Bianco, H. and Marmur, A.: The Dependence of the SurfaceTension of Surfactant Solutions on Drop Size, Journal of Colloid and Interface Science, 151, 517-522, 1992.

Bigg, E. K.: Discrepancy between observation and prediction of concentrations of cloud condensation nuclei, Atmos. Res., 20, 82-86, 1986.

Bigg, E. K. and Leck, C.: Cloud-active particles over the central Arctic Ocean, J. Geophys. Res.-Atmos., 106, 32 155-32 166, 2001.

Bigg, E. K., Gras, J. L. and Evans, C.: Origin of Aitken Particles in Remote Regions of the Southern-Hemisphere, J. Atmos. Chem., 
1, 203-214, 1984.

Bigg, E. K., Gras, J. L., and Mossop, D. J. C.: Wind-Produced Submicron Particles in the Marine Atmosphere, Atmos. Res., 36, 55-68, 1995.

Bilde, M. and Svenningsson, B.: CCN activation of slightly soluble organics: the importance of small amounts of inorganic salt and particle phase, Tellus Series B - Chemical and Physical Meteorology, 56, 128-134, 2004.

Bilde, M., Svenningsson, B., Monster, J., and Rosenorn, T.: Evenodd alternation of evaporation rates and vapor pressures of C3C9 dicarboxylic acid aerosols, Environ. Sci. Technol., 37, 13711378, 2003.

Bond, T. C., Streets, D. G., Yarber, K. F., Nelson, S. M., Woo, J. H., and Klimont, Z.: A technology-based global inventory of black and organic carbon emissions from combustion, J. Geophys. Res.-Atmos., 109, 14 203, doi:10.1029/2003JD003697, 2004.

Boucher, O. and Haywood, J.: On summing the components of radiative forcing of climate change, Climate Dynamics, 18, 297302, 2001.

Boucher, O. and Lohmann, U.: The Sulfate-CCN-Cloud Albedo Effect - a Sensitivity Study with 2 General-Circulation Models, Tellus Series B - Chemical and Physical Meteorology, 47, 281300, 1995.

Boudries, H., Canagaratna, M. R., Jayne, J. T., Alfarra, M. R., Allan, J., Bower, K. N., Coe, H., Pryor, S. C., Jimenez, J. L., Brook, J. R., Li, S., and Worsnop, D. R.: Chemical and physical processes controlling the distribution of aerosols in the Lower Fraser Valley, Canada, during the Pacific 2001 field campaign, Atmos. Environ., 38, 5759-5774, 2004.

Boy, M., Rannik, U., Lehtinen, K. E. J., Tarvainen, V., Hakola, H., and Kulmala, M.: Nucleation events in the continental boundary layer: Long-term statistical analyses of aerosol relevant characteristics, J. Geophys. Res., 108(D21), 4667, doi:10.1029/2003JD003838, 2003

Brenguier, J.-L.: Introduction to Special Section: An experimental study of the aerosol indirect effect for validation of climate model parameterizations, J. Geophys. Res., 108, 8627, doi:10.1029/2003JD003849, 2003.

Brimblecombe, P. and Latif, M. T.: Rediscovering Atmospheric Surfactants, Environ. Chem., 1, 11-12, doi:10.1071/EN04044, 2004.

Broekhuizen, K., Kumar, P. P., and Abbatt, J. P. D.: Partially soluble organics as cloud condensation nuclei: Role of trace soluble and surface active species, Geophys. Res. Lett., 31, L01 107, doi:10.1029/2003GL018203, 2004.

Brooks, S. D., DeMott, P. J., and Kreidenweis, S. M.: Water uptake by particles containing humic materials and mixtures of humic materials with ammonium sulfate, Atmos. Environ., 38, 18591868, 2004.

Burnet, F. and Brenguier, J.-L.: Comparison between Standard and Modified Forward Scattering Spectrometer Probes during the Small Cumulus Microphysics Study, J. Atmos. Oceanic Technol., 19, 1516-1531, 2002.

Busch, B., Kandler, K., Schutz, L., and Neususs, C.: Hygroscopic properties and water-soluble volume fraction of atmospheric particles in the diameter range from $50 \mathrm{~nm}$ to $3.8 \mu \mathrm{m}$ during LACE 98, J. Geophys. Res., 107(D21), 8119, doi:10.1029/2000JD000228, 2002.

Cabada, J. C., Rees, S., Takahama, S., Khlystov, A., Pandis, S. N.,
Davidson, C. I., and Robinson, A. L.: Mass size distributions and size resolved chemical composition of fine particulate matter at the Pittsburgh supersite, Atmos. Environ., 38, 3127-3141, 2004.

Canagaratna, M. R., Jayne, J. T., Ghertner, D. A., Herndon, S., Shi, Q., Jimenez, J. L., Silva, P. J., Williams, P., Lanni, T., Drewnick, F., Demerjian, K. L., Kolb, C. E., and Worsnop, D. R.: Chase studies of particulate emissions from in-use New York City vehicles, Aerosol Sci. Technol., 38, 555-573, 2004.

Cantrell, W., Shaw, G., Cass, G. R., Chowdhury, Z., Hughes, L. S., Prather, K. A., Guazzotti, S. A., and Coffee, K. R.: Closure between aerosol particles and cloud condensation nuclei at Kaashidhoo Climate Observatory, J. Geophys. Res., 106, 28711-28 718, 2001.

Carrico, C. M., Kreidenweis, S. M., Malm, W. C., Day, D. E., Lee, T., Carrillo, J., McMeeking, G. R., and Collett, J. L.: Hygroscopic growth behavior of a carbon-dominated aerosol in Yosemite National Park, Atmos. Environ., 39, 1393-1404, 2005.

Carvalho, A., Pio, C., and Santos, C.: Water-soluble hydroxylated organic compounds in German and Finnish aerosols, Atmos. Environ., 37, 1775-1783, 2003.

Cavalli, F., Facchini, M. C., Decesari, S., Emblico, L., Mircea, M., Fuzzi, S., and Mancinelli, V.: Size-segregated chemical composition of aerosol particle at Hyytiälä, Finland, submitted for publication in Report Series Aerosol Science, Finish Association for Aerosol Research, Helsinki, 2004a.

Cavalli, F., Facchini, M. C., Decesari, S., Mircea, M., Emblico, L., Fuzzi, S., Ceburnis, D., Yoon, Y. J., O’Dowd, C. D., Putaud, J. P., and Dell'Acqua, A.: Advances in characterization of size-resolved organic matter in marine aerosol over the North Atlantic, J. Geophys. Res.-Atmos., 109, d24215, doi:10.1029/2004JD005137, 2004b.

Chameides, W. L., Lou, C., Saylor, R., Streets, D., Huang, Y., Bergin, M., and Giorgi, F.: Correlation between modelcalculated anthropogenic aerosols and satellite-derived cloud optical depths: Indication of indirect effect?, J. Geophys. Res.Atmos., 107, 4085, doi:10.1029/JD000208, 2002.

Chan, M. N. and Chan, C. K.: Mass transfer effects in hygroscopic measurements of aerosol particles, Atmos. Chem. Phys., 5, 2703-2712, 2005, http://www.atmos-chem-phys.net/5/2703/2005/.

Chan, Y. C., Simpson, R. W., McTainsh, G. H., Vowles, P. D., Cohen, D. D., and Bailey, G. M.: Source apportionment of PM2.5 and PM10 aerosols in Brisbane (Australia) by receptor modelling, Atmos. Environ., 33, 3251-3268, 1999.

Charlson, R. J. and Pilat, J. H.: Climate: the influence of aerosols, J. Appl. Meteorol., 8, 1001-1002, 1969.

Charlson, R. J., Lovelock, J. E., Andreae, M. O., and Warren, S. G.: Oceanic phytoplankton, atmospheric sulphur, cloud albedo and climate, Nature, 326, 655-661, 1987.

Charlson, R. J., Seinfeld, J. H., Nenes, A., Kulmala, M., Laaksonen, A., and Facchini, M. C.: Atmospheric science - Reshaping the theory of cloud formation, Science, 292, 2025-2026, 2001.

Chen, J. P.: Theory of Deliquescence and Modified Kohler Curves, J. Atmos. Sci., 51, 3505-3516, 1994.

Chen, L. Y., Jeng, F. T., Chen, C. C., and Hsiao, T. C.: Hygroscopic behavior of atmospheric aerosol in Taipei, Atmos. Environ., 37, 2069-2075, 2003.

Chio, C.-P., Cheng, M.-T., and Wang, C.-F.: Source apportionment to PM10 in different air quality conditions for Taichung ur- 
ban and coastal areas, Taiwan, Atmos. Environ., 38, 6893-6905, 2004.

Chodes, N., Warner, J., and Gagin, A.: A determination of the condensation coefficient of water from the growth rate of small cloud droplets, J. Atmos. Sci., 31, 1351-1357, 1974.

Chuang, P. Y.: Measurement of the timescale of hygroscopic growth for atmospheric aerosols, J. Geophys. Res.-Atmos., 108, 4282, doi:10.1029/2002JD002757, 2003.

Chuang, P. Y., Charlson, R. J., and Seinfeld, J. H.: Kinetic limitations on droplet formation in clouds, Nature, 390, 594-596, 1997.

Chuang, P. Y., Nenes, A., Smith, J. N., Flagan, R. C., and Seinfeld, J. H.: Design of a CCN instrument for airborne measurement, J. Atmos. Ocean. Techn., 17, 1005-1019, 2000.

Chýlek, P. and Wong, J. G. D.: Erroneous use of the modified Köhler equation in cloud and aerosol physics application, J. Atmos. Sci., 55, 1473-1477, 1998.

Claeys, M., Graham, B., Vas, G., Wang, W., Vermeylen, R., Pashynska, V., Cafmeyer, J., Guyon, P., Andreae, M. O., Artaxo, P., and Maenhaut, W.: Formation of secondary organic aerosols through photooxidation of isoprene, Science, 303, 1173-1176, 2004.

Clarke, A., Kapustin, V., Howell, S., Moore, K., Lienert, B., Masonis, S., Anderson, T., and Covert, D.: Sea-salt size distributions from breaking waves: Implications for marine aerosol production and optical extinction measurements during SEAS, J. Atmos. Oceanic Technol., 20, 1362-1374, 2003.

Clarke, A. D., Eisele, F., Kapustin, V. N., Moore, K., Tanner, D., Mauldin, L., Litchy, M., Lienert, B., Carroll, M. A., and Albercook, G.: Nucleation in the equatorial free troposphere: Favorable environments during PEM-Tropics, J. Geophys. Res.Atmos., 104, 5735-5744, 1999.

Clegg, S. L., Brimblecombe, P., and Wexler, A. S.: Thermodynamic model of the system $\mathrm{H}+-\mathrm{NH}_{4}+-\mathrm{SO}_{4}^{2}-\mathrm{NO}_{3}-\mathrm{H}_{2} \mathrm{O}$ at tropospheric temperatures, J. Phys. Chem. A., 102, 2137-2154, 1998.

Clegg, S. L., Seinfeld, J. H., and Brimblecombe, P.: Thermodynamic modelling of aqueous aerosols containing electrolytes and dissolved organic compounds, J. Aerosol Sci., 32, 713-738, 2001.

Cocker, D. R., Whitlock, N. E., Flagan, R. C., and Seinfeld, J. H.: Hygroscopic properties of Pasadena, California aerosol, Aerosol Sci. Technol., 35, 637-647, 2001.

Coe, H. and Allan, J. D.: Analytical Techniques for Atmospheric Measurement, chap. 6, Blackwell Publishing Ltd., "Mass spectrometric and other methods for determination of the composition of aerosols", 2005.

Cohard, J.-M., Pinty, J. P., and Bedos, C.: Extending Twomey's analytical estimate of nucleated cloud droplet concentrations from CCN spectra, J. Atmos. Sci., 55, 3348-3357, 1998.

Conant, W. C., VanReken, T. M., Rissman, T. A., Varutbangkul, V., Jonsson, H. H., Nenes, A., Jimenez, J. L., Delia, A. E., Bahreini, R., Roberts, G. C., Flagan, R. C., and Seinfeld, J. H.: Aerosolcloud drop concentration closure in warm cumulus, J. Geophys. Res., 109(D13), 13 204, doi:10.1029/2003JD004324, 2004.

Collins, D. R., Flagan, R. C., and Seinfeld, J. H.: Improved inversion of scanning DMA data, Aerosol Sci. Technol., 36, 1-9, 2002.

Covert, D. S. and Heintzenberg, J.: Measurement of the Degree of Internal External Mixing of Hygroscopic Compounds and Soot in Atmospheric Aerosols, Sci. Total Environ., 36, 347-352,
1984.

Covert, D. S. and Heintzenberg, J.: Size Distributions and Chemical-Properties of Aerosol at Ny Alesund, Svalbard, Atmos. Environ., Part a-General Topics, 27, 2989-2997, 1993.

Crutzen, P. and Ramanathan, V.: The parasol effect on climate, Science, 203, 1679-1680, 2003.

Cruz, C. N. and Pandis, S. N.: The effect of organic coatings on the cloud condensation nuclei activation of inorganic atmospheric aerosol, J. Geophys. Res., 103, 13 111-13 123, 1998.

Cubison, M., Coe, H., and Gysel, M.: A modified hygroscopic tandem DMA and a data retrieval method based on optimal estimation, J. Aeros. Sci., 36, 846-865, 2005.

Davidovits, P., Worsnop, D. R., Jayne, J. T., Kolb, J. T., Winkler, P., Vrtala, A., Wagner, P. R., Kulmala, M., Lehtinen, K. E. J., Vesala, T. and Mozurkewich, M.: Mass accommodation coefficient of water vapor on liquid water, Geophys. Res. Lett., 31, L2 2111, doi:10.1029/2004GL020835, 2004.

Davidson, E. A. and Artaxo, P.: Globally significant changes in biological processes of the Amazon Basin: results of the Large-scale Biosphere-Atmosphere Experiment, Global Change Biology, 10, 519-529, 2004.

De Bock, L. A., Joos, P. E., Noone, K. J., Pockalny, R. A., and Van Grieken, R. E.: Single particle analysis of aerosols, observed in the marine boundary layer during the Monterey Area Ship Tracks Experiment (MAST), with respect to cloud droplet formation, J. Atmos. Chem., 37, 299-329, 2000.

Decesari, S., Facchini, M. C., Fuzzi, S., and Tagliavini, E.: Characterization of water-soluble organic compounds in atmospheric aerosol: A new approach, J. Geophys. Res.-Atmos., 105, 14811489, 2000.

Decesari, S., Facchini, M. C., Matta, E., Lettini, F., Mircea, M., Fuzzi, S., Tagliavini, E., and Putaud, J. P.: Chemical features and seasonal variation of fine aerosol water-soluble organic compounds in the Po Valley, Italy, Atmos. Environ., 35, 3691-3699, 2001.

Decesari, S., Facchini, M. C., Mircea, M., Cavalli, F., and Fuzzi, S.: Solubility properties of surfactants in atmospheric aerosol and cloud/fog water samples, J. Geophys. Res.-Atmos., 108, 2003.

Decesari, S., Facchini, M. C., Fuzzi, S., McFiggans, G. B., Coe, H., and Bower, K. N.: The water-soluble organic component of sizesegregated aerosol, cloud water and wet depositions from Jeju Island during ACE-Asia, Atmos. Environ., 39, 211-222, 2005.

Decesari, S., Fuzzi, S., Facchini, M. C., Maenhaut, W., Chi, X., Schkolnik, G., Falkovich, A., Rudich, Y., Claeys, M., Pashynska, V., Vas, G., Kourtchev, I., Vermeylen, R., Hoffer, A., Andreae, M. O., Tagliavini, E., Moretti, F., and Artaxo, P.: Characterization of the organic composition of aerosols from Rondônia, Brazil, during the LBA-SMOCC 2002 experiment and its representation through model compounds, Atmos. Chem. Phys., 6, 375-402, 2006, http://www.atmos-chem-phys.net/6/375/2006/.

Dick, W. D., Ziemann, P. J., Huang, P. F., and McMurry, P. H.: Optical shape fraction measurements of submicrometre laboratory and atmospheric aerosols, Measurement Science \& Technology, 9, 183-196, 1998.

Dick, W. D., Saxena, P., and McMurry, P. H.: Estimation of water uptake by organic compounds in submicron aerosols measured during the Southeastern Aerosol and Visibility Study, J. Geophys. Res.-Atmos., 105, 1471-1479, 2000. 
Drewnick, F., Jayne, J. T., Canagaratna, M., Worsnop, D. R., and Demerjian, K. L.: Measurement of ambient aerosol composition during the PMTACS-NY 2001 using an aerosol mass spectrometer. Part II: Chemically speciated mass distributions, Aerosol Sci. Technol., 38, 104-117, suppl. 1, 2004.

Drewnick, F., Hings, S. S., DeCarlo, P. F., Jayne, J. T., Gonin, M., Fuhrer, K., Weimer, S., Jimenez, J. L., Demerjian, K. L., Borrmann, S., and Worsnop, D. R.: A new time-of-flight aerosol mass spectrometer (ToF-AMS) - instrument description and first field deployment, Aerosol Sci Technol., 39, 637-658, doi:10.1080/02786820500182040 2005.

Dusek, U., Covert, D. S., Wiedensohler, A., Neususs, C., Weise, D., and Cantrell, W.: Cloud condensation nuclei spectra derived from size distributions and hygroscopic properties of the aerosol in coastal south-west Portugal during ACE-2, Tellus Series B Chemical and Physical Meteorology, 55, 35-53, 2003.

Ellison, G. B., Tuck, A. F., and Vaida, V.: Atmospheric processing of organic aerosols, J. Geophys. Res., 104, 11 633-11 641, 1999.

Ervens, B., Feingold, G., and Kreidenweis, S. M.: The influence of water-soluble organic carbon on cloud drop number concentration, J. Geophys. Res., 110, D18211, doi:10.1029/2004JD005634, 2005.

Facchini, M. C., Fuzzi, S., Zappoli, S., Andracchio, A., Gelencser, A., Kiss, G., Krivacsy, Z., Meszaros, E., Hansson, H. C., Alsberg, T., and Zebuhr, Y.: Partitioning of the organic aerosol component between fog droplets and interstitial air, J. Geophys. Res., 104, 26 821-26832, 1999a.

Facchini, M. C., Mircea, M., Fuzzi, S., and Charlson, R. J.: Cloud albedo enhancement by surface-active organic solutes in growing droplets, Nature, 401, 257-259, 1999 b.

Facchini, M. C., Decesari, S., Mircea, M., Fuzzi, S., and Loglio, G.: Surface tension of atmospheric wet aerosol and cloud/fog droplets in relation to their organic carbon content and chemical composition, Atmos. Environ., 34, 4853-4857, 2000.

Facchini, M. C., Mircea, M., Fuzzi, S., and Charlson, R. J.: Comments on "Influence of soluble surfactant properties on the activation of aerosol particles containing inorganic solute", J. Atmos. Sci., 58, 1465-1467, 2001.

Falkovich, A. H., Graber, E. R., Schkolnik, G., Rudich, Y., Maenhaut, W., and Artaxo, P.: Low molecular weight organic acids in aerosol particles from Rondonia, Brazil, during the biomassburning, transition and wet periods, Atmos. Chem. Phys., 5, 781797, 2005,

http://www.atmos-chem-phys.net/5/781/2005/.

Feichter, J., Lohmann, U., and Schult, I.: The atmospheric sulfur cycle in ECHAM-4 and its impact on the shortwave radiation, Climate Dynamics, 13, 235-246, 1997.

Feingold, G.: analysis of smoke impact on clouds in Brazilian biomass burning regions: An extension of Twomey's approach, J. Geophys. Res., 106, 22 907-22 922, 2001.

Feingold, G.: Modeling of the first indirect effect: Analysis of measurement requirements, Geophys. Res. Lett., 30, 1997, doi:10.1029/2003GL017967, 2003.

Feingold, G. and Chuang, P. Y.: Analysis of the influence of filmforming compounds on droplet growth: Implications for cloud microphysical processes and climate, J. Atmos. Sci., 59, 20062018, 2002.

Feingold, G., Jiang, H., and Harrington, J. Y.: On smoke suppression of clouds in Amazonia, Geophys. Res. Lett., 32, 2006-2018,
doi:10.1029/2004GL021369, 2005.

Ferron, G. A., Karg, E., Busch, B., and Heyder, J.: Ambient particles at an urban, semi-urban and rural site in Central Europe: hygroscopic properties, Atmos. Environ., 39, 343-352, 2005.

Fischer, K. and Grassl, H.: Absorption by airborne and deposited particles in 8-13 micrometer range, Tellus, 27, 522-528, 1975.

Flynn, M. J., Bower, K. N., Choularton, T. W., Wobrock, W., Makela, J. M., Martinsson, B., Frank, G., Hansson, H. C., Karlsson, H. and Laj, P.: Modelling cloud processing of aerosol during the ACE-2 HILLCLOUD experiment, Tellus B, 52, 779-800, 2000.

Folkers, M., Mentel, T. F., and Wahner, A.: Influence of an organic coating on the reactivity of aqueous aerosols probed by the heterogeneous hydrolysis of $\mathrm{N}_{2} \mathrm{O}_{5}$, Geophys. Res. Lett., 30, 1644 1644, 2003.

Formenti, P., Andreae, M. O., Lange, L., Roberts, G., Cafmeyer, J., Rajta, I., Maenhaut, W., Holben, B. N., Artaxo, P., and Lelieveld, J.: Saharan dust in Brazil and Suriname during the Large-Scale Biosphere-Atmosphere Experiment in Amazonia (LBA) - Cooperative LBA Regional Experiment (CLAIRE) in March 1998, J. Geophys. Res.-Atmos., 106, 14 919-14 934, 2001.

Fountoukis, C., and Nenes, A.: Continued development of a cloud droplet formation parameterization for global climate models, J. Geophys. Res., 110, D11212, doi:10.1029/2003JD004324, 2005.

Franze, T., Weller, M. G., Niessner, R., and Poeschl, U.: Protein nitration by polluted air, Environ. Sci. Technol., 39, 1673-1678, 2005.

Fredenslund, A., R. J. and Prausnitz, J.: Group-Contribution Estimation of Activity-Coefficients in Nonideal Liquid-Mixtures, Aiche Journal, 21, 1086-1099, 1975.

Freud, E., Rosenfeld, D., Andreae, M. O., Costa, A., and Artaxo, P.: Robust relations between $\mathrm{CCN}$ and vertical evolution of cloud drop size distributions in deep convective clouds, Atmos. Chem. Phys. Discuss., 5, 10 155-10 195, 2005.

Fuzzi, S., Decesari, S., Facchini, M. C., Matta, E., Mircea, M., and Tagliavini, E.: A simplified model of the water soluble organic component of atmospheric aerosols, Geophys. Res. Lett., 28, 4079-4082, 2001.

Gaman, A. I., Kulmala, M., Vehkamaki, H., Napari, I., Mircea, M., Facchini, M. C., and Laaksonen, A.: Binary homogeneous nucleation in water-succinic acid and water-glutaric acid systems, J. Chem. Phys., 120, 282-291, 2004.

Gao, S., Ng, N. L., Keywood, M., Varutbangkul, V., Bahreini, R., Nenes, A., He, J. W., Yoo, K. Y., Beauchamp, J. L., Hodyss, R. P., Flagan, R. C., and Seinfeld, J. H.: Particle phase acidity and oligomer formation in secondary organic aerosol, Environ. Sci. Technol., 38, 6582-6589, 2004.

Gerber, H. E., Hoppel, W. A., and Wojciechowski, T. .: Experimental verification of the theoretical relationship between size and critical supersaturation of salt nuclei, J. Atmos. Sci., 34, 18361841, 1977.

Ghan, S. J., Leung, L. R., Easter, R. C., and Abdul-Razzak, H.: Prediction of cloud droplet number in a general circulation model, J. Geophys. Res., 102, 777-794, 1997.

Ghan, S. J., Guzman, G., and Abdul-Razzak, H.: Competition between sea salt and sulfate particles as cloud condensation nuclei, J. Atmos. Sci., 55, 3340-3347, 1998.

Ghan, S. J., Easter, R. C., Chapman, E., Abdul-Razzak, H., Zhang, Y., Leung, L. R., Laulainen, N., Saylor, R., and Zaveri, R.: A 
physically-based estimate of radiative forcing by anthropogenic sulphate aerosols, J. Geophys. Res., 106, 5279-5293, 2001.

Giebl, H., Berner, A., Reischl, G., Puxbaum, H., Kasper-Giebl, A., and Hitzenberger, R.: CCN activation of oxalic and malonic acid test aerosols with the University of Vienna cloud condensation nuclei counter, J. Aerosol Sci., 33, 1623-1634, 2002.

Gill, P. S., Graedel, T. E., and Weschler, C. J.: Organic Films on Atmospheric Aerosol-Particles, Fog Droplets, Cloud Droplets, Raindrops, and Snowflakes, Rev. Geophys., 21, 903-920, 1983.

Gilman, J. B., Eliason, T. L., Fast, A., and Vaida, V.: Selectivity and stability of organic films at the air-aqueous interface, Journal of Colloid and Interface Science, 280, 234-243, 2004.

Gong, S. L. and Barrie, L. A.: Simulating the impact of sea salt on global nss sulphate aerosols, J. Geophys. Res.-Atmos., 108, 4516, doi:10.1029/2002JD003181, 2003.

Gong, S. L., Barrie, L. A., and Blanchet, J. P.: Modeling sea-salt aerosols in the atmosphere .1. Model development, J. Geophys. Res.-Atmos., 102, 3805-3818, 1997.

Gorbunov, B. and Hamilton, R.: Water nucleation on aerosol particles containing both soluble and insoluble substances, J. Aeros. Sci., 28, 239-248, 1997.

Gorbunov, B., Hamilton, R., Clegg, N., and Toumi, R.: Water nucleation on aerosol particles containing both organic and soluble inorganic substances, Atmos. Res., 48, 271-283, 1998.

Graedel, T. E. and Weschler, C. J.: Chemistry within Aqueous Atmospheric Aerosols and Raindrops, Rev. Geophys., 19, 505-539, 1981.

Graham, B., Mayol-Bracero, O. L., Guyon, P., Roberts, G. C., Decesari, S., Facchini, M. C., Artaxo, P., Maenhaut, W., Koll, P., and Andreae, M. O.: Water-soluble organic compounds in biomass burning aerosols over Amazonia - 1. Characterization by NMR and GC-MS, J. Geophys. Res.-Atmos., 107, 8047, doi:10.1029/2001JD000336, 2002.

Graham, B., Guyon, P., Maenhaut, W., Taylor, P. E., Ebert, M., Matthias-Maser, S., Mayol-Bracero, O. L., Godoi, R. H. M., Artaxo, P., Meixner, F. X., Moura, M. A. L., Rocha, C., Van Grieken, R., Glovsky, M. M., Flagan, R. C., and Andreae, M. O.: Composition and diurnal variability of the natural Amazonian aerosol, J. Geophys. Res.-Atmos., 108, 4765-4782, 2003a.

Graham, B., Guyon, P., Taylor, P. E., Artaxo, P., Maenhaut, W., Glovsky, M. M., Flagan, R. C., and Andreae, M. O.: Organic compounds present in the natural Amazonian aerosol: Characterization by gas chromatography-mass spectrometry, J. Geophys. Res.-Atmos., 108, 4766, doi:10.1029/2003JD003990, 2003 b.

Guibert, S., Snider, J. R., and Brenguier, J. L.: Aerosol activation in marine stratocumulus clouds: 1 . Measurement validation for a closure study, J. Geophys. Res.-Atmos., 108, 8628, doi:10.1029/2002JD002678, 2003.

Gysel, M., Weingartner, E., Nyeki, S., Paulsen, D., Baltensperger, U., Galambos, I., and Kiss, G.: Hygroscopic properties of water-soluble matter and humic-like organics in atmospheric fine aerosol, Atmos. Chem. Phys., 4, 35-50, 2004, http://www.atmos-chem-phys.net/4/35/2004/.

Hallberg, A., Wobrock, W., Flossmann, A. I., Bower, K. N., Noone, K. J., Wiedensohler, A., Hansson, H. C., Wendisch, M., Berner, A., Kruisz, C., Laj, P., Facchini, M. C., Fuzzi, S., and Arends, B. G.: Microphysics of clouds: Model vs measurements, Atmos. Environ., 31, 2453-2462, 1997.
Hämeri, K., Vakeva, M., Aalto, P. P., Kulmala, M., Swietlicki, E., Zhou, J., Seidl, W., Becker, E., and O'Dowd, C. D.: Hygroscopic and $\mathrm{CCN}$ properties of aerosol particles in boreal forests, Tellus Series B - Chemical and Physical Meteorology, 53, 359-379, 2001.

Hamilton, J. F., Webb, P. J., Lewis, A. C., Hopkins, J. R., Smith, S., and Davy, P.: Partially oxidised organic components in urban aerosol using GCXGC-TOF/MS, Atmos. Chem. Phys., 4, 12791290, 2004, http://www.atmos-chem-phys.net/4/1279/2004/.

Hänel, G.: Academic Press, New York, the Properties of Atmospheric Aerosol Particles as Functions of the Relative Humidity at Themodynamic Equilibrium with the Surrounding Moist Air, Adv. Geophys., 19, 73-188, 1976.

Hansen, J., Sato, M., Lacis, A., and Ruedy, R.: The missing climate forcing, Philos. T. Roy. Soc., 352, 231-240, 1997.

Haywood, J. M. and Boucher, O.: Estimates of the direct and indirect radiative forcing due to tropospheric aerosols: A review, Rev. Geophys., 38, 513-543, 2000.

Hegg, D. A.: Impact of gas-phase HNO3 and NH3 on microphysical processes in atmospheric clouds, Geophys. Res. Lett., 27, 2201-2204, 2000

Heintzenberg, J.: Fine particles in the global troposphere, A review, Tellus Series B - Chemical and Physical Meteorology, 41, 149160, 1989.

Heintzenberg, J. and Covert, D. S.: Chemically resolved submicrometric size distributions and external mixing of the Arctic haze aerosols, Tellus B, 39, 374-382, 1987.

Heintzenberg, J., Covert, D. S., and Van Dingenen, R.: Size distribution and chemical composition of marine aerosols: a compilation and review, Tellus B, 52, 1104-1122, 2000.

Henning, S., Weingartner, E., Schmidt, S., Wendisch, M., Gaggeler, H. W., and Baltensperger, U.: Size-dependent aerosol activation at the high-alpine site Jungfraujoch (3580 $\mathrm{m}$ a.s.1.), Tellus, 54B, 82-95, 2002.

Henning, S., Rosenorn, T., D’ Anna, B., Gola, A. A., Svenningsson, B., and Bilde, M.: Cloud droplet activation and surface tension of mixtures of slightly soluble organics and inorganic salt, Atmos. Chem. Phys., 5, 575-582, 2005,

http://www.atmos-chem-phys.net/5/575/2005/.

Hitzenberger, R., Berner, A., Kasper-Giebl, A., Loflund, M., and Puxbaum, H.: Surface tension of Rax cloud water and its relation to the concentration of organic material, J. Geophys. Res., 107, 4752, doi:10.1029/2002JD002506, 2002.

Hoppel, W. A., Frick, G. M., and Fitzgerald, J. W.: Deducing droplet concentration and supersaturation in marine boundary layer clouds from surface aerosol measurements, J. Geophys. Res., 101, 26 553-26565, 1996.

Howell, W. E.: The growth of cloud drops in uniformly cooled air, J. Meteorol., 6, 134-149, 1949.

Hudson, J. G.: An instantaneous CCN spectrometer, J. Atmos. Oceanogr. Techn., 6, 1055-1065, 1989.

Ion, A. C., Vermeylen, R., Kourtchev, I., Cafmeyer, J., Chi, X., Gelencsér, A., Maenhaut, W., and Claeys, M.: Polar organic compounds in rural PM2.5 aerosols from K-puszta, Hungary, during a 2003 summer field campaign: sources and diurnal variations, Atmos. Chem. Phys., 5, 1805-1814, 2005, http://www.atmos-chem-phys.net/5/1805/2005/.

Jacobson, M. C., Hansson, H. C., Noone, K. J., and Charlson, R. J.: 
Organic atmospheric aerosols: Review and state of the science, Rev. Geophys., 38, 267-294, 2000.

Jaenicke, R.: Abundance of cellular material and proteins in the atmosphere, Science, 308(5718), 73-73, 2005.

Jayne, J. T., Leard, D. C., Zhang, X. F., Davidovits, P., Smith, K. A., Kolb, C. E., and Worsnop, D. R.: Development of an aerosol mass spectrometer for size and composition analysis of submicron particles, Aerosol Sci. Technol., 33, 49-70, 2000.

Ji, Q., Shaw, G. E., and Cantrell, W.: A new instrument for measuring cloud condensation nuclei: Cloud condensation nucleus "remover", J. Geophys. Res.-Atmos., 103, 28 013-28 019, 1998.

Jimenez, J. L., Jayne, J. T., Shi, Q., Kolb, C. E., Worsnop, D. R., Yourshaw, I., Seinfeld, J. H., Flagan, R. C., and Zhang, X, Smith, K. A., Morris, J, and Davidovits, P.: Ambient Aerosol Sampling with an Aerosol Mass Spectrometer., J. Geophys. Res., 108, 8425, doi:10.1029/2001JD001213, 2003.

Johnson, B. T., Shine, K. P., and Forster, P. M.: The semi-direct aerosol effect: Impact of absorbing aerosols on marine stratocumulus, Quart. J. Roy. Meteorol. Soc., 130, 1407-1422, 2004.

Johnson, G. R., Ristovski, Z. D., D’Anna, B., and Morawska, L.: The hygroscopic behavior of partially volatilized coastal marine aerosols using the VH-TDMA technique, J. Geophys. Res.-A., 110, D20203, doi:10.1029/2004JD005657, 2005.

Jones, A., Roberts, D. L., and Slingo, A.: A Climate Model Study of Indirect Radiative Forcing by Anthropogenic Sulfate Aerosols, Nature, 370, 450-453, 1994.

Jones, A., Roberts, D. L., Woodage, M. J., and Johnson, C. E.: Indirect sulphate aerosol forcing in a climate model with an interactive sulphur cycle, J. Geophys. Res.-Atmos., 106, 20 293-20310, 2001.

Kalberer, M., Paulsen, D., Sax, M., Steinbacher, M., Dommen, J., Prevot, A. S. H., Fisseha, R., Weingartner, E., Frankevich, V., Zenobi, R., and Baltensperger, U.: Identification of polymers as major components of atmospheric organic aerosols, Science, 303, 1659-1662, 2004.

Kanakidou, M., Seinfeld, J. H., Pandis, S. N., Barnes, I., Dentener, F. J., Facchini, M. C., Van Dingenen, R., Ervens, B., Nenes, A., Nielsen, C. J., Swietlicki, E., Putaud, J. P., Balkanski, Y., Fuzzi, S., Horth, J., Moortgat, G. K., Winterhalter, R., Myhre, C. E. L., Tsigaridis, K., Vignati, E., Stephanou, E. G., and Wilson, J.: Organic aerosol and global climate modelling: a review, Atmos. Chem. Phys., 5, 1053-1123, 2005,

http://www.atmos-chem-phys.net/5/1053/2005/.

Katrib, Y., Martin, S. T., Hung, H. M., Rudich, Y., Zhang, H. Z., Slowik, J. G., Davidovits, P., Jayne, J. T. and Worsnop, D.R.: Products and mechanisms of ozone reactions with oleic acid for aerosol particles having core-shell morphologies, J. Phys. Chem. A, 108, 6686-6695, 2004.

Katz, U. and Kocmond, W. C.: Investigation of SizeSupersaturation Relationship of Soluble Condensation Nuclei, J. Atmos. Sci., 30, 160-165, 1973.

Kinne, S., Lohmann, U., Feichter, J., Schulz, M., Timmreck, C., Ghan, S., Easter, R., Chin, M., Ginoux, P., Takemura, T., Tegen, I., Koch, D., Herzog, M., Penner, J., Pitari, G., Holben, B., Eck, T., Smirnov, A., Dubovik, O., Slutsker, I., Tanre, D., Torres, O., Mishchenko, M., Geogdzhayev, I., Chu, D. A., and Kaufman, Y.: Monthly averages of aerosol properties: A global comparison among models, satellite data, and AERONET ground data, J. Geophys. Res.-Atmos., 108, 4634, doi:10.1029/2001JD001253,
2003.

Kiss, G. and Hansson, H.-C.: Application of osmolality for the determination of water activity and the modelling of cloud formation, Atmos. Chem. Phys. Discuss., 4, 7667-7689, 2004,

http://www.atmos-chem-phys-discuss.net/4/7667/2004/.

Kiss, G., Varga, B., Gelencsér, A., Krivacsy, Z., Molnar, A., Alsberg, T., Persson, L., Hansson, H. C., and Facchini, M. C.: Characterisation of polar organic compounds in fog water, Atmos. Environ., 35, 2193-2200, 2001.

Kiss, G., Varga, B., Galambos, I., and Ganszky, I.: Characterization of water-soluble organic matter isolated from atmospheric fine aerosol, J. Geophys. Res., 107, 8339, doi:10.1029/2001JD000603, 2002.

Kiss, G., Tombácz, E., and Hansson, H.-C.: Surface tension effects of humic-like substances in the aqueous extract of tropospheric fine aerosol, J. Atmos. Chem., 50, 279-294, doi:10.1007/s10874-005-5079-5, 2005.

Kokkola, H., Romakkaniemi, S., and Laaksonen, A.: Kohler theory for a polydisperse droplet population in the presence of a soluble trace gas, and an application to stratospheric STS droplet growth, Atmos. Chem. Phys., 3, 2139-2146, 2003,

http://www.atmos-chem-phys.net/3/2139/2003/.

Komppula, M., Lihavainen, H., Kerminen, V. M., Kulmala, M., and Viisanen, Y.: Measurements of cloud droplet activation of aerosol particles at a clean subarctic background site, J. Geophys. Res.-Atmos., 110, D06 204, doi:10.1029/2004JD005200, 2005.

Koren, I., Kaufman, Y. J., Remer, L. A., and Martins, J. V.: Measurement of the effect of Amazon smoke on inhibition of cloud formation, Science, 303, 1342-1345, 2004.

Kreidenweis, S. M., McInnes, L. M., and Brechtel, F. J.: Observations of aerosol volatility and elemental composition at Macquarie Island during the First Aerosol Characterization Experiment (ACE 1), J. Geophys. Res.-Atmos., 103, 16 511-16524, 1998.

Kristjansson, J. E.: Studies of the aerosol indirect effect from sulfate and black carbon aerosols, J. Geophys. Res.-Atmos., 107, 4246, doi:10.1029/2001JD000887, 2002.

Kulmala, M., Laaksonen, A., Korhonen, P., Vesala, T., Ahonen, T., and Barrett, J. C.: The Effect of Atmospheric Nitric-Acid Vapor on Cloud Condensation Nucleus Activation, J. Geophys. Res.Atmos., 98, 22 949-22 958, 1993.

Kulmala, M., Korhonen, P., Vesala, T., Hansson, H. C., Noone, K., and Svenningsson, B.: The effect of hygroscopicity on cloud droplet formation, Tellus Series B - Chemical and Physical Meteorology, 48, 347-360, 1996.

Kulmala, M., Laaksonen, A., Charlson, R. J., and Korhonen, P.: Clouds without supersaturation, Nature, 388, 336-337, 1997.

Kulmala, M., Toivonen, A., Mattila, T., and Korhonen, P.: Variations of cloud droplet concentrations and the optical properties of clouds due to changing hygroscopicity: A model study, J. Geophys. Res.-Atmos., 103, 16 183-16 195, 1998.

Kuznetsova, M., Lee, C., and Aller, J.: Characterization of the proteinaceous matter in marine aerosols, Marine Chemistry, 96(34), 359-377, 2005.

Laaksonen, A.: The Composition Size Dependence of Aerosols Created by Dispersion of Surfactant Solutions, Journal of Colloid and Interface Science, 159, 517-519, 1993.

Laaksonen, A., Hienola, J., Kulmala, M., and Arnold, F.: Supercooled cirrus cloud formation modified by nitric acid pollution 
of the upper troposphere, Geophys. Res. Lett., 24, 3009-3012, 1997.

Laaksonen, A., Korhonen, P., Kulmala, M., and Charlson, R. J.: Modification of the Köhler equation to include soluble trace gases and slightly soluble substances, J. Atmos. Sci., 55, 853862, 1998.

Laaksonen, A., Vesala, T., Kulmala, M., Winkler, P. M., and Wagner, P. E.: Commentary on cloud modelling and the mass accommodation coefficient of water, Atmos. Chem. Phys., 5, 461-464, 2005 , http://www.atmos-chem-phys.net/5/461/2005/.

Laktionov, A. G.: A constant-temperature method of determining the concentrations of cloud condensation nuclei, Atmos. Oceanic Phys., 8, 672-677, 1972.

Lala, G. G. and Jiusto, J. E.: Automatic Light-Scattering Ccn Counter, J. Appl. Meteorol., 16, 413-418, 1977.

Lance, S., Nenes, A., and Rissman, T. A.: Chemical and dynamical effects on cloud droplet number: Implications for estimates of the aerosol indirect effect, J. Geophys. Res.-Atmos., 109, D22 208, doi:10.1029/2004JD004596, 2004.

Langner, J. and Rodhe, H.: A global three-dimensional model of the tropospheric sulphur cycle, J. Atmos. Chem., 13, 225-263, 1991.

Leaitch, R. and Megaw, W. J.: The Diffusion Tube - a Cloud Condensation Nucleus Counter for Use Below 0.3-Percent Supersaturation, J. Aerosol Sci., 13, 297-319, 1982.

Leaitch, W. R., Banic, C. M., Isaac, G. A., Couture, M. D., Liu, P. S. K., Gultepe, I., and Li, S.-M.: Physical and chemical observations in marine stratus during the 1993 North Atlantic Regional Experiment: Factors controlling cloud droplet number concentrations, J. Geophys. Res., 101, 29 123-29 135, 1996.

Leaitch, W. R., Bottenheim, J. W., Biesenthal, T. A., Li, S.-M., Liu, P., Asalain, K., Dryfhouft-Clark, H., and Hopper, F.: A case for gas-to-particle conversion in an eastern Canadian forest, J. Geophys. Res., 104, 8095-8111, 1999.

Lee, S. H., Murphy, D. M., Thomson, D. S., and Middlebrook, A. M.: Chemical components of single particles measured with Particle Analysis by Laser Mass Spectrometry (PALMS) during the Atlanta SuperSite Project: Focus on organic/sulfate, lead, soot, and mineral particles Source, J. Geophys. Res., 107(D1), 4003, doi:10.1029/2000JD000011, 2002.

Li, Z. D., Williams, A. L., and Rood, M. J.: Influence of soluble surfactant properties on the activation of aerosol particles containing inorganic solute, J. Atmos. Sci., 55, 1859-1866, 1998.

Liou, K. N. and Ou, S. C.: The role of cloud microphysical processes in climate - an assessment from a one-dimensional perspective, J. Geophys. Res., 94, 8599-8607, 1989.

Liousse, C., Penner, E., Chuang, C., Walton, J., Eddleman, H., and Cachier, H.: A global three-dimensional model study of carbonaceous aerosols, J. Geophys. Res., 101, 19411-19432, 1996.

Liu, B. Y. H. and Pui, D. Y. H.: Submicron Aerosol Standard and Primary, Absolute Calibration of Condensation Nuclei Counter, Journal of Colloid and Interface Science, 47, 155-171, 1974.

Liu, B. Y. H., Pui, D. Y. H., Whitby, K. T., Kittelson, D. B., Kousaka, Y., and McKenzie, R. L.: Aerosol Mobility Chromatograph - New Detector for Sulfuric-Acid Aerosols, Atmos. Environ., 12, 99-104, 1978.

Liu, X., Hegg, D. A. and Stoelinga, M. T.: Numerical simulation of new particle formation over the northwest Atlantic using the
MM5 mesoscale model coupled with sulfur chemistry, J. Geophys. Res., 106, 9697-9715, 2001.

Lohmann, U. and Feichter, J.: Global indirect aerosol effects: a review, Atmos. Chem. Phys., 5, 715-737, 2005, http://www.atmos-chem-phys.net/5/715/2005/.

Lohmann, U., Feichter, J., Chuang, C. C., and Penner, J. E.: Prediction of the number of cloud droplets in the ECHAM GCM, J. Geophys. Res.-Atmos., 104, 9169-9198, 1999.

Lohmann, U., Feichter, J., Penner, J., and Leaitch, R.: Indirect effect of sulfate and carbonaceous aerosols: A mechanistic treatment, J. Geophys. Res.-Atmos., 105, 12 193-12 206, 2000.

Lohmann, U., Broekhuizen, K., Leaitch, R., Shantz, N., and Abbatt, J.: How efficient is cloud droplet formation of organic aerosols?, Geophys. Res. Lett., 31, L05 108, doi:10.1029/2003GL018999, 2004.

Mace, K. A., Duce, R. A., and Tindale, N. W.: Organic nitrogen in rain and aerosol at Cape Grim, Tasmania, Australia, J. Geophys. Res., 108(D11), 4338, doi:10.1029/2002JD003051, 2003a.

Mace, K. A., Kubilay, N., and Duce, R. A.: Organic nitrogen in rain and aerosol in the eastern Mediterranean atmosphere: An association with atmospheric dust, J. Geophys. Res., 108(D10), 4320, doi:10.1029/2002JD002997, 2003b.

Mace, K. A., Artaxo, P., and Duce, R. A.: Water-soluble organic nitrogen in Amazon Basin aerosols during the dry (biomass burning) and wet seasons, J. Geophys. Res., 108(D16), 4512, doi:10.1029/2003JD003557, 2003c.

MacIntyr, F.: Top millimeter of ocean, Scientific American, 230, 5, 62, 1974.

Marcolli, C., Luo, B. P., and Peter, T.: Mixing of the organic aerosol fractions: Liquids as the thermodynamically stable phases, J. Phys. Chem.-Atmos., 108, 2216-2224, 2004.

Maria, S. F., Russell, L. M., Turpin, B. J., and Porcja, R. J.: FTIR measurements of functional groups and organic mass in aerosol samples over the Caribbean, Atmos. Environ., 36, 5185-5196, 2002.

Maria, S. F., Russell, L. M., Turpin, B. J., Porcja, R. J., Campos, T. L., Weber, R. J., and Huebert, B. J.: Source signatures of carbon monoxide and organic functional groups in Asian $\mathrm{Pa}$ cific Regional Aerosol Characterization Experiment (ACE-Asia) submicron aerosol types, J. Geophys. Res.-Atmos., 108, 8637, doi:10.1029/2003JD003703, 2003.

Maria, S. F., Russell, L. M., Gilles, M. K., and Myneni, S. C. B.: Organic aerosol growth mechanisms and their climate-forcing implications, Science, 306, 1921-1924, 2004.

Martinsson, B. G., Frank, G., Cederfelt, S. I., Swietlicki, E., Berg, O. H., Zhou, J. C., Bower, K. N., Bradbury, C., Birmili, W., Stratmann, F., Wendisch, M., Wiedensohler, A., and Yuskiewicz, B. A.: Source signatures of carbon monoxide and organic functional groups in Asian Pacific Regional Aerosol Characterization Experiment (ACE-Asia) submicron aerosol types, Atmos. Res., 50, 289-315, 1999.

Mason, B. J.: The role of sea-salt particles as cloud condensation nuclei over the remote oceans, Quart. J. Roy. Meteorol. Soc., 127, 2023-2032, part B, 2001.

Maßling, A., Wiedensohler, A., Busch, B., Neususs, C., Quinn, P., Bates, T., and Covert, D.: Hygroscopic properties of different aerosol types over the Atlantic and Indian Oceans, Atmos. Chem. Phys., 3, 1377-1397, 2003,

http://www.atmos-chem-phys.net/3/1377/2003/. 
Matsumoto, K. and Uematsu, M.: Free amino acids in marine aerosols over the western North Pacific Ocean, Atmos. Environ., 39(11), 2163-2170, 2005.

Matta, E., Facchini, M. C., Decesari, S., Mircea, M., Cavalli, F., Fuzzi, S., Putaud, J. P., and Dell'Acqua, A.: Mass closure on the chemical species in size-segregated atmospheric aerosol collected in an urban area of the Po Valley, Italy, Atmos. Chem. Phys., 3, 623-637, 2003, http://www.atmos-chem-phys.net/3/623/2003/.

Mayol-Bracero, O. L., Guyon, P., Graham, B., Roberts, G., Andreae, M. O., Decesari, S., Facchini, M. C., Fuzzi, S., and Artaxo, P.: Water-soluble organic compounds in biomass burning aerosols over Amazonia, 2. Apportionment of the chemical composition and importance of the polyacidic fraction, J. Geophys. Res.-Atmos., 107, 8091, doi:10.1029/2001JD000522, 2002.

McCormick, R. and Ludwig, J. H.: Climate Modification by Atmospheric Aerosols, Science, 156, 1358-1359, 1967.

McFiggans, G., Alfarra, M. R., Allan, J. D., Bower, K. N., Coe, H., Cubison, M., Topping, D. O., Williams, P. I., Decesari, S., Facchini, M. C., and Fuzzi, S.: Simplification of the representation of the organic component of atmospheric particulates, Faraday Discuss., 130, 1-22, doi:10.1039/b419435g, 2005.

McMurry, P. H. and Stolzenburg, M. R.: On the Sensitivity of Particle-Size to Relative-Humidity for Los-Angeles Aerosols, Atmos. Environ., 23, 497-507, 1989.

Medina, J. and Nenes, A.: Effects of film-forming compounds on the growth of giant cloud condensation nuclei: Implications for cloud microphysics and the aerosol indirect effect, J. Geophys. Res., 109, D20 207, doi:10.1029/2004JD004666, 2004.

Menon, S., Del Genio, A. D., Koch, D., and Tselioudis, G.: GCM Simulations of the aerosol indirect effect: Sensitivity to cloud parameterization and aerosol burden, J. Atmos. Sci., 59, 692713, 2002.

Meskhidze, N., Nenes, A., Conant, W. C. and Seinfeld, J. H.: Evaluation of a new cloud droplet activation parameterization with in situ data from CRYSTAL-FACE and CSTRIPE, J. Geophys. Res., 110, D16 202, doi:10.1029/2004JD00573, 2005.

Middlebrook, A. M., Thomson, D. S., and Murphy, D. M.: On the purity of laboratory generated sulfuric acid droplets and ambient particles studied by laser mass spectrometry, Aerosol Sci. Technol., 27, 293-307, 1997.

Middlebrook, A. M., Murphy, D. M., and Thomson, D. S.: Observations of organic material in individual marine particles at Cape Grim during the First Aerosol Characterization Experiment (ACE 1), J. Geophys. Res.-Atmos., 103, 16 475-16483, 1998.

Miguel, A. G., Cass, G. R., Glovsky, M. M., and Weiss, J.: Allergens in paved road dust and airborne particles, Environ. Sci. Technol., 33(23), 4159-4168, 1999.

Mikhailov, E., Vlasenko, S., Niessner, R., and Poschl, U.: Interaction of aerosol particles composed of protein and salts with water vapor: hygroscopic growth and microstructural rearrangement, Atmos. Chem. Phys., 4, 323-350, 2004, http://www.atmos-chem-phys.net/4/323/2004/.

Ming, Y. and Russell, L. M.: Thermodynamic equilibrium of organic-electrolyte mixtures in aerosol particles, Aiche Journal, 48, 1331-1348, 2002.

Ming, Y. and Russell, L. M.: Organic aerosol effects on fog droplet spectra, J. Geophys. Res.-Atmos., 109, D10 206, doi:10.1029/2003JD004427, 2004.
Mircea, M., Facchini, M. C., Decesari, S., Fuzzi, S., and Charlson, R. J.: The influence of the organic aerosol component on CCN supersaturation spectra for different aerosol types, Tellus Series B - Chemical and Physical Meteorology, 54, 74-81, 2002.

Mochida, M., Kitamori, Y., Kawamura, K., Nojiri, Y., and Suzuki, K.: Fatty acids in the marine atmosphere: Factors governing their concentrations and evaluation of organic films on sea-salt particles, J. Geophys. Res.-Atmos., 107, 4325, doi:10.1029/2001JD001278, 2002.

Moise, T. and Rudich, Y.: Reactive uptake of ozone by aerosolassociated unsaturated fatty acids: Kinetics, mechanism, and products, J. Phys. Chem. A, 106(27), 6469-6476, 2002.

Mordy, W. A.: Computations of the growth by condensation of a population of cloud droplets, Tellus, 11, 16-44, 1959.

Mozurkewich, M.: Aerosol growth and the condensation coefficient for water - a review, Aerosol Sci. Technol., 5, 223-236, 1986.

Murphy, D. M.: Something in the air, Science, 307, 1888-1890, 2005.

Murphy, D. M., Anderson, J. R., Quinn, P. K., McInnes, L. M., Brechtel, F. J., Kreidenweis, S. M., Middlebrook, A. M., Posfai, M., Thomson, D. S., and Buseck, P. R.: Influence of sea-salt on aerosol radiative properties in the Southern Ocean marine boundary layer, Nature, 392, 62-65, 1998.

Nenes, A., Chuang, P. Y., Flagan, R. C., and Seinfeld, J. H.: A theoretical analysis of cloud condensation nucleus (CCN) instruments, J. Geophys. Res.-Atmos., 106, 3449-3474, 2001a.

Nenes, A., Ghan, S., Abdul-Razzak, H., Chuang, P. Y., and Seinfeld, J. H.: Kinetic limitations on cloud droplet formation and impact on cloud albedo, Tellus Series B - Chemical and Physical Meteorology, 53, 133-149, 2001 b.

Nenes, A., Charlson, R. J., Facchini, M. C., Kulmala, M., Laaksonen, A., and Seinfeld, J. H.: Can chemical effects on cloud droplet number rival the first indirect effect?, Geophys. Res. Lett., 29, 1848, doi:10.1029/2002GL015295, 2002.

Nenes, A. and Seinfeld, J. H.: Parameterization of cloud droplet formation in global climate models, J. Geophys. Res., 108, 4415, doi:10.1029/2002JD002911, 2003.

Nessler, R., Bukowiecki, N., Henning, S., Weingartner, E., Calpini, B., and Baltensperger, U.: Simultaneous dry and ambient measurements of aerosol size distributions at the Jungfraujoch, Tellus, 55B, 808-819, 2003.

Noble, C. A. and Prather, K. A.: Real-time single particle mass spectrometry: A historical review of a quarter century of the chemical analysis of aerosols, Mass Spectrometry Reviews, 19, 248-274, 2000.

O'Dowd, C. D., Smith, M. H., Consterdine, I. E., and Lowe, J. A.: Marine aerosol, sea-salt, and the marine sulphur cycle: A short review, Atmos. Environ., 31, 73-80, 1997.

O'Dowd, C. D., Lowe, J. A., Smith, M. H., and Kaye, A. D.: The relative importance of non-sea-salt sulphate and sea-salt aerosol to the marine cloud condensation nuclei population: An improved multi-component aerosol-cloud droplet parametrization, Quart. J. Roy. Meteorol. Soc., part B, 125, 1295-1313, 1999.

O’Dowd, C. D., Facchini, M. C., Cavalli, F., Ceburnis, D., Mircea, M., Decesari, S., Fuzzi, S., Yoon, Y. J., and Putaud, J. P.: Biogenically driven organic contribution to marine aerosol, Nature, 431, 676-680, 2004.

Oliveira, D. J. and Vali, G.: Calibration of a photoelectric cloud condensation nucleus counter, Atmos. Res., 38, 237-248, 1995. 
Otto, P., Georgii, H. W., and Bingemer, H.: A new three-stage continuous flow CCN-counter, Atmos. Res., 61, 299-310, 2002.

Pagels, J., Strand, M., Rissler, J., Szpila, A., Gudmundsson, A., Bohgard, M., Lillieblad, L., Sanati, M., and Swietlicki, E.: Characteristics of aerosol particles formed during grate combustion of moist forest residue, J. Aerosol Sci., 34, 1043-1059, 2003

Pakkanen, T. A., Loukkola, K., Korhonen, C. H., Aurela, M., Makela, T., Hillamo, R. E., Aarnio, P., Koskentalo, T., Kousa, A., and Maenhaut, W.: Sources and chemical composition of atmospheric fine and coarse particles in the Helsinki area, Atmos. Environ., 35, 5381-5391, 2001.

Pashynska, V., Vermeylen, R., Vas, G., Maenhaut, W., and Claeys, M.: Development of a gas chromatographic/ion trap mass spectrometric method for the determination of levoglucosan and saccharidic compounds in atmospheric aerosols. Application to urban aerosols, J. Mass Spectr., 37, 1249-1257, 2002.

Patris, N., Mihalopoulos, N., Baboukas, E. D., and Jouzel, J.: Isotopic composition of sulfur in size-resolved marine aerosols above the Atlantic Ocean, J. Geophys. Res., 105, 14 449-14457, 2000.

Peng, C., Chan, M. N., and Chan, C.: The hygroscopic properties of dicarboxylic and multifunctional acids: Measurements and UNIFAC predictions, Environ. Sci. Technol., 35, 4495-4501, 2001.

Peng, Y., Lohmann, U., and Leaitch, R.: Importance of vertical velocity variations in the cloud droplet nucleation process of marine stratus clouds, J. Geophys. Res., 110, D21 213, doi:10.1029/2004JD004922, 2005.

Petzold, A., Gysel, M., Vancassel, X., Hitzenberger, R., Puxbaum, H., Vrochticky, S., Weingartner, E., Baltensperger, U., and Mirabel, P.: On the effects of organic matter and sulphurcontaining compounds on the $\mathrm{CCN}$ activation of combustion particles, Atmos. Chem. Phys., 5, 3187-3203, 2005, http://www.atmos-chem-phys.net/5/3187/2005/.

Phinney, L. A., Lohmann, U., and Leaitch, W. R.: Limitations of using an equilibrium approximation in an aerosol activation parameterization, J. Geophys. Res.-Atmos., 108, 4371, doi:10.1029/2002JD002391, 2003.

Pitchford, M. L. and McMurry, P. H.: Relationship between Measured Water-Vapor Growth and Chemistry of Atmospheric Aerosol for Grand-Canyon, Arizona, in Winter 1990, Atmos. Environ., 28, 827-839, 1994.

Poeschl, U.: Atmospheric aerosols: composition, transformation, climate and health effects, Angewandte Chemie International Edition, doi:10.1002/ange.200501122, 2005.

Procopio, A. S., Remer, L. A., Artaxo, P., Kaufman, Y. J., and Holben, B. N.: Modeled spectral optical properties for smoke aerosols in Amazonia, Geophys. Res. Lett., 30, 2265, doi:10.1029/2003GL018063, 2003.

Procopio, A. S., Artaxo, P., Kaufman, Y. J., Remer, L. A., Schafer, J. S., and Holben, B. N.: Multiyear analysis of amazonian biomass burning smoke radiative forcing of climate, Geophys. Res. Lett., 31, L03 108, doi:10.1029/2003GL018646, 2004.

Pruppacher, H. and Klett, J.: Microphysics of clouds and precipitation, Atmospheric and oceanographic sciences library; v. 18, Kluwer Academic Publishers, Dordrecht; London, includes bibliographical references and index Previous ed.: 1978 "With an introduction to cloud chemistry and cloud electricity.", 1997.

Putaud, J. P., Van Dingenen, R., Mangoni, M., Virkkula, A., Raes, F., Maring, H., Prospero, J. M., Swietlicki, E., Berg, O. H.,
Hillamo, R., and Makela, T.: Chemical mass closure and assessment of the origin of the submicron aerosol in the marine boundary layer and the free troposphere at Tenerife during ACE-2, Tellus Series B - Chemical and Physical Meteorology, 52, 141-168, 2000.

Putaud, J. P., Van Dingenen, R., Dell'Acqua, A., Raes, F., Matta, E., Decesari, S., Facchini, M. C., and Fuzzi, S.: Size-segregated aerosol mass closure and chemical composition in Monte $\mathrm{Ci}$ mone (I) during MINATROC, Atmos. Chem. Phys., 4, 889-902, 2004 , http://www.atmos-chem-phys.net/4/889/2004/.

Quaas, J., Boucher, O., and Bréon, F.-M.: Aerosol indirect effects in POLDER satellite data and the Laboratoire de Météorologie Dynamique-Zoom (LMDZ) general circulation model, J. Geophys. Res.-Atmos., 109, D08205, doi:10.1029/2003JD004317, 2004.

Quinn, P. K., Bates, T. S., Miller, T. L., Coffman, D. J., Johnson, J. E., Harris, J. M., Ogren, J. A., Forbes, G., Anderson, T. L., Covert, D. S., and Rood, M. J.: Surface submicron aerosol chemical composition: What fraction is not sulfate?, J. Geophys. Res.Atmos., 105, 6785-6805, 2000.

Rader, D. J. and McMurry, P. H.: Application of the Tandem Differential Mobility Analyzer to Studies of Droplet Growth or Evaporation, J. Aerosol Sci., 17, 771-787, 1986.

Ramanathan, V.: Indian Ocean Experiment: An integrated analysis of the climate forcing and effects of the great Indo-Asian haze, J. Geophys. Res.-Atmos., 106, 28 371-28 398, 2001.

Ramaswamy, V., Boucher, O., Haigh, J., Hauglustaine, D., Haywood, J., Myhre, G., Nakajima, T., Shi, G. Y., and Solomon, S.: Climate Change 2001: The Scientific Basis. Contribution of working group I to the Third Assessment Report of the Intergovernmental Panel on Climate Change, 2001.

Randles, C. A., Russell, L. M., and Ramaswamy, V.: Hygroscopic and optical properties of organic sea salt aerosol and consequences for climate forcing, Geophys. Res. Lett., 31, L16 108, doi:10.1029/2004GL020628, 2004.

Raymond, T. M. and Pandis, S. N.: Cloud activation of singlecomponent organic aerosol particles, J. Geophys. Res.-Atmos., 107, 4787, doi:10.1029/2002JD002159, 2002.

Raymond, T. M. and Pandis, S. N.: Formation of cloud droplets by multicomponent organic particles, J. Geophys. Res.-Atmos., 108, 4469, doi:10.1029/2003JD003503, 2003.

Rissler, J., Swietlicki, E., Zhou, J., Roberts, G., Andreae, M. O., Gatti, L. V., and Artaxo, P.: Physical properties of the submicrometer aerosol over the Amazon rain forest during the wetto-dry season transition - comparison of modeled and measured CCN concentrations, Atmos. Chem. Phys., 4, 2119-2143, 2004, http://www.atmos-chem-phys.net/4/2119/2004/.

Rissman, T. A., Nenes, A., and Seinfeld, J. H.: Chemical amplification (or dampening) of the Twomey effect: Conditions derived from droplet activation theory, J. Atmos. Sci., 61, 919-930, 2004.

Roberts, G. C. and Nenes, A.: A continuous-flow streamwise thermal-gradient $\mathrm{CCN}$ chamber for atmospheric measurements, Aerosol Sci. Technol., 39, 206-221, 2005.

Roberts, G. C., Artaxo, P., and Andreae, M. O.: The chemistry and role of cloud condensation nuclei in the Amazon Basin, J. Aerosol Sci., 31, S62-S63, 2000.

Roberts, G. C., Andreae, M. O., Zhou, J., and Artaxo, P.: Cloud condensation nuclei in the Amazon basin: "marine conditions 
over a continent?", Geophys. Res. Lett., 28, 2807-2810, 2001.

Roberts, G. C., Artaxo, P., Zhou, J. C., Swietlicki, E., and Andreae, M. O.: Sensitivity of CCN spectra on chemical and physical properties of aerosol: A case study from the Amazon Basin, J. Geophys. Res.-Atmos., 107, 8070-8088, 2002.

Rogers, R. R. and Yau, M. K.: A short course in cloud physics, vol. 113, International Series in Natural Philosophy, ButterworthHeinemann, third edn., 1989.

Romakkaniemi, S., Kokkola, H., and Laaksonen, A.: Parameterization of the nitric acid effect on CCN activation, Atmos. Chem. Phys., 5, 879-885, 2005,

http://www.atmos-chem-phys.net/5/879/2005/.

Rood, M. J. and Williams, A. L.: Comments on "Influence of soluble surfactant properties on the activation of aerosol particles containing inorganic solute" - Reply, J. Atmos. Sci., 58, 14681473, 2001.

Rotstayn, L. D. and Penner, J. E.: Indirect aerosol forcing, quasi forcing, and climate response, J. Climate, 14, 2960-2975, 2001.

Rupakheti, M. W., Leaitch, R., Lohmann, U., Hayden, K., Brickell, P., Lu, G., Li, S. M., Toom-Sauntry, D., Bottenheim, J. W., Brook, J. R., Vet, R., Jayne, J. T. and Worsnop, D. R.: An intensive study of the size and composition of submicron atmospheric aerosols at a rural site in Ontario, Canada, Aerosol Sci. Technol., 39, 722-736, 2005.

Russell, L. M.: Aerosol organic-mass-to-organic-carbon ratio measurements, Abstracts of Papers of the American Chemical Society, 225, U817-U818, part 1, 2003.

Saathoff, H., Naumann, K. H., Schnaiter, M., Schock, W., Mohler, O., Schurath, U., Weingartner, E., Gysel, M., and Baltensperger, U.: Coating of soot and (NH4)(2)SO4 particles by ozonolysis products of alpha-pinene, J. Aerosol Sci., 34, 1297-1321, 2003.

Santarpia, J. L., Li, R. J., and Collins, D. R.: Direct measurement of the hydration state of ambient aerosol populations, J. Geophys. Res., 109, D18209, doi:10.1029/2004JD004653. 2004

Sardar, S. B., Fine, P. M., and Sioutas, C.: Seasonal and spatial variability of the size-resolved chemical composition of particulate matter (PM10) in the Los Angeles Basin, J. Geophys. Res.Atmos., 110, D07S08, doi:10.1029/2004JD004627, 2005.

Saxena, P. and Hildemann, L. M.: Water-soluble organics in atmospheric particles: A critical review of the literature and application of thermodynamics to identify candidate compounds, J. Atmos. Chem., 24, 57-109, 1996.

Saxena, P., Hildemann, L. M., McMurry, P. H., and Seinfeld, J. H.: Organics Alter Hygroscopic Behavior of Atmospheric Particles, J. Geophys. Res.-Atmos., 100, 18 755-18 770, 1995.

Schkolnik, G., Falkovich, A. H., Rudich, Y., Maenhaut, W., and Artaxo, P.: New analytical method for the determination of levoglucosan, polyhydroxy compounds, and 2-methylerythritol and its application to smoke and rainwater samples, Environ. Sci. Technol., 39, 2744-2752, 2005.

Schneider, J., Borrmann, S., Wollny, A. G., Blasner, M., Mihalopoulos, N., Oikonomou, K., Sciare, J., Teller, A., Levin, Z., and Worsnop, D. R.: Online mass spectrometric aerosol measurements during the MINOS campaign (Crete, August 2001), Atmos. Chem. Phys., 4, 65-80, 2004, http://www.atmos-chem-phys.net/4/65/2004/.

Schulz, M., Balkanski, Y. J., Guelle, W., and Dulac, F.: Role of aerosol size distribution and source location in a threedimensional simulation of a Saharan dust episode tested against satellite-derived optical thickness, J. Geophys. Res.-Atmos., 103, 10 579-10 592, 1998.

Schwartz, S. E.: Uncertainty requirements in radiative forcing of climate change, J. Air \& Waste Manage. Assoc., 54, 1351-1359, 2004.

Seidl, W.: Model for a surface film of fatty acids on rain water and aerosol particles, Atmos. Environ., 34, 4917-4932, 2000.

Seidl, W. and Hänel, G.: Surface-active substances on rainwater and atmospheric particles, Pure Appl. Geophys., 121, 1077-1093, 1983.

Seinfeld, J. H. and Pandis, S. N.: Atmospheric chemistry and physics: from air pollution to climate change, John Wiley \& Sons, New York; Chichester, air pollution to climate, 1998.

Sellegri, K., Laj, P., Peron, F., Dupuy, R., Legrand, M., Preunkert, S., Putaud, J. P., Cachier, H., and Ghermandi, G.: Mass balance of free tropospheric aerosol at the Puy de D(o)overcapme (France) in winter, J. Geophys. Res.-Atmos., 108, 4333, doi:10.1029/2002JD002747, 2003.

Shantz, N. C., Leaitch, W. R., and Caffrey, P. F.: Effect of organics of low solubility on the growth rate of cloud droplets, J. Geophys. Res.-Atmos., 108, 4168, doi:10.1029/2002JD002540, 2003.

Shaw, R. A. and Lamb, D.: Experimental determination of the thermal accommodation and condensation coefficients of water, J. Chem. Phys., 111, 10 659-10 663, 1999.

Shulman, M. L., Jacobson, M. C., Carlson, R. J., Synovec, R. E., and Young, T. E.: Dissolution behavior and surface tension effects of organic compounds in nucleating cloud droplets, Geophys. Res. Lett., 23, 277-280, 1996.

Silva Dias, M. A. F., Rutledge, S., Kabat, P., Silva Dias, P. L., Nobre, C., Fisch, G., Dolman, A. J., Zipser, E., Garstang, M., Manzi, A., Fuentes, J. D., Rocha, H., Marengo, J., Plana-Fattori, A., Alvalá, L. S. R., Andreae, M. O., Artaxo, P., Gielow, R., and Gatti, L. V.: Clouds and rain processes in a biosphere atmosphere interaction context in the Amazon Region, J. Geophys. Res.Atmos., 107, 8072-8092, doi:10.1029/2001JD000335, 2002.

Silva Dias, M. A. F., Artaxo, P., and Andreae, M. O.: Aerosols impact clouds in the Amazon Basin, GEWEX Newsletter, 14, 4-6, 2004.

Sinnarwalla, A. M. and Alofs, D. J.: A cloud nucleus counter with long available growth time, J. Appl. Meteorol., 12, 831-835, 1972.

Slauenwhite, D. E. and Johnson, B. D.: Bubble shattering: Differences in bubble formation in fresh water and seawater, J. Geophys. Res.-O, 104, 3265-3275, 1999.

Snider, J. R. and Brenguier, J. L.: Cloud condensation nuclei and cloud droplet measurements during ACE-2, Tellus B, 52, 828842, 2000.

Snider, J. R., Guibert, S., Brenguier, J. L., and Putaud, J. P.: Aerosol activation in marine stratocumulus clouds: 2. Köhler and parcel theory closure studies, J. Geophys. Res.-Atmos., 108, 8629, doi:10.1029/2002JD002 692, 2003.

Sorjamaa, R., Svenningsson, B., Raatikainen, T., Henning, S., Bilde, M., and Laaksonen, A.: The role of surfactants in Kohler theory reconsidered, Atmos. Chem. Phys., 4, 2107-2117, 2004, http://www.atmos-chem-phys.net/4/2107/2004/.

Spracklen, D. V., Pringle, K. J., Carslaw, K. S., Chipperfield, M. P., and Mann, G. W.: A global off-line model of size-resolved aerosol microphysics: I. Model development and prediction of aerosol properties, Atmos. Chem. Phys., 5, 2227-2252, 2005, 
http://www.atmos-chem-phys.net/5/2227/2005/.

Squires, P. and Twomey, S.: A Comparison of Cloud Nucleus Measurements over Central North America and Caribbean Sea, J. Atmos. Sci., 23, 401-404, 1966.

Stier, P., Feichter, J., Kinne, S., Kloster, S., Vignati, E., Wilson, J., Ganzeveld, L., Tegen, I., Werner, M., Balkanski, Y., Schulz, M., and Boucher, O.: The aerosol-climate model ECHAM5-HAM, Atmos. Chem. Phys., 5, 1125-1156, 2005, http://www.atmos-chem-phys.net/5/1125/2005/.

Stolzenburg, M. R. and McMurry, P. H.: TDMAFIT user's manual, Tech. rep., University of Minnesota, Department of Mechanical Engineering, Particle Technology Laboratory, Minneapolis, 1988.

Stratmann, F., Orsini, D., and Kauffeldt, T.: Inversion algorithm for TDMA measurements, J. Aeros. Sci., 28, S701-S702, 1997.

Stratmann, F., Kiselev, A., Wurzler, S., Wendisch, M., Heintzenberg, J., Charlson, R. J., Diehl, K., Wex, H., and Schmidt, S.: Laboratory studies and numerical simulations of cloud droplet formation under realistic supersaturation conditions, J. Atmos. Ocean. Techn., 21, 876-887, 2004.

Sullivan, A. P., Weber, R. J., Clements, A. L., Turner, J. R., Bae, M. S., and Schauer, J. J.: A method for on-line measurement of water-soluble organic carbon in ambient aerosol particles: Results from an urban site, Geophys. Res. Lett., 31, L13 105, doi:10.1029/2004GL019681, 2004.

Svenningson, B., Hansson, H.-C., Wiedensohler, A., Ogren, J., Noone, K. and Hallberg, A.: Hygroscopic growth of aerosol particles in the Po Valley, Tellus B, 44, 556-569, 1992.

Svenningsson, B., Hansson, H. C., Wiedensohler, A., Noone, K., Ogren, J., Hallberg, A., and Colvile, R.: Hygroscopic growth of aerosol-particles and its influence on nucleation scavenging incloud - experimental results from Kleiner-Feldberg, J. Atmos. Chem., 19, 129-152, 1994.

Svenningsson, B., Hansson, H. C., Martinsson, B., Wiedensohler, A., Swietlicki, E., Cederfelt, S. I., Wendisch, M., Bower, K. N., Choularton, T. W., and Colvile, R. N.: Cloud droplet nucleation scavenging in relation to the size and hygroscopic behaviour of aerosol particles, Atmos. Environ., 31, 2463-2475, 1997.

Swietlicki, E., Zhou, J. C., Berg, O. H., Martinsson, B. G., Frank, G., Cederfelt, S. I., Dusek, U., Berner, A., Birmili, W., Wiedensohler, A., Yuskiewicz, B., and Bower, K. N.: A closure study of sub-micrometer aerosol particle hygroscopic behaviour, Atmos. Res., 50, 205-240, 1999.

Swietlicki, E., Zhou, J. C., Covert, D. S., Hameri, K., Busch, B., Vakeva, M., Dusek, U., Berg, O. H., Wiedensohler, A., Aalto, P., Makela, J., Martinsson, B. G., Papaspiropoulos, G., Mentes, B., Frank, G., and Stratmann, F.: Hygroscopic properties of aerosol particles in the northeastern Atlantic during ACE-2, Tellus B, 52, 201-227, 2000.

Takemura, T., Nozawa, T., Emori, S., Nakajima, T. Y., and Nakajima, T.: Simulation of climate response to aerosol direct and indirect effects with aerosol transport-radiation model, J. Geophys. Res., 110, D02202, doi:10.1029/2004JD00502, 2005.

Tegen, I. and Fung, I.: Modeling of Mineral Dust in the Atmosphere - Sources, Transport, and Optical-Thickness, J. Geophys. Res.Atmos., 99, 22 897-22 914, 1994.

Temesi, D., Molnar, A., Meszaros, E., Feczko, T., Gelencser, A., Kiss, G., and Krivacsy, Z.: Size resolved chemical mass balance of aerosol particles over rural Hungary, Atmos. Environ.,
35, 4347-4355, 2001.

Tervahattu, H., Juhanoja, J., Vaida, V., Tuck, A. F., Niemi, J. V., Kupiainen, K., Kulmala, M., and Vehkamaki, H.: Fatty acids on continental sulfate aerosol particles, J. Geophys. Res.-Atmos., 110, D06 207, doi:10.1029/2004JD005400, 2005.

Tolocka, M. P., Jang, M., Ginter, J. M., Cox, F. J., Kamens, R. M., and Johnston, M. V.: Formation of oligomers in secondary organic aerosol, Environ. Sci. Technol., 38, 1428-1434, 2004.

Topping, D., Coe, H., McFiggans, G., Burgess, R., Allan, J., Alfarra, M. R., Bower, K., Choularton, T. W., Decesari, S., and Facchini, M. C.: Aerosol chemical characteristics from sampling conducted on the Island of Jeju, Korea during ACE Asia, Atmos. Environ., 38, 2111-2123, 2004.

Topping, D. O., McFiggans, G. B., and Coe, H.: A curved multicomponent aerosol hygroscopicity model framework: Part 1 - Inorganic compounds, Atmos. Chem. Phys., 5, 1205-1222, 2005a.

Topping, D. O., McFiggans, G. B., and Coe, H.: A curved multicomponent aerosol hygroscopicity model framework: Part 2 Including organic compounds, Atmos. Chem. Phys., 5, 12231242, 2005 b.

Turpin, B. J. and Lim, H. J.: Species contributions to PM2.5 mass concentrations: Revisiting common assumptions for estimating organic mass, Aerosol Sci. Technol., 35, 602-610, 2001.

Twomey, S.: The nuclei of natural cloud formation, Part II: The supersaturation in natural clouds and the variation of cloud droplet concentration, Geofis. Pura Appl., 43, 243-249, 1959.

Twomey, S.: Pollution and Planetary Albedo, Atmos. Environ., 8, 1251-1256, 1974.

Van Dingenen, R., Raes, F., Putaud, J. P., Baltensperger, U., Charron, A., Facchini, M. C., Decesari, S., Fuzzi, S., Gehrig, R., Hansson, H. C., Harrison, R. M., Huglin, C., Jones, A. M., Laj, P., Lorbeer, G., Maenhaut, W., Palmgren, F., Querol, X., Rodriguez, S., Schneider, J., ten Brink, H., Tunved, P., Torseth, K., Wehner, B., Weingartner, E., Wiedensohler, A., and Wahlin, P.: A European aerosol phenomenology-1: physical characteristics of particulate matter at kerbside, urban, rural and background sites in Europe, Atmos. Environ., 38, 2561-2577, 2004.

Van Dingenen, R., Raes, F., Putaud, J. P., Virkkula, A., and Mangoni, M.: Processes determining the relationship between aerosol number and non-sea-salt sulfate mass concentrations in the clean and perturbed marine boundary layer, J. Geophys. Res., 104, 8027-8038, 1999.

VanReken, T. M., Rissman, T. A., Roberts, G. C., Varutbangkul, V., Jonsson, H. H., Flagan, R. C., and Seinfeld, J. H.: Toward aerosol/cloud condensation nuclei $(\mathrm{CCN})$ closure during CRYSTAL-FACE, J. Geophys. Res.-Atmos., 108, 4633, doi:10.1029/2003JD003582, 2003.

Varga, B., Kiss, G., Ganszky, I., Gelencser, A., and Krivacsy, Z.: Isolation of water-soluble organic matter from atmospheric aerosol, Talanta, 55, 561-572, 2001.

Virkkula, A., Van Dingenen, R., Raes, F., and Hjorth, J.: Hygroscopic properties of aerosol formed by oxidation of limonene, alpha -pinene, and beta -pinene, J. Geophys. Res., 104, 35693579, 1999.

Vlasenko, A., Sjogren, S., Weingartner, E., Gaggeler, H. W., and Ammann, M.: Generation of submicron Arizona test dust aerosol: Chemical and hygroscopic properties, Aerosol Sci. Technol., 39, 452-460, 2005. 
Voutilainen, A., Stratmann, F., and Kaipio, J. P.: A nonhomogeneous regularization method for the estimation of narrow aerosol size distributions, J. Aeros. Sci., 31, 1433-1445, 2000.

Warner, J.: A reduction of rain associated with smoke from sugarcane fires-An inadvertent weather modification, J. Appl. Meteorol., 7, 247-251, 1968.

Weingartner, E., Baltensperger, U., and Burtscher, H.: Growth and structural change of combustion aerosols at high relative humidity, Environ. Sci. Technol., 29, 12, 2982-2986, 1995.

Weingartner, E., Burtscher, H., Baltensperger, U.: Hygroscopic properties of carbon and diesel soot particles, Atmos. Environ., 31, 15, 2311-2327, 1997.

Weingartner, E., Nyeki, S., and Baltensperger, U.: Seasonal and diurnal variation of aerosol size distributions $(10<\mathrm{D}<750 \mathrm{~nm})$ at a high-alpine site (Jungfraujoch $3580 \mathrm{~m}$ a.s.l.), J. Geophys. Res., 104, 26 809-26820, 1999.

Weingartner, E., Henning, S., Gysel, M., Bukowiecki, N., and Baltensperger, U.: Hygroscopicity of aerosol particles at low temperatures, J. Aerosol. Sci., 32, S977-S978, 2001.

Weingartner, E., Gysel, M., and Baltensperger, U.: Hygroscopicity of aerosol particles at low temperatures. 1. New low-temperature H-TDMA instrument: Setup and first applications, Environ. Sci. Technol., 36, 55-62, 2002.

Weingartner, E., Sjögren, S., Cozic, J., Verheggen, B., Baltensperger, U., Alfarra, M. R., Bower, K. N., Flynn, M. J., Gysel, M., and Coe, H.: Hygroscopic properties and chemical composition of aerosol particles at the high alpine site Jungfraujoch, J. Aerosol Sci., 35, S135-S136, 2004.

Wieland, W.: Die Wasserdampfkondensation an natülichem Aerosol bei geringen übersättigungen, Z. Angew. Math. Phys., 7, 428-460, 1956.

Whitby, K. T.: The physical characteristics of sulphur aerosols, Atmos. Environ., 12, 135-159, 1978.

Williams, K. D., Jones, A., Roberts, D. L., Senior, C. A., and Woodage, M. J.: The response of the climate system to the indirect effects of anthropogenic sulfate aerosol, Climate Dynamics, 17, 845-856, 2001.

Wilson, J., Cuvelier, C., and Raes, F.: The response of the climate system to the indirect effects of anthropogenic sulfate aerosol, A modelling study of global mixed aerosol fields, J. Geophys. Res., 106(D24), 34 081-34 108, doi:10.1029/2000JD000198, 2001.

Winkler, P. M., Vrtala, A., Wagner, P., Kulmala, M., Lehtinen, K. E. J., and Vesala, T.: Mass and thermal accommodation during gas-liquid condensation of water, Phys. Rev. Lett., 93, 075701, doi:10.1103/PhysRevLett.93.075701, 2004.

Wise, M. E, Surratt, J. D, Curtis, D. B, Shilling, J. E, and Tolbert, M. A.: Hygroscopic growth of ammonium sulfate/dicarboxylic acids, J. Geophys. Res., 108(D20), 4638, doi:10.1029/2003JD003775, 2003.

Xiao, H. Y. and Liu, C. Q.: Chemical characteristics of watersoluble components in TSP over Guiyang, SW China, 2003, Atmos. Environ., 38, 6297-6306, 2004.

Xiong, J. Q., Zhong, M. H., Fang, C. P., Chen, L. C., and Lippmann, M.: Influence of organic films on the hygroscopicity of ultrafine sulfuric acid aerosol, Environ. Sci. Technol., 32, 35363541, 1998.

Xue, H. W. and Feingold, G.: A modeling study of the effect of nitric acid on cloud properties, J. Geophys. Res.-Atmos., 109, D18 204, doi:10.1029/2004JD004750, 2004.
Yu, J. Z., Yang, H., Zhang, H. Y., and Lau, A. K. H.: Size distributions of water-soluble organic carbon in ambient aerosols and its size-resolved thermal characteristics, Atmos. Environ., 38, 1061-1071, 2004.

Yum, S. S., Hudson, J. G., and Xie, Y. H.: Comparisons of cloud microphysics with cloud condensation nuclei spectra over the summertime Southern Ocean, J. Geophys. Res., 103, 16 625-16 636, 1998.

Zappoli, S., Andracchio, A., Fuzzi, S., Facchini, M. C., Gelencser, A., Kiss, G., Krivacsy, Z., Molnar, A., Meszaros, E., Hansson, H. C., Rosman, K., and Zebuhr, Y.: Inorganic, organic and macromolecular components of fine aerosol in different areas of Europe in relation to their water solubility, Atmos. Environ., 33, 2733-2743, 1999.

Zhang, Q. and Anastasio, C.: Chemistry of fog waters in California's Central Valley - Part 3: concentrations and speciation of organic and inorganic nitrogen, Atmos. Environ., 35(32), 56295643, 2001.

Zhang, Q. and Anastasio, C.: Free and combined amino compounds in atmospheric fine particles (PM2.5) and fog waters from Northern California, Atmos. Environ., 37(16), 2247-2258, 2003.

Zhang, X. Q., McMurry, P. H., Hering, S. V., and Casuccio, G. S.: Mixing Characteristics and Water-Content of Submicron Aerosols Measured in Los-Angeles and at the Grand-Canyon, Atmos. Environ. Part a - General Topics, 27, 1593-1607, 1993.

Zhou, J. C., Swietlicki, E., Berg, O. H., Aalto, P. P., Hameri, K., Nilsson, E. D., and Leck, C.: Hygroscopic properties of aerosol particles over the central Arctic Ocean during summer, J. Geophys. Res.-Atmos., 106, 32 111-32 123, 2001.

Zhang, Q., Anastasio, C., and Jimemez-Cruz, M.: Watersoluble organic nitrogen in atmospheric fine particles (PM2.5) from northern California, J. Geophys. Res.-Atmos., 107(D11), doi:10.1029/2001JD000870, 2002a.

Zhang, Y., Easter, R. C., Ghan, S. J., and Abdul-Razzak, H.: Impact of aerosol size representation on modeling aerosolcloud interactions, J. Geophys. Res.-Atmos., 107, 4558, doi:10.1029/2001JD001549, 2002b.

Zhang, Q., Stanier, C. O., Canagaratna, M. R., Jayne, J. T., Pandis, S. N., Worsnop, D. R., and Jimenez, J. L.: Insights into the Chemistry of Nucleation Bursts and New Particle Growth Events in Pittsburgh Based on Aerosol Mass Spectrometry, Environ. Sci. Technol., 38, 4797-4809, 2004.

Zhang, Q., Alfarra, M. R., Worsnop, D. R., Allan, J. D., Coe, H., Canagaratna, M. R., and Jimenez, J. L.: Deconvolution and quantification of hydrocarbon-like and oxygenated organic aerosols based on aerosol mass spectrometry, Environ. Sci. Technol., 39, 4938-4952, doi:10.1021/es0485681, 2005.

Zhang, Q., Worsnop, D. R., Canagaratna, M. R., Jayne, J. T., and Jimenez, J. L.: Hydrocarbon-like and Oxygenated Organic Aerosols in Pittsburgh: Insights into Sources and Processes of Organic Aerosols, Atmos. Chem. Phys., 5, 3289-3311, 2005, http://www.atmos-chem-phys.net/5/3289/2005/.

Zhou, J., Swietlicki, E., Hansson, H. C., and Artaxo P.: Submicrometer aerosol particle size distribution and hygroscopic growth measured in the Amazon rain forest during the wet season, J. Geophys. Res., 107(D20), 8055, doi:10.1029/2000JD000203, 2002. 